\title{
Determination and use of scatter correction factors of megavoltage photon beams
}

Measurement and use of collimator and phantom scatter correction factors of arbitrarily shaped fields with a symmetrical collimator setting

NEDERLANDSE COMMISSIE VOOR STRALINGSDOSIMETRIE

Report 12 of the Netherlands Commission on Radiation Dosimetry

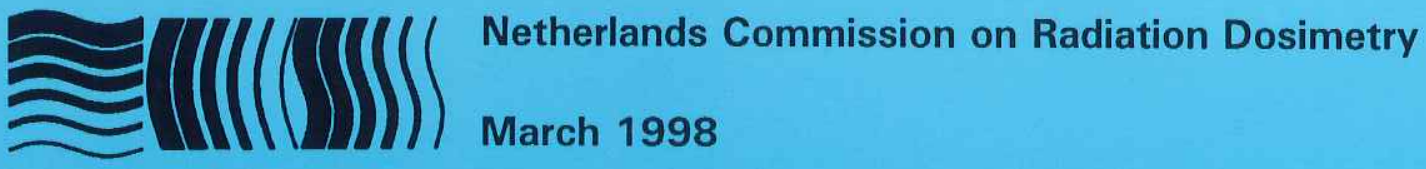




\section{Disclaimer regarding NCS reports}

The NCS frequently publishes reports for fellow professionals in which recommendations are given for various quality control procedures or otherwise. The members of the NCS board and the members of the concerning subcommittee do not claim any authority exceeding that of their professional expertise. Responsibility on how the NCS recommendations are implemented lies with the user, taking into account the practice in his/her institution.

Note:

This report is partially superseded by NCS report 15, Quality assurance of 3-D treatment planning systems for external photon and electron beams.

Please note that a new version should occur before 2020. 


\section{Determination and use of scatter correction factors of megavoltage photon beams}

Measurement and use of collimator and phantom scatter correction factors of arbitrarily shaped fields with a symmetrical collimator setting

NEDERLANDSE COMMISSIE VOOR STRALINGSDOSIMETRIE

Report 12 of the Netherlands Commission on Radiation Dosimetry

Authors:

J.J.M. van Gasteren

S. Heukelom

H.N. Jager

B.J. Mijnheer

R. van der Laarse

H.J. van Kleffens

J.L.M. Venselaar

C.F. Westermann

Netherlands Commission on Radiation Dosimetry

March 1998 


\section{Preface}

The Nederlandse Commissie voor Stralingsdosimetrie (NCS, Netherlands Commission on Radiation Dosimetry) was officially established on 3 September 1982 with the aim of promoting the appropriate use of dosimetry of ionizing radiation both for scientific research and practical applications. The NCS is chaired by a board of scientists, installed upon the suggestion of the supporting societies, including the Nederlandse Vereniging voor Radiotherapie en Oncologie (Netherlands Society for Radiotherapy and Oncology), the Nederlandse Vereniging voor Klinische Fysica (Netherlands Society for Clinical Physics), the Nederlandse Vereniging voor Radiobiologie (Netherlands Society for Radiobiology), the Nederlandse Vereniging voor Stralingshygiëne (Netherlands Society for Radiological Protection), the Nederlandse Vereniging van Radiologisch Laboranten (Netherlands Society of Radiographers and Radiological Technologists), and the Ministry of Health, Welfare and Sports.

To pursue its aims, the NCS accomplishes the following tasks: participation in dosimetry standardisation and promotion of dosimetry intercomparisons, drafting of dosimetry protocols, collection and evaluation of physical data related to dosimetry. Furthermore, the commission shall maintain or establish links with national and international organisations concerned with ionizing radiation and promulgate information on new developments in the field of radiation dosimetry.

Current members of the board of the NCS:

J.J. Broerse, chairman W. de Vries, secretary

J. Zoetelief, treasurer A.J.J. Bos W.C.A.M. Buijs R.B. Keus J.L.M. Venselaar F.W. Wittkämper D. Zweers 


\section{Determination and use of scatter correction factors of megavoltage photon beams}

Measurement and use of collimator and phantom scatter correction factors of arbitrarily shaped fields with a symmetrical collimator setting

Prepared by the Task Group on Dose Calculations for External Beam Radiotherapy of the Netherlands Society on Radiation Dosimetry (NCS).

Members of the Task Group:

J.J.M. van Gasteren

S. Heukelom H.N. Jager

B.J. Mijnheer

R. van der Laarse

H.J. van Kleffens

J.L.M. Venselaar

C.F. Westermann 


\section{Contents}

Preface

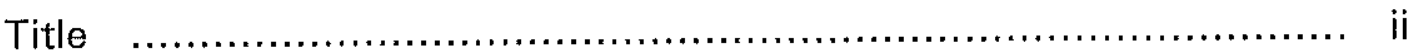

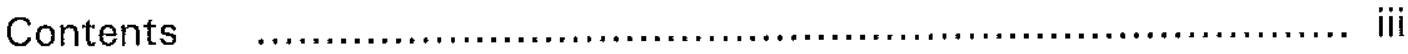

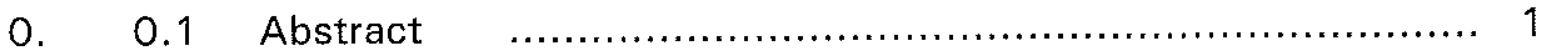

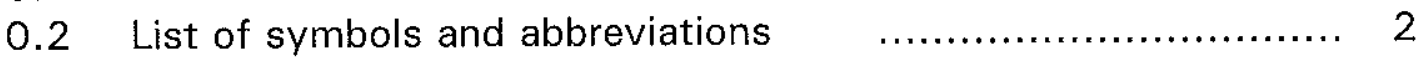

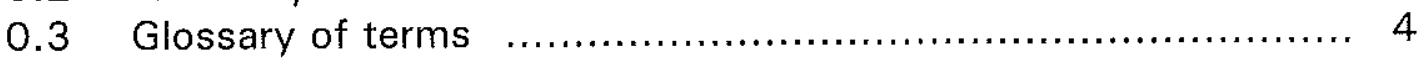

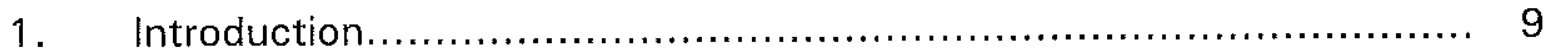

2. Collimator and phantom scatter correction factors for square fields .. 13

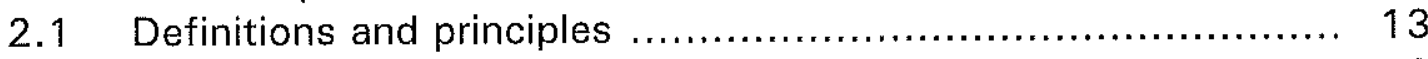

2.2 Calibration and reference irradiation conditions $\ldots \ldots \ldots \ldots \ldots . .18$

2.3 Calculation of the dose under non-reference conditions $\ldots \ldots . .20$

2.4 Calculation of treatment time and monitor units in the fixed SSD

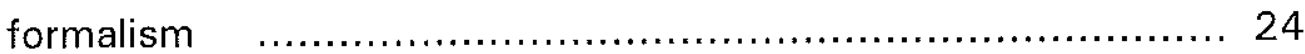

2.5 Calculation of monitor units in the isocentric formalism $\ldots \ldots .25$

3. Scatter correction factors for non-square fields, fields with wedges,

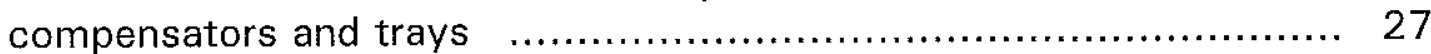

3.1 Rectangular and irregularly shaped fields $\ldots \ldots \ldots \ldots \ldots \ldots \ldots, 27$

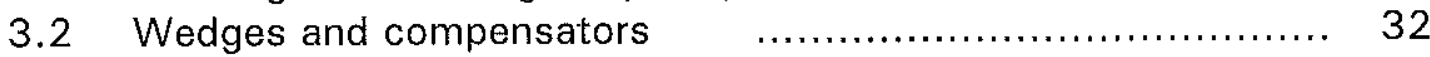

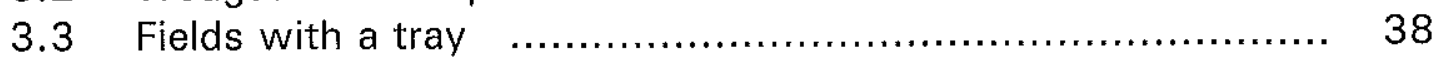

3.4 Recommendations with respect to monitor unit calculations... 41

4. Experimental determination of scatter correction factors $\quad . . \ldots \ldots \ldots . . .43$

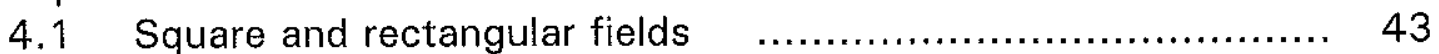

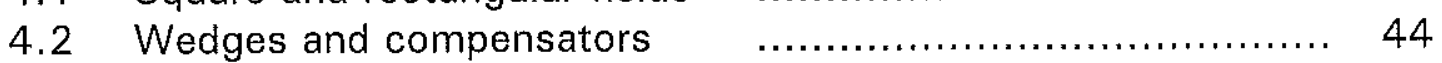

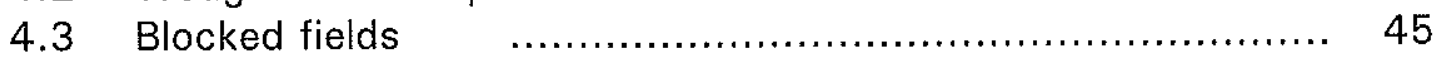

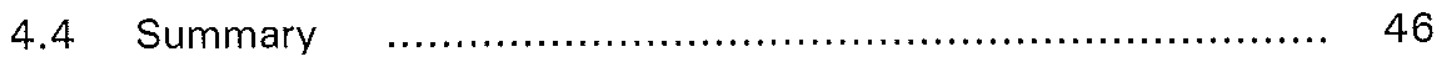

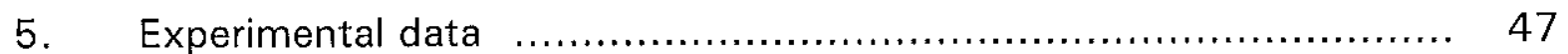

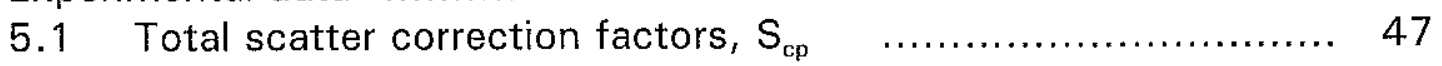

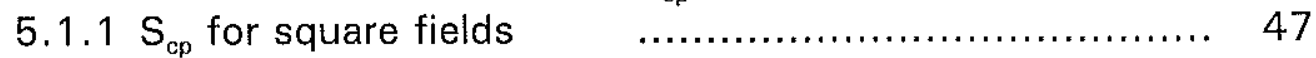

5.2 Collimator scatter correction factors, $S_{c} \quad \ldots \ldots \ldots \ldots \ldots \ldots \ldots . \ldots 47$

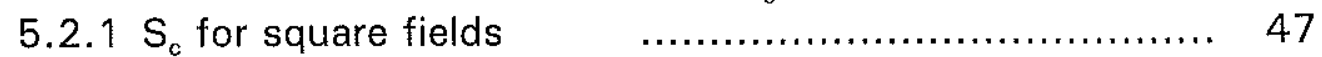

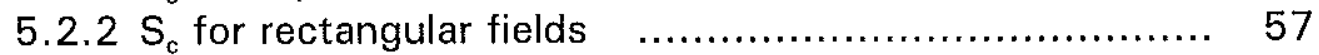

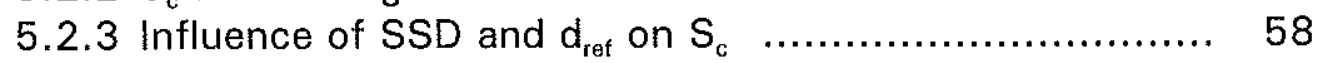

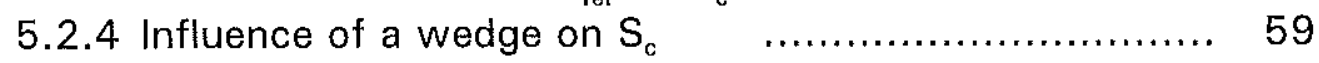


5.3 Phantom scatter correction factors, $S_{p} \ldots \ldots \ldots \ldots \ldots \ldots \ldots \ldots \ldots, 60$

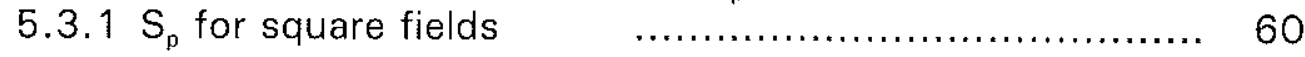

5.3.2 $S_{p}$ for rectangular fields $\quad \ldots \ldots \ldots \ldots \ldots \ldots \ldots \ldots \ldots \ldots \ldots, 62$

5.3.3 Comparison of measured and calculated $S_{p}$ values for rectangular fields

6. Recommendations

7. Acknowledgements

8. Appendices

8.1 Definition of scatter correction factors $\quad \ldots \ldots \ldots \ldots \ldots \ldots \ldots .6 .69$

8.2 Scatter correction factors at other reference depths $\ldots . .71$

8.3 Relations between the quantities in the fixed SSD and the

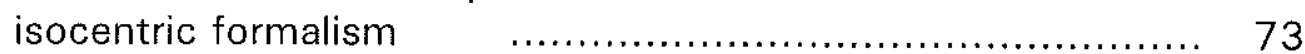

8.4 $S_{p}$ and PDD (and RDD) for arbitrarily shaped fields ............ 77

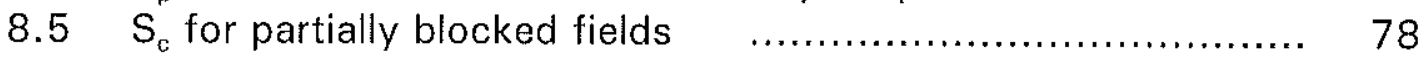

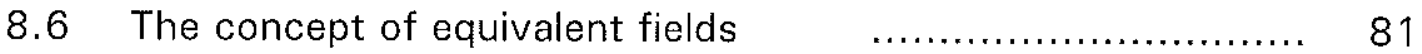

8.7 Parametrization of scatter correction factors $\quad \ldots \ldots \ldots \ldots . . \quad 84$

8.7.1 Parametrization of phantom scatter correction factors . 84

8.7.2 Parametrization of collimator scatter correction factors . 85

8.8 The narrow cylindrical beam-coaxial phantom (mini-phantom). 88

9. References 


\subsection{Abstract}

For each radiation treatment machine, the dose per monitor unit must be known at a reference point in a water-phantom under reference conditions. It varies with field size, due to: (1) changes in radiation scattered from the head of the treatment machine to the reference point and into the monitor chamber and (2) changes in the radiation scattered from the irradiated part of the phantom to the reference point. The field size dependence is usually described by the total scatter correction factor, $\mathrm{S}_{\mathrm{cp}}$. If the field size defined by the collimator setting at the reference source-surface distance (SSD) does not correspond with the field size at the phantom surface, $S_{c p}$ must be separated into two factors, the head or collimator scatter correction factor, $\mathrm{S}_{\mathrm{c}}$, and the phantom scatter correction factor, $S_{p}$. This is the case when a source-surface distance different from the SSD of the reference condition is used, when tissue is missing in the beam, or when shielding blocks are applied. The factor $S_{c}$ describes the influence of the setting of the collimator on the total scatter correction factor; $S_{p}$ describes the influence of the field size at the phantom surface, and thus of the irradiated volume on this correction factor.

In this report, recommendations are given for the measurement of $S_{c p}, S_{c}$ and $S_{p}$ in a photon beam at a reference depth of $10 \mathrm{~cm}$. The reference field is defined as the open field, with a collimator setting yielding a $10 \mathrm{~cm} \times 10 \mathrm{~cm}$ field when the SSD is set equal to the source-axis distance (SAD). For the determination of $S_{c}$, the use of a narrow cylindrical beam-coaxial phantom (the mini-phantom) is recommended. In this way, measurements can be performed in small fields and the disturbance of contaminating electrons, reaching the point of interest from the head of the treatment machine, becomes negligible. Construction details of the mini-phantom are described.

Furthermore, a consistent set of relations is presented for the use of these factors in dose calculations for symmetrically collimated square, rectangular and arbitrarily shaped fields, at an arbitrary SSD. These include blocked and wedged fields. A procedure to use $S_{c}$ and $S_{p}$ data in the calculation of monitor units is presented. Relations are given to calculate the collimator and phantom scatter data for the reference situation from already available data, measured at a nonreference depth and at a non-reference SSD. The influence of asymmetric set-up of the collimating jaws or multi-leaf collimators on the scatter correction factors is, however, not yet considered.

$S_{c p}, S_{c}$ and $S_{p}$ data sets are presented in this report for different types of treatment machines and for a wide range of photon beams, with beam qualities ranging from ${ }^{60} \mathrm{Co}$ to $25 \mathrm{MV}$. $S_{p}$ is shown to be a smooth function of the beam quality if the same reference depth of $10 \mathrm{~cm}$ and an SSD of $100 \mathrm{~cm}$ is chosen for all beam qualities. However, $S_{c}$ is shown to depend on the design of the head of the linear accelerator. 


\subsection{List of symbols and abbreviations}

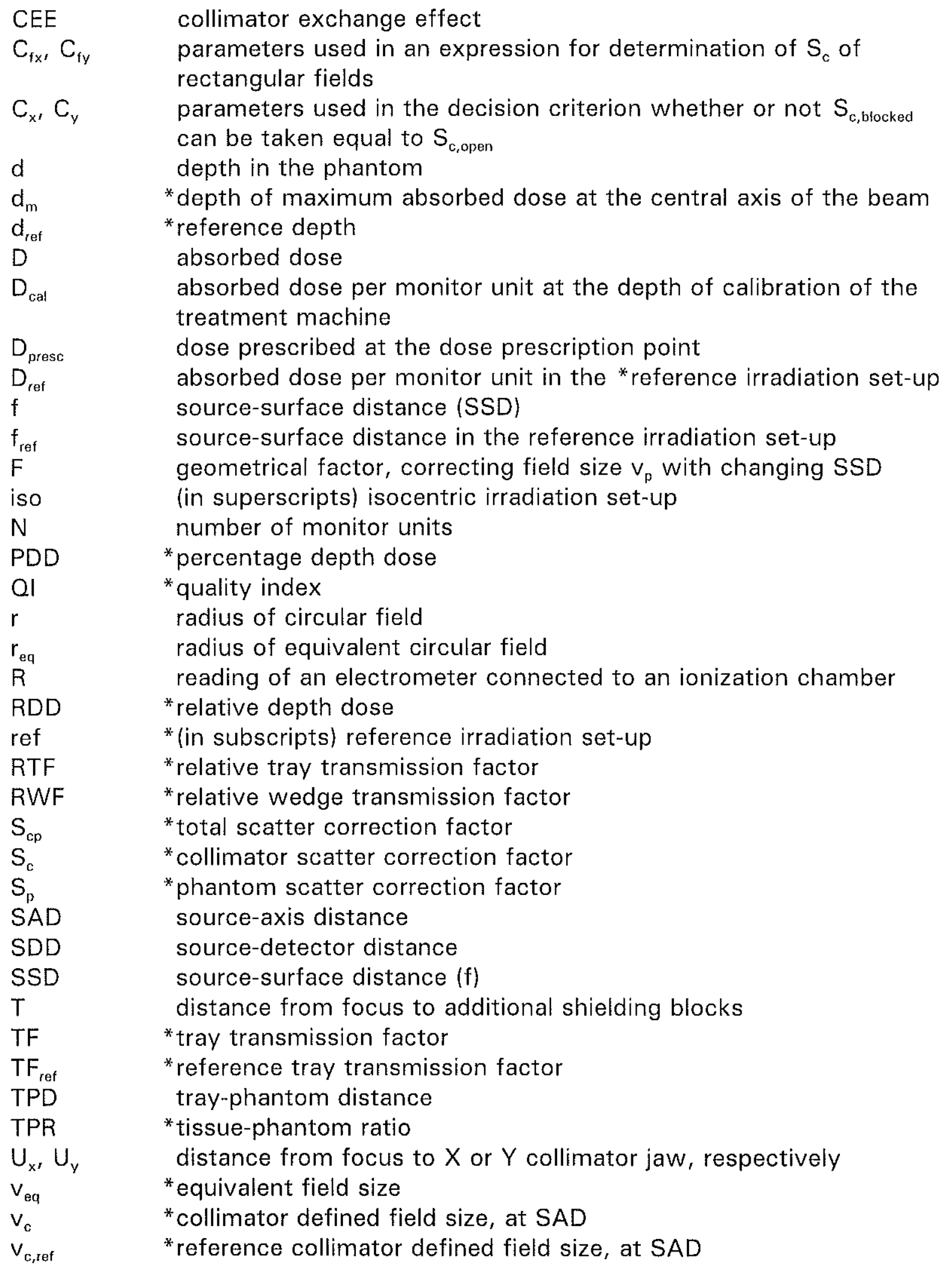


0.

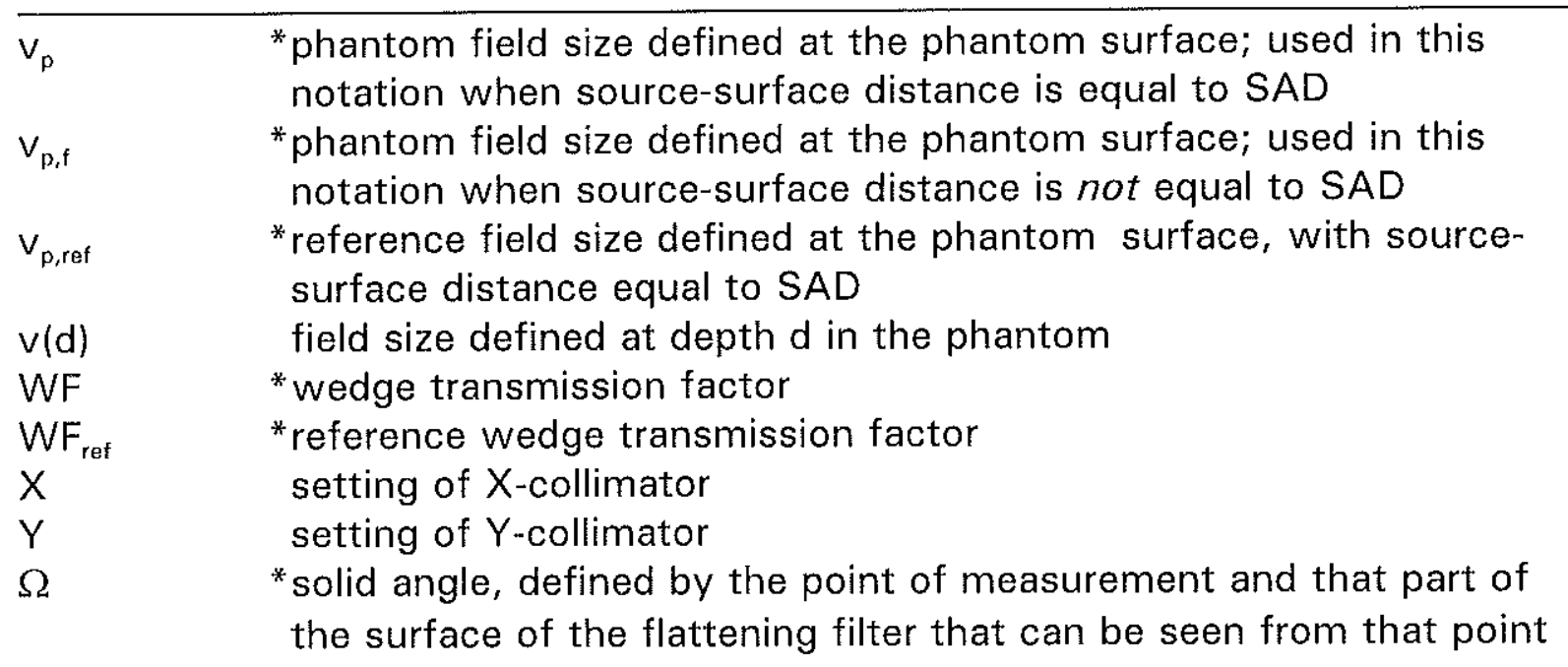

(*) See section 0.3 for a more complete definition. 


\subsection{Glossary of terms}

In this section definitions are given of quantities used in this report as far as they are important for understanding or if they deviate in one or more respects from the definitions given in the 'Glossary of terms' of the British Journal of Radiology Supplement 17, pp 143-147 [8] and Supplement 25, pp 183-188 [9].

\section{collimator scatter correction factor $S_{c}$}

The collimator scatter correction factor $S_{c}$ (also called the head scatter correction factor) is defined as the electrometer reading per monitor unit of an ionization chamber measured in a mini-phantom at the reference depth and the reference source-surface distance for a specified collimator defined field size $v_{c}$, normalized to unity for the reference field size $v_{c, \text { ref }}: S_{c}\left(v_{c}\right)=R\left(v_{c}\right) / R\left(v_{c, \text { ref }}\right)$. This factor describes the relative energy fluence per monitor unit due to photons originating in the head of the treatment machine only.

\section{collimator defined field size $v_{c}$}

The field size $v_{c}$ is determined by the adjustable collimator jaws in the head of the treatment machine. The setting of the jaws defines a field of size $v_{c}$ at $a$ distance from the source equal to $S A D$. In most clinical cases, $v_{c}$ defines the solid angle $\Omega$. In the text, often the term collimator setting is used for $v_{c}$.

\section{depth of maximum dose $d_{m}$}

The depth $d_{m}$ of maximum absorbed dose is the depth along the beam axis at which maximum dose occurs. For a given beam quality this depth will vary with field size and source-surface distance. However, percentage depth doses for all field sizes are normalized to $100 \%$ at the fixed depth $d_{m}$ of the $10 \mathrm{~cm} \times 10 \mathrm{~cm}$ reference field size. The actual maximum depth dose for some field sizes may therefore slightly exceed $100 \%$.

\section{equivalent field $v_{\theta q}$}

The equivalent field is defined as that square field which yields for a given physical quantity (e.g., phantom scatter correction factor at $d_{r e f}$ or PDD), the same value as the field under consideration. Note that for quantities, which are primarily related to phantom scatter (e.g., $S_{p}$ PDD, TPR), the sizes of the equivalent fields may mutually differ from those which are primarily related to collimator scatter.

\section{percentage depth dose PDD}

The percentage depth dose is the absorbed dose per monitor unit at a given depth $\mathrm{d}$ at the central beam axis, normalized to $100 \%$ at a depth which is equal to $d_{m}$ of the reference field size. $\operatorname{PDD}\left(v_{p}\right)=100 \% \times D\left(v_{p}, d\right) / D\left(v_{p}, d_{m}\right)$. 
0.

\section{phantom field size $v_{p}$}

For a photon beam impinging perpendicularly on a flat phantom surface, the field size $v_{p}$ is defined as the size of the field at the surface of the phantom with a source-surface distance equal to SAD. $v_{p}$ is one of the variables that determine the phantom scatter dose contribution to the point of measurement. Furthermore, the presence of shielding blocks in the beam or missing tissue influences the actual field size $v_{p}$ and therefore the phantom scatter contribution. Whenever the source-surface distance $f$ is not equal to SAD, the notation $v_{p, f}$ is used.

\section{phantom field size $v_{p, f}$}

The definition of the field size $v_{p, f}$ is the same as for $v_{p}$, but now the sourcesurface distance $f$ differs from SAD.

\section{phantom scatter correction factor $S_{p}$}

The phantom scatter correction factor $S_{p}$ is defined as the phantom scatter dose contribution for a specified collimator defined field size and a specified field size at the phantom surface, normalized to unity for the reference irradiation set-up. It is derived from the total scatter correction factor $S_{\mathrm{cp}}$ divided by the collimator scatter correction factor $S_{c}$ for the same collimator defined field size. The phantom scatter correction factor describes the influence of the scatter originating in the phantom only.

\section{quality index $Q$ I}

The quality index $\mathrm{Q}$ l is defined as the ratio of the electrometer reading per monitor unit with the ionization chamber at a depth of $20 \mathrm{~cm}$ in a water phantom, $R_{20}$, to the reading at a depth of $10 \mathrm{~cm}, R_{10}$, for a source-detector distance SDD equal to SAD and for a collimator setting $v_{c}$ of $10 \mathrm{~cm} \times 10 \mathrm{~cm}$. It is used as an indicator of the photon beam quality:

$\mathrm{Ql}=\mathrm{R}\left(\mathrm{v}_{\mathrm{c}, \mathrm{re}}, \mathrm{d}=20, \mathrm{SDD}=\mathrm{SAD}\right) / \mathrm{R}\left(\mathrm{v}_{\mathrm{c}, \text { ref }} \mathrm{d}=10, \mathrm{SDD}=\mathrm{SAD}\right)$.

\section{reference collimator defined field size $v_{c, r e f}$}

The reference field size $v_{c, \text { ref }}$ is that field size defined by the collimator setting which yields a field of $10 \mathrm{~cm} \times 10 \mathrm{~cm}$ at SAD.

\section{reference depth $d_{\text {ref }}$}

$d_{\text {ref }}$ is the reference depth, taken in this report equal to $10 \mathrm{~cm}$ irrespective of the radiation beam quality. In this way, uniformity of algorithms and continuity of beam data acquisition are obtained. For all clinically used megavoltage photon beams, $d_{r e f}$ is beyond the range of contaminating electrons.

\section{reference field size $v_{p, r e f}$}

The reference phantom field size $v_{p, r e f}$ is the phantom field size in the reference irradiation set-up. $v_{p, r e f}=10 \mathrm{~cm} \times 10 \mathrm{~cm}$. 


\section{reference irradiation set-up; ref (in subscripts)}

The reference irradiation set-up is defined as that irradiation geometry for which $\mathrm{d}=\mathrm{d}_{\mathrm{ref}}(=10 \mathrm{~cm}), \mathrm{v}_{\mathrm{p}}=\mathrm{v}_{\mathrm{p}, \mathrm{ref}}(=10 \mathrm{~cm} \times 10 \mathrm{~cm})$ and $\mathrm{v}_{\mathrm{c}}=\mathrm{v}_{\mathrm{c} \text {, ref }}(=10 \mathrm{~cm} \times 10$ $\mathrm{cm}$ ). As a consequence, $f=f_{\text {ref }}=S A D$.

\section{reference tray transmission factor $T F_{\text {ref }}$}

The reference tray transmission factor $\mathrm{TF}_{\mathrm{ref}}$ is the tray transmission factor, determined in the reference irradiation set-up. $T F_{\text {ref }}$ can be measured in a full scatter phantom or in a mini-phantom.

\section{reference wedge transmission factor $W F_{\text {ref }}$}

The reference wedge transmission factor $W F_{\text {ref }}$ is the wedge transmission factor, determined in the reference irradiation set-up. $W F_{\text {ref }}$ should be measured in a full scatter phantom.

\section{relative depth dose $R D D$}

The relative depth dose is the absorbed dose per monitor unit for a given depth at the central beam axis, normalized to unity at the reference depth $d_{\text {ref }}$,

$\operatorname{RDD}\left(v_{p}, d, f\right)=D\left(v_{p}, d, f\right) / D\left(v_{p}, d_{r e f}, f\right)$.

\section{relative tray transmission factor RTF}

The relative tray transmission factor is the tray transmission factor, determined at the reference depth for a given field size and tray-phantom distance, relative to the tray transmission factor for the reference irradiation set-up: RTF( $\left.v_{c}, T P D\right)$ $=T F\left(v_{c}, T P D\right) / T F_{r e f}$. The relative tray transmission factor describes the variation of the tray transmission factor with variations of field size and tray-phantom distance. RTF can be measured in a full scatter phantom or in a mini-phantom.

\section{relative wedge transmission factor $R W F$}

The relative wedge transmission factor is the wedge transmission factor, determined at the reference depth for a given field size and SSD, relative to the wedge transmission factor for the reference irradiation set-up: $R W F\left(v_{c}, f\right)=$ $W F\left(v_{c}, f\right) / W F_{r e f}$. The relative wedge transmission factor factor describes the variation of the wedge transmission factor with variations of field size and source-surface distance. RWF can be measured in a full scatter phantom as well as in a mini-phantom.

\section{solid angle $\Omega$}

The solid angle $\Omega$ is the angle which is geometrically defined by the point of measurement in the phantom and the surface of the flattening filter as observed from that point. The solid angle determines the amount of photons which is scattered in the flattening filter of the treatment machine in the direction of that point of measurement. 
0 .

\section{tissue-phantom ratio TPR}

The tissue-phantom ratio TPR at a point in a phantom irradiated by a photon beam is the total absorbed dose per monitor unit at that point, divided by the absorbed dose per monitor unit in the same point, but with the surface of the phantom moved in such a way that the point is at a specified reference depth.

\section{total scatter correction factor $S_{c p}$}

The total scatter correction factor is defined as the absorbed dose per monitor unit measured in a full scatter water phantom at the reference depth and the reference source-surface distance for a specified collimator defined field size $v_{c}$ and a specified field size at the phantom surface $v_{p}$, normalized to unity for the reference irradiation set-up.

$S_{c p}\left(v_{c}, v_{p}\right)=S_{c p}\left(v_{c}, v_{p}, d_{r e f}, f_{r e f}\right)=D\left(v_{c}, v_{p}, d_{r e f}, f_{r e f}\right) / D\left(v_{c, r e f}, v_{p, r e f}, d_{r e f}, f_{r e f}\right)$.

The total scatter correction factor describes the influence on the dose of both the collimator setting and the phantom field size.

\section{tray transmission factor TF}

The tray transmission factor is the ratio of the dose per monitor unit at the reference depth in a given field with a tray, to the dose per monitor unit in the same field without a tray in the beam. For a given tray, this ratio is slightly dependent on the field size and on the distance from the tray to the phantom surface, TPD: TF $\left(v_{c}, v_{p}, d_{r e f}, T P D\right)=D_{\text {tray }}\left(v_{c}, v_{p}, d_{r e f}, T P D\right) / D_{\text {open }}\left(v_{c}, v_{p}, d_{r e f}, T P D\right)$.

\section{wedge transmission factor WF}

The wedge transmission factor is the ratio of the dose per monitor unit at the reference depth in a specified field with a wedge present in the beam, to the dose per monitor unit in the same field without a wedge. WF $\left(v_{c}, v_{p}, d_{r e f}, f\right)=$ $D_{\text {wedge }}\left(v_{c}, v_{p}, d_{r e f} f\right) / D_{\text {open }}\left(v_{c}, v_{p}, d_{r e f}, f\right)$. The wedge transmission factor is dependent on field size and SSD. 
0. 


\section{Introduction}

The determination of the dose delivered to a patient receiving radiotherapy consists of several steps, each one introducing uncertainties in the reported dose value. To obtain an optimal dose with respect to tumour control and damage to normal tissues, the overall uncertainty in the dose has to be small. A value of about $3.5 \%$ (1 SD) has been proposed by several authors $[7,19,48]$. This can not always be achieved in clinical practice [16]. However, each source of error in the dose delivery has to be minimized. The calculation of the dose at a specified point, or the determination of the number of monitor units necessary to deliver a given dose, is one of the sources of uncertainty, especially when non-square, blocked, wedged or asymmetric fields are involved or a source-surface distance different from the reference one is applied.

One of the essentials in preparing a radiation treatment is the transformation of the prescribed dose into the appropriate radiation units of the treatment machine. In the case of a linear accelerator, these radiation units are measured by a monitor, which has to be calibrated in a predefined standard set-up, described as the reference condition. The dose at a particular point in a water phantom in a non-reference treatment set-up will be different. Scatter correction factors that mathematically describe these differences are used to translate the prescribed dose into a number of monitor units. This report describes the determination and use of these scatter correction factors for megavoltage photon beams.

In general, the output of a linear accelerator is adjusted in such a way that 100 monitor units (MU) correspond to a dose delivery of $1 \mathrm{~Gy}$ at a calibration point in a water-phantom under reference irradiation conditions: usually a symmetrical open field of $10 \mathrm{~cm} \times 10 \mathrm{~cm}$, defined at the phantom surface and at an SSD equal to the source-axis distance. The calibration point is usually chosen on the central beam axis at the depth of maximum absorbed dose $d_{m}$ in the $10 \mathrm{~cm} \times 10$ $\mathrm{cm}$ field.

Note: for ${ }^{60}$ Co therapy machines the output is expressed in units of dose per unit of time, e.g. Gy. $\mathrm{min}^{-1}$ at the date of calibration for specified calibration conditions.

The ratio of Gy to $\mathrm{MU}$ or time units depends on several parameters such as field size, depth, and SSD. The magnitude of the change with field size can be taken into account in dose calculations by applying the total scatter correction factor, which is defined as the absorbed dose per monitor unit, measured at the reference depth and reference SSD for a specified collimator setting and a specified field size at the phantom surface, normalized to unity for the reference collimator setting and a reference field size at the phantom surface. If the 
reference depth is taken equal to $d_{m}$, the total scatter correction factor is equal to what is commonly called the field size correction factor or output factor [8].

Recently, the influence of the geometry of the field size and the presence of blocks on the total scatter correction factor has been discussed by a number of authors $[14,25,27,33-34,36-39,43-46,54,64]$. It is generally agreed that the total scatter correction factor has to be separated into a collimator and a phantom scatter correction factor in order to improve the accuracy of dose calculations. These factors are related to the contributions of the collimator and phantom scatter to the output (in Gy/MU) of the treatment machine at a certain reference depth $[1,25,34,45,51,54,64]$. Several authors proposed a reference depth of 5 or $10 \mathrm{~cm}$, depending on whether the quality index $\mathrm{Ol}[17,47]$ of the photon beam is smaller or larger than $0.75[1,64]$, because then these depths are beyond the range of contaminating electrons. These electrons, originating in the head of the treatment machine, can influence the dose to superficial tissues in a rather unpredictable way. At larger depths, the influence of these electrons on the scatter correction factors is negligible. These reference depth values correspond to the phantom depth for the calibration, as proposed in a number of dosimetry protocols $[10,28,47]$. Furthermore, a high accuracy of the absorbed dose at the reference depth of 5 or $10 \mathrm{~cm}$ is clinically more relevant than at depth $d_{m}$. However, the use of two different reference depths for different beam qualities leads to a discontinuity in the description of the behaviour of the scatter factors as a function of beam quality. This discontinuity is eliminated by using a single reference depth of $10 \mathrm{~cm}$, irrespective of the photon beam quality.

Several methods to separately measure the phantom and collimator scatter components of the total scatter correction factor have been described $[42,64]$. This report adopts the use of a narrow cylindrical beam-coaxial phantom (miniphantom) for the measurement of the collimator scatter contribution. In combination with measurements in a full scatter phantom, the phantom scatter contribution can be derived.

The determination of these scatter correction factors is time consuming, due to the large number of possible field sizes in combination with beam modifiers. Limitation of the number of measurements can be obtained by expressing the scatter correction factors as a function of a limited set of parameters such as treatment machine and beam quality. A further reduction can be obtained if interpolation algorithms are applied.

The aim of this report is to describe the separation of the total scatter correction factor into its component parts. A coherent system of expressions is presented for the use of these scatter factors in the calculation of monitor units in clinical situations. The report considers the use of shielding blocks and wedges in a symmetrical set-up of the collimating jaws. Field sizes are defined and used for beams directed perpendicularly to the surface. Oblique incidence and the use of asymmetric collimating jaws and multi-leaf collimator set-ups are not considered. 
1.

Measured data are presented for photon beam qualities ranging from ${ }^{60} \mathrm{Co}$ to 25 $\mathrm{MV}$, for a large number of treatment machines. The reference depth is taken equal to $10 \mathrm{~cm}$, irrespective of the quality index of the photon beam.

The report recommends performing measurements and the handling of beam data in such a way that the uncertainty in the calculation of the number of monitor units for clinical situations is considerably smaller than $3.5 \%$ (1 SD), preferably smaller than 1\% (1 SD). Whenever possible, reference is made to interpolation algorithms with which a user can reduce the number of measurements necessary to obtain the required accuracy.

Section 2 of this report discusses the basic definitions and principles of this approach and the applicability of the methods to calculate the dose in megavoltage beams for square fields at arbitrary depths and SSDs. The influence of field elongation and the presence of a tray, blocks or wedges is discussed in section 3. Experimental methods and the results of a large number of measurements are given in sections 4 and 5 , respectively. The recommendations are summarized in section 6. Additional information is presented in the Appendices.

In this report the definitions of beam parameters and the reference geometry are based on a beam set-up involving a fixed $S S D$ technique, and not on an SAD or isocentric approach. In a water phantom, percentage depth dose data can be more easily measured than, for example, tissue-phantom ratio data, because the water level can be kept constant during the measurements. If TPR data are required for the calculations, the generally applied procedure is to perform PDD measurements and convert them into TPR data using conversion rules. Conversion rules have been published for this purpose $[8,9]$. Because the reference conditions are different for both approaches, care must be taken not to confuse the data. The definitions of the quantities and the relations between them in the fixed SSD (or PDD) approach and the isocentric (or TPR) approach are discussed in more detail in [71]. A brief summary is presented in Appendix 8.3. Whenever the quantities with definitions in the isocentric approach are used, the superscript iso is added.

The formalism presented in this report for the determination and use of scatter correction factors is similar to the procedure recommended in a recent ESTRO booklet "Monitor unit calculation for high energy photon beams" [18]. In that report, all the measurements and calculations needed for the determination of absorbed dose along the central axis per monitor unit are listed. Output factors are also defined at $10 \mathrm{~cm}$ depth and both the isocentric set-up and the fixed SSD approach are allowed. Consequently, the use of tissue-phantom ratios as well as relative depth doses are recommended and both formalisms are described. The ESTRO booklet gives an extensive description of the number of intermediate steps for the calculation of the number of monitor units for open beams, blocked beams, wedged beams, and beams at other distances than the 
1.

reference distance. Apart from differences in nomenclature, other differences with the ESTRO booklet are that in the present report no introduction is needed of a new quantity, the volume scatter ratio, and that in this report a wide variety of measured data is presented. 


\section{Collimator and phantom scatter correction factors for square fields}

\subsection{Definitions and principles}

\section{Principles of separation and measurement of scatter correction factors}

In the concept adopted in this report, the dose at a certain point in an irradiated phantom is attributed to two different origins.

First, there is the energy fluence of primary, unscattered photons which originate from the target, and of photons scattered somewhere in the head of the treatment machine. For practical reasons, no attempt is made to separate contributions from the various components of the treatment head, such as the flattening filter and collimator parts. It is assumed that the variation of these components with field size can be described with the desired accuracy by using only one factor, the collimator scatter correction factor.

Second, there is a contribution to the dose from radiation which is scattered within the phantom. The influence of the field size at the phantom surface on this contribution is described by the phantom scatter correction factor.

Scatter correction factors are defined as ratios of dose values, relative to values for a specified reference irradiation condition. The reference situation should reflect a clinically relevant geometry within the phantom, be easily accessible for measurement, and be free of disturbing influences. The use of only one reference situation is recommended, irrespective of photon beam quality or machine type. In this way, uniformity and continuity in presenting data as a function of beam quality is obtained.

\section{Total scatter correction factor $S_{c p}$}

The total scatter correction factor $\mathrm{S}_{\mathrm{cp}}\left(\mathrm{v}_{\mathrm{c}}, \mathrm{v}_{\mathrm{p}}, \mathrm{d}_{\mathrm{ret}}, \mathrm{f}_{\mathrm{ref}}\right)$ is defined as the ratio of the absorbed dose per monitor unit measured at the reference depth $d_{r e f}$, a reference source-surface distance $f_{r e f}$ for a specified collimator defined field size $v_{c}$ and $a$ specified field size at the phantom surface $v_{p}$, normalized to unity for the reference collimator setting and reference field size at the phantom surface. $v_{c}$ sets the field size at the SAD and $v_{p}$ the field size at the phantom surface. The reference values of the parameters $v_{c, r e f}=10 \mathrm{~cm} \times 10 \mathrm{~cm}$ and $v_{p, r e f}=10 \mathrm{~cm} \mathrm{x}$ $10 \mathrm{~cm}$, can only be realized if the source-surface distance is equal to SAD (for most megavoltage units $S A D$ is equal to $100 \mathrm{~cm}$ ). If, in addition, the depth in the phantom is set equal to the reference depth, $\mathrm{d}_{\text {ref, }}$ the dose measurement setup is referred to as the reference irradiation set-up. The dose, measured under reference conditions, is then $D_{\text {ref }}=D\left(v_{c, \text { ref }}, v_{p, r e f}, d_{r e f}, f_{r e f}\right)$ and the total scatter correction factor $S_{c p}$ can be written as: 


$$
S_{c p}\left(v_{c}, v_{p}, d_{r e f}, f_{r e f}\right)=D\left(v_{c}, v_{p}, d_{r e f}, f_{r e f}\right) / D_{r e f}
$$

With respect to the choice of the reference depth it should be noted that at shallow depths the dose is influenced by electrons contaminating the photon beam, of which the contribution varies markedly with the setting of and the distance from the collimator. These electrons originate from the target, the flattening filter, and from the beam defining parts of the treatment head. When hitting the phantom, the electrons are totally absorbed in the first few centimeters. Therefore, contaminating electrons do not contribute to the dose at depths where most tumours are found in clinical practice. Furthermore, prediction of the contribution of contaminating electrons to the dose for a particular irradiation geometry is a complex matter. For these reasons, the reference depth should be chosen in such a way that the effect of the contaminating electrons is negligible.

Note, that the total scatter correction factor is strictly defined for a depth equal to the reference phantom depth and for an SSD equal to the SAD. This definition is more restrictive than the definitions given in earlier publications of this task group [64] and of others [25,34] (see Appendix 8.1). Furthermore, due to the restrictions of the reference conditions of the scatter correction factor, the parameters $d_{\text {ref }}$ and $f_{\text {ref }}$ in equation (2.1.1) are fixed and can therefore be removed from the notation of $S_{c p}$ without misunderstanding.

The total scatter correction factor $S_{c p}\left(v_{c}, v_{p}\right)$ is now written as the product of two components: the collimator scatter correction factor $S_{c}\left(v_{c}, v_{p}\right)$ and the phantom scatter correction factor $S_{p}\left(v_{c}, v_{p}\right)[1,25,34,45,51,54,64]$. In a general form:

$$
S_{c p}\left(v_{c}, v_{p}\right)=S_{c}\left(v_{c}, v_{p}\right) \times S_{p}\left(v_{c}, v_{p}\right)
$$

\section{Collimator scatter correction factor $S_{c}$}

$S_{c}\left(v_{c}, v_{p}\right)$ reflects the change in the energy fluence in air per monitor unit of the scattered photons emitted from the head of the accelerator due to a varying opening of the collimator jaws, relative to the reference irradiation set-up $[34,64]$. Variation of the collimator setting results, on the one hand, in variable amounts of photons scattered to the point of measurement from the primary collimator, the flattening filter and, on the other hand, in variable amounts of radiation scattered backwards from the collimator jaws into the monitor chamber, situated in the head of the treatment machine $[26,33,37-39,45]$. The variable amount of scattered radiation reaching the point of measurement is for a large part related to the solid angle $\Omega$, which is the angle geometrically defined by the point of measurement in the phantom and the surface of the flattening filter that can be seen from that point. It is the setting of the collimator jaws, given by $v_{c}$, which determines that surface. Thus, $v_{c}$ is directly related to the solid angle $\Omega$ and partly determines the change in the energy fluence in air per 
monitor unit. In case of extreme blocking of the field with blocks on a tray, certain parts of the flattening filter may be hidden and the solid angle $\Omega$ has to be used as a variable in the calculation procedures instead of $v_{c}$ (see section 3 and Appendix 8.5).

Like the total scatter correction factor $S_{c p}$ the collimator scatter correction factor $S_{c}\left(v_{c}, v_{p}\right)$ is defined at the reference depth. Concerning the choice of the reference depth, note that the variation of the energy fluence in air per monitor unit is primarily determined by the volume of the primary collimator and the flattening filter within the solid angle $\Omega$. The influence of the depth in the phantom on this volume is almost negligible [64]. $S_{c}$ can, therefore, be considered to be independent of the choice of $d_{\text {ref }}$ as long as $d_{r e f}$ is larger than the range of the contaminating electrons. For the same reason, the collimator scatter correction factor is hardly influenced by the choice of the SSD within the clinical useful range [64].

The primary energy fluence in air cannot be measured directly. However, it can be deduced relatively to the energy fluence under reference conditions by using a mini-phantom, as proposed by this task group (van Gasteren et al. [64]). In such a mini-phantom, the ionization chamber is placed in an upright position, at a depth of $10 \mathrm{~cm}$. The collimator scatter correction factor $S_{c}$ is then the ionization reading for the collimator defined field size $v_{c}$, normalized to unity for the reference field $v_{c, r e f}$.

$S_{c}$ is a machine related factor, which can be assumed to depend only on the collimator setting which is defined by the field size at $S A D$. Because $S_{c}$ is independent of the choice of SSD and depth in the mini-phantom, as long as this depth is larger than the range of contaminating electrons, $S_{c}$ can be written as a function of $v_{c}$ only: $S_{c}\left(v_{c}\right)$.

\section{Phantom scatter correction factor $S_{p}$}

The phantom scatter correction factor $S_{p}$ accounts for the contribution of the radiation scattered in the phantom material to the dose at the point at depth $d_{\text {ref }}$ on the central axis, relative to the phantom scatter contribution in the reference irradiation set-up. The amount of scattered radiation reaching the point depends on the volume of the irradiated phantom material. Therefore, $S_{p}$ is a phantom related factor with a dependence on the irradiated volume, which is accounted for by writing $S_{p}$ as a function of the field size at the surface of the phantom. The increase of phantom scatter with depth makes $S_{p}$ dependent on the choice of the reference depth. It is, however, important to note that $S_{p}$ is defined only for one reference depth, $d_{\text {ref }}$, equal to the one chosen for $S_{c p}$ and $S_{c}$. For this reason $d_{r e f}$ does not need to be given as a parameter of $S_{p}$.

Another important factor, influencing the amount of scattered radiation, is the quality of the photon beam, indicated by the quality index, QI. $S_{p}$ varies with the nominal photon beam energy [64]. For a given beam, some changes in the 
photon energy spectrum will occur, when for example beam modifiers such as wedges and heavy metal compensators are used in the field. This may influence to some extent the magnitude of $S_{p}$, but these effects are small, even for wedged photon beams [22]. In addition, it can be assumed that the influence of commonly applied blocking trays on the value of $\mathrm{S}_{p}$ can be neglected. Therefore, for a beam with a given $Q$ I, $S_{p}$ will be written as a function of $v_{p}$ only, that is: $\mathrm{S}_{\mathrm{p}}\left(\mathrm{v}_{\mathrm{c}}, \mathrm{v}_{\mathrm{p}}\right)=\mathrm{S}_{\mathrm{p}}\left(\mathrm{v}_{\mathrm{p}}\right)$.

\section{Reference depth, $d_{\text {ref }}$}

The reference depth $d_{r e f}$ is chosen to be $10 \mathrm{~cm}$ for all photon beam qualities addressed in this report, i.e. from ${ }^{60} \mathrm{Co}$ to $25 \mathrm{MV}$. As indicated previously, the use of a single reference depth ensures continuity of $S_{p}$ as a function of beam quality. The depth of $10 \mathrm{~cm}$ is chosen to obtain the highest accuracy in the calculation of treatment times and monitor units at depths which are clinically most relevant. For the same reason, this reference depth is chosen in most dosimetry protocols to measure the output of treatment machines. Note that the influence of contaminating electrons in the beam on the dose at shallow depths is not ignored, but taken into account by the percentage depth dose or relative depth dose (PDD or RDD; see section 2.2 for the definition of RDD) values at these depths.

\section{Summary}

As a result of these considerations, the total scatter correction factor $S_{c p}\left(v_{c}, v_{p}\right)$ is given by:

$$
S_{c p}\left(v_{c}, v_{p}\right)=S_{c}\left(v_{c}\right) \times S_{p}\left(v_{p}\right)
$$

$S_{c p}$ and $S_{c}$ data can be obtained by using the measurement method shown in figure 2.1. $S_{p}$ can be obtained from equation (2.1.3). The measurement conditions are discussed in more detail in section 4 of this report.

In the following sections, the use of the scatter correction terms will be discussed for dose calculations at arbitrary depths and arbitrary SSDs. In most clinical cases, the situation will be more complex, because in addition to an arbitrary depth and SSD, customized blocks and other beam modifying devices are also used. However, it is important to keep in mind that the same methodology can still be applied: a separation of the influence of collimator and phantom related scatter to the dose at the reference point. Factors influencing the energy fluence from the head of the treatment machine to the phantom surface are taken into account by the collimator scatter correction factor $S_{c}$, which has to be measured using the mini-phantom as described in this report: For specific cases, individual measurements of $S_{c}$ may be needed. Factors influencing the phantom volume irradiated by the beam are taken into account by the phantom scatter correction factor $S_{p}$. Because the variation of $S_{p}$ with variations of the mean energy of megavoltage photon beams is slow [56], in 
2.

general no further measurements will be needed for the determination of $S_{p}$ if beam modifying devices are applied.

Some guidelines for the use of the scatter correction factors in more complex cases will be given in section 3 .

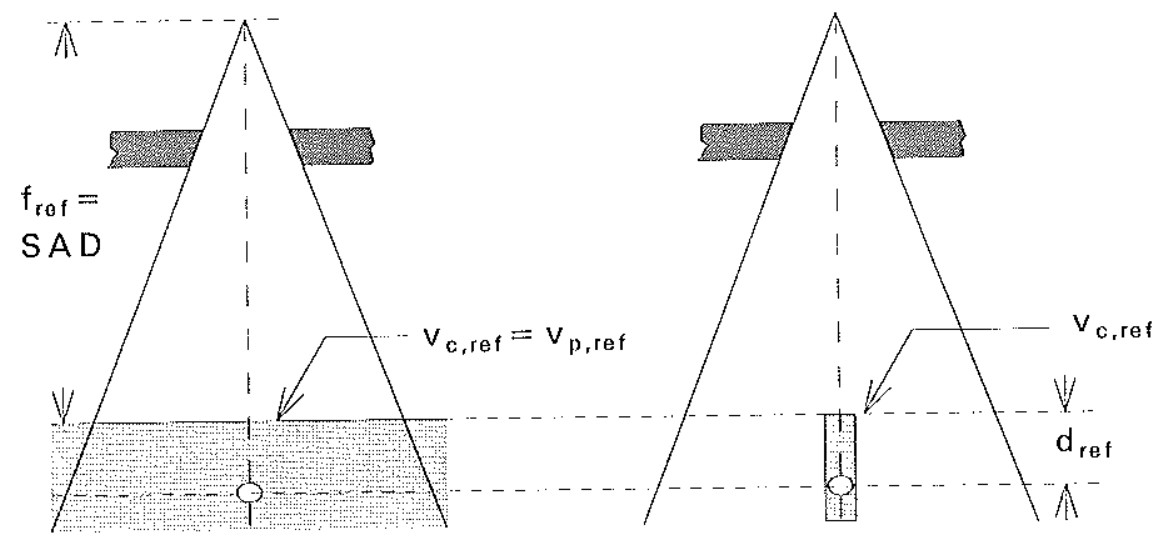

a)

b)

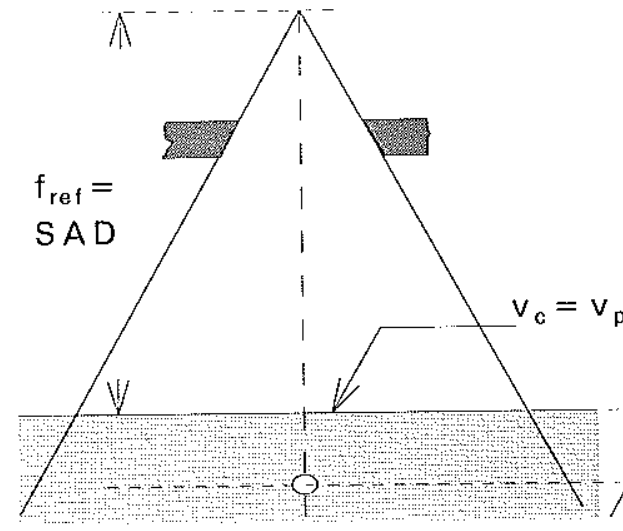

c)

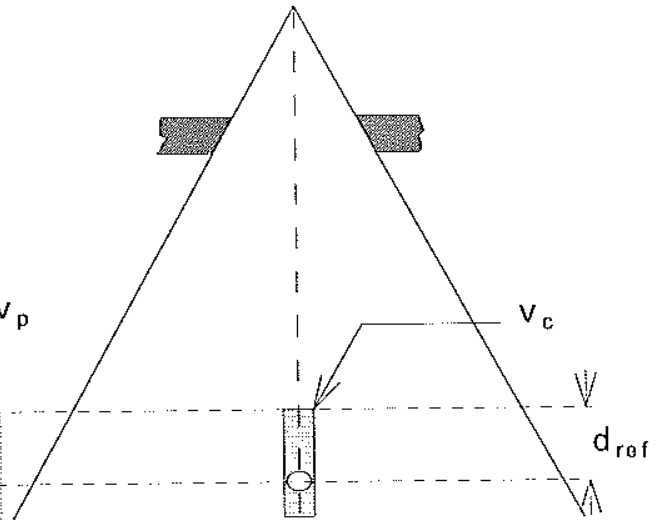

d)

Figure 2.1 Measurement of the scatter correction factors $S_{c p}(a, c)$ and $S_{c}(b, d) . S_{c p}$ and $S_{c}$ data are derived directly from measurements relative to the reference irradiation set-up, using a full scatter water phantom and a mini-phantom, respectively. Phantom scatter data $S_{p}$ are then obtained by dividing $S_{c p}$ data by $S_{c}$ data. $S S D$ is taken equal to $S A D\left(=f_{\text {ref }}\right.$ usually $\left.100 \mathrm{~cm}\right)$; the depth of measurement $d$ is taken equal to $d_{r e f}(=10 \mathrm{~cm})$. 


\subsection{Calibration and reference irradiation conditions}

For calibration purposes, the treatment machine is set in such a way that $v_{c}=$ $v_{c, r \text { er }}$ and $v_{p}=v_{p, r e f}$ at source-surface distance $f$ equal to SAD $\left(=f_{r e f}\right)$. Usually, the monitor chamber of the treatment unit is calibrated to deliver a dose $D_{\text {cal }}$ per monitor unit (MU) of $1 \mathrm{cGy}$ at the depth $\mathrm{d}_{\mathrm{m}}$ of maximum absorbed dose on the central axis of the beam. This calibration setting is used throughout this report, although other calibration settings are possible.

We can then write (see figure 2.2):

$$
D_{\text {cal }}=D\left(v_{c, \text { ret }}, v_{p, \text { ret }}, d_{m}, f_{\text {ref }}\right)=1 \mathrm{cGy} \text { per monitor unit }
$$

Note: for ${ }^{60}$ Co therapy machines the output is expressed in units of dose per

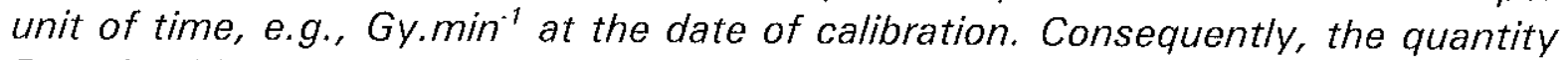
$D_{c a l}$ should be expressed in corresponding units. For these types of therapy machines, this is implicitly assumed in the following sections.

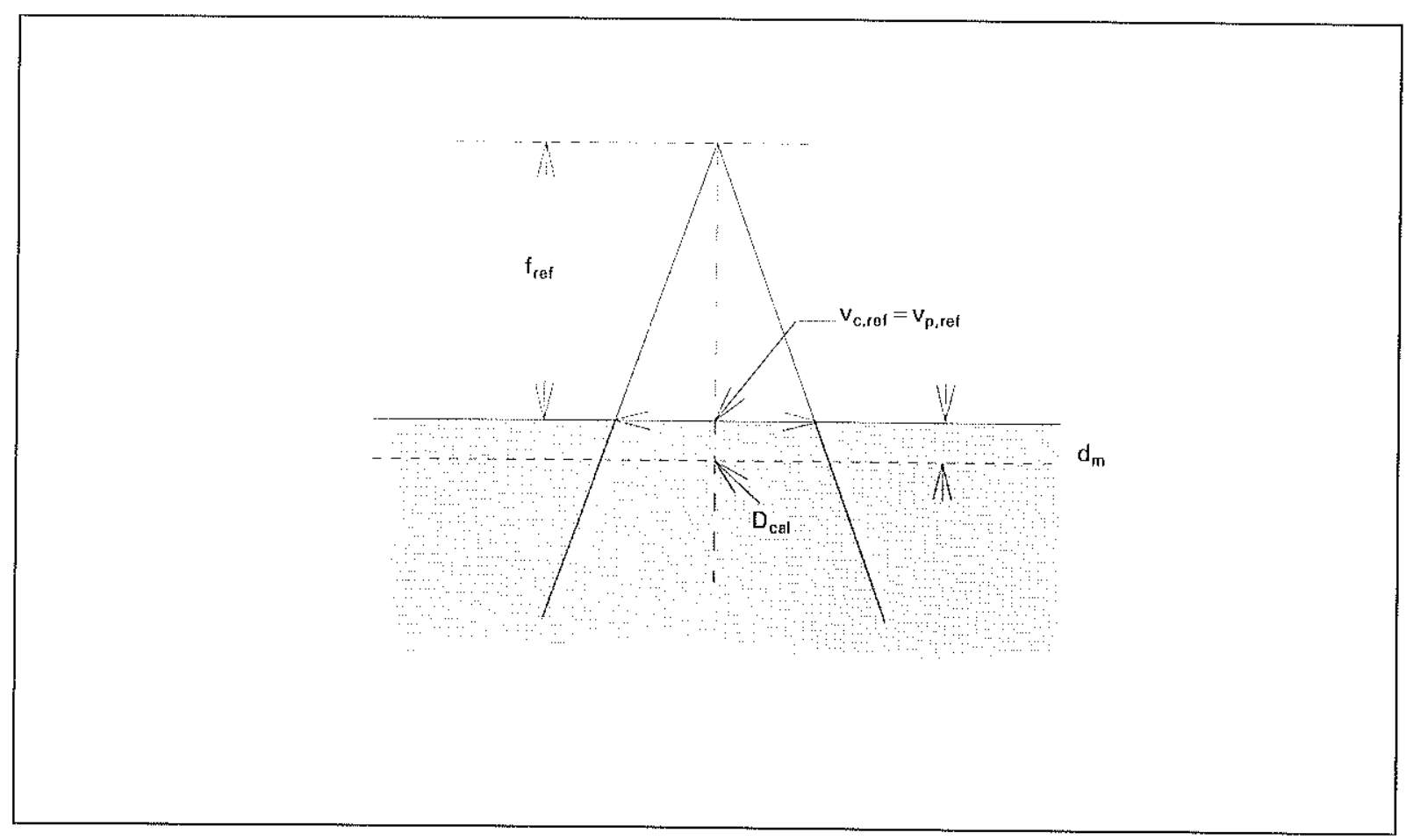

Figure 2.2 The monitor chamber of the treatment machine is calibrated to deliver a dose of 1 cGy per monitor unit at a depth of maximum absorbed dose, $d_{m}$ in the reference field: $D_{c a l}=D\left(v_{c_{c} \text { ref }}, v_{p, r o f}, d_{m,} f_{\text {ref }}\right)$, with $v_{c, \text { ef }}=v_{p, r \text { ref }}=10 \mathrm{~cm} \times 10 \mathrm{~cm}$ and $f_{\text {ref }}=100 \mathrm{~cm}$.

Dosimetry protocols recommend determining the absorbed dose in a beam at a depth $d_{\text {ref }}[28,47]$. In order to relate the calibration dose at $d_{m}$ to the dose at $d_{r e f}$, 
2.

use is made of the percentage depth dose of the calibration field, $\operatorname{PDD}\left(v_{\mathrm{p}, \mathrm{ret}}, \mathrm{d}_{\mathrm{ref}}, \mathrm{f}_{\mathrm{ref}}\right)$, see figure 2.3,

$$
D_{\text {ref }}=D_{\text {cal }} \times \operatorname{PDD}\left(v_{p, r o t}, d_{\text {ref }}, f_{\text {ref }}\right) / 100
$$

which can be rewritten as:

$$
D_{\text {ref }}=D_{\text {cal }} / R D D\left(v_{p, r e f}, d_{m}, f_{r e f}\right)
$$

This expression introduces the relative depth dose, RDD. For a given sourcesurface distance $f$ and a given field $v_{p}$, RDD is defined as the absorbed dose at a certain depth $d$, normalized to unity at the reference depth $d_{r e f}$. Its behaviour is analogous to that of the PDD of the beam at the same SSD, although its normalization is at $d_{\text {ref }}$ instead of $d_{m}$. The RDD can be derived from PDD data using:

$$
\operatorname{RDD}\left(v_{p}, d, f\right)=\operatorname{PDD}\left(v_{p}, d, f\right) / \operatorname{PDD}\left(v_{p}, d_{r e f}, f\right)
$$

From the definition it is clear that at depth $d=d_{\text {ref }}$ RDD is always equal to unity, irrespective of field size, $v_{p}$, or source-surface distance, $f$.

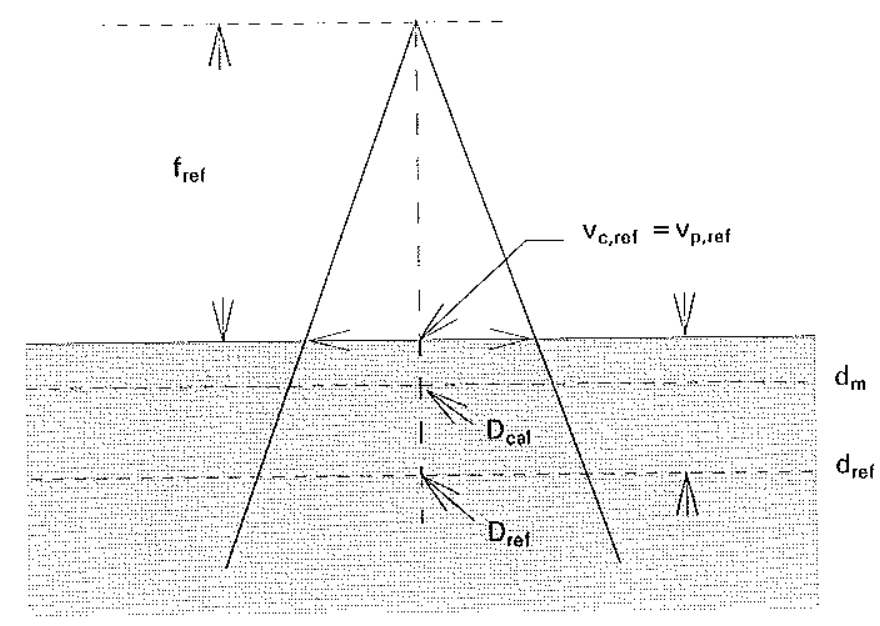

Figure 2.3 Dosimetry protocols recommend to calibrate a beam at the reference point in the reference field, i.e. at depth $d_{r e f}$ and field size $v_{c, r e f}=v_{p, r e f}=10 \mathrm{~cm} \times 10 \mathrm{~cm} ; f_{r e f}=100 \mathrm{~cm}$. 
2.

\subsection{Calculation of the dose under non-reference conditions}

\section{Beams with $S S D=f_{\text {ref }}$}

The absorbed dose at a point at the reference depth $d_{\text {ref }}$ in a beam with arbitrary field size $\left(v_{c}, v_{p}\right)$ and a reference source-surface distance $f_{\text {ref, }}$ is given by (see figure 2.4):

$$
D\left(v_{c}, v_{p}, d_{r e f}, f_{r e f}\right)=D_{\text {ref }} \times S_{c}\left(v_{c}\right) \times S_{p}\left(v_{p}\right)
$$

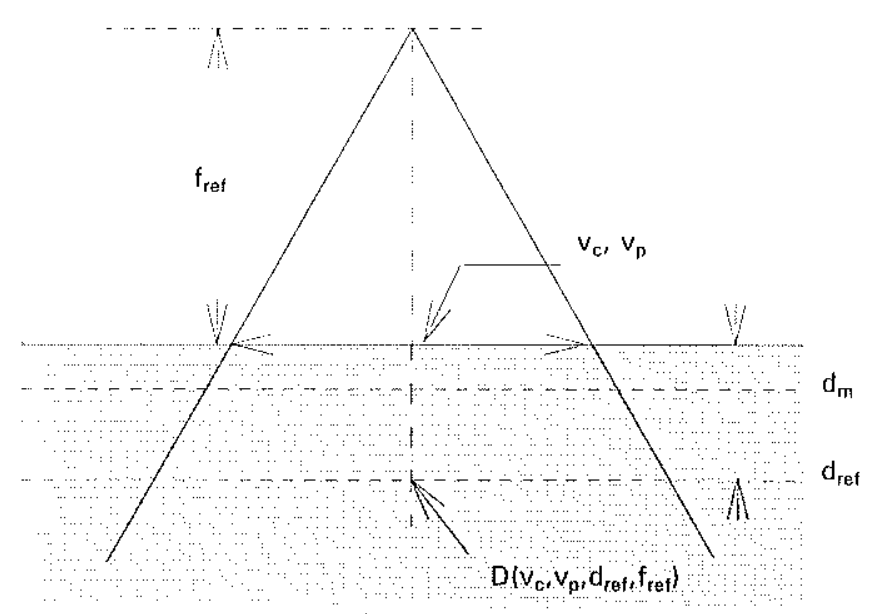

Figure 2.4 Dose in an arbitrary field at depth $d_{r e f}$ and SSD equal to SAD: $D\left(v_{c}, v_{p}, d_{r e f}, f_{r e f}\right)$. The quantities $S_{c}\left(v_{c}\right)$ and $S_{p}\left(v_{p}\right)$ are defined at depth $d_{r e t}$ and at source-surface distance $f_{r e f}$. 
2.

From the definition of the relative depth dose, the absorbed dose at other depths d can be written as (see figure 2.5):

$$
\begin{aligned}
D\left(v_{c}, v_{p}, d, f_{r e f}\right) & =D\left(v_{c}, v_{p}, d_{r e f}, f_{r e f}\right) \times \operatorname{RDD}\left(v_{p}, d, f_{r e f}\right) \\
& =D_{r e f} \times S_{c}\left(v_{c}\right) \times S_{p}\left(v_{p}\right) \times \operatorname{RDD}\left(v_{p}, d, f_{r e f}\right)
\end{aligned}
$$

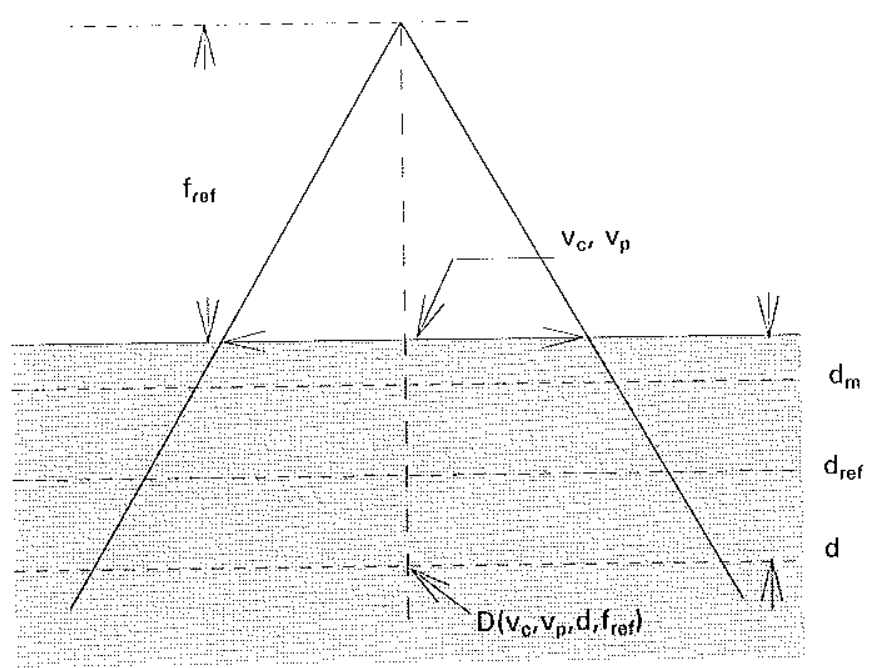

Figure 2.5 Dose in an arbitrary field at depth $d$ and with SSD equal to SAD: $D\left(v_{c}, v_{p}, d, f_{r e f}\right)$. 


\section{Beams with $S S D \neq f_{\text {rof }}$}

In the general case, when the SSD of a beam deviates from the reference SSD, the numerical value of the field size $v_{p, f}$ is no longer numerically equal to that of the collimator defined field size $v_{\mathrm{c}}$. This general situation is shown in figure 2.6. (Note that the term $v_{p, f}$ is used here for the field size defined at the phantom surface positioned at an arbitrary $f$; the term $v_{p}$ is reserved for the case when $f$ is equal to SAD. The term $v(d)$ is used to indicate the field size at depth d, i.e. at a source-to-point distance $f+d$.)

According to the definitions in this report, $v_{p, t}$ and $v_{c}$ can both be considered as those quantities describing the field size of the beam; see figure 2.6.a. Values of the collimator scatter correction factors can be derived directly from measurements of $S_{c}$, which will in general be tabulated as a function of $v_{c}$. However, values of the phantom scatter correction factor are determined at $f_{\text {ref }}$, and will in general be available in a format for this $f_{\text {ref }}$.

The phantom scatter contribution to the dose at depth d depends on the size of the field at that depth, $v(d)$, rather than on the field size at the surface. This observation was first discussed by Johns et al. [32] and was taken into consideration in the relations between depth dose characteristics at different SSDs (see Burns, Appendix B in reference [8]). Phantom scatter correction factors can thus be used from measurements made at a reference sourcesurface distance (see figure 2.6.b) for a field size at $f_{\text {ref }}$ that projects to the same field size $v(d)$ at depth $d$ as the field $v_{p, t}$.

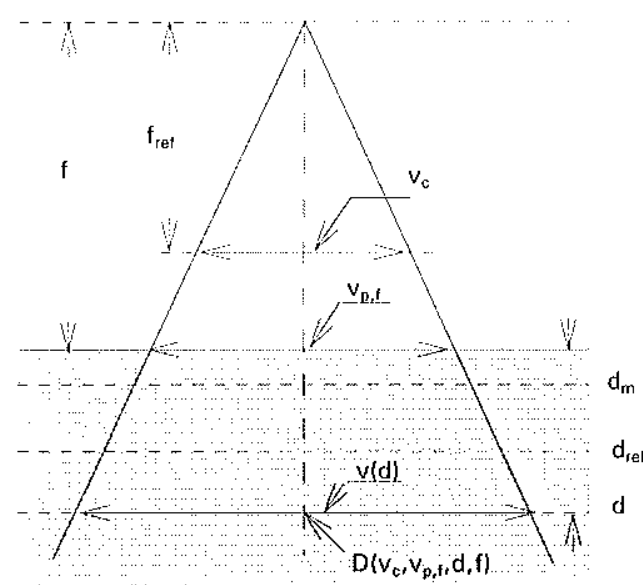

a)

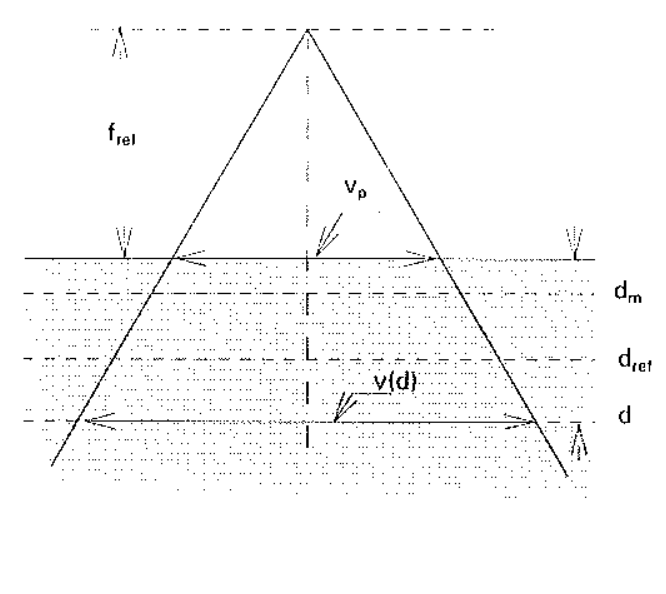

b)

Figure 2.6 Dose in an arbitrary field at depth $d$ and source-surface distance $f, D\left(v_{c}, v_{0, f}, d, f\right)$. Here, the relations between field size at depth $d, v(d), v_{c}, v_{p, f}$ and $v_{p}$ have to be taken into account (see text): $v_{p}=v_{o, f} / F$. 
In order to obtain the correct phantom scatter correction factor at depth $\mathrm{d}_{\text {ref }}$ a correction factor $F$ is needed to relate the field size at depth to the field size at the surface, taking into account the different beam divergence for the different SSDs. The dose at a point can be calculated with the following expression, of which a more detailed derivation can be found elsewhere [71]. Here, only the resulting relation is shown between the dose at an arbitrary depth and an arbitrary SSD and the dose at reference conditions, $D_{\text {ref }}$ :

$$
\begin{aligned}
D\left(v_{c}, v_{p, f}, d, f\right)=D_{\text {ref }} & \times S_{c}\left(v_{c}\right) \times S_{p}\left(v_{p, f} / F\right) \times\left\{\left(f_{r e f}+d\right) /(f+d)\right\}^{2} \\
& \times R D D\left(v_{p, f} / F, d, f_{r e f}\right)
\end{aligned}
$$

or rather, with equation $(2.2 .3)$ relative to the calibration dose, $D_{\text {cal }}$ :

$$
\begin{aligned}
D\left(v_{c}, v_{p, f}, d, f\right)=D_{c a t} & \times S_{c}\left(v_{c}\right) \times S_{p}\left(v_{p, f} / F\right) \times\left\{\left(f_{r e f}+d\right) /(f+d)\right\}^{2} \\
& \times \operatorname{RDD}\left(v_{p, f} / F, d, f_{r e f}\right) / \operatorname{RDD}\left(v_{p, r \text { ref }}, d_{m}, f_{r e f}\right)
\end{aligned}
$$

In these equations, the factor $F$ corrects the field size to be used in the phantom scatter correction factor and in the RDD, according to $[8,9]$ :

$$
F=\frac{f_{\text {ref }}+d}{f_{\text {ref }}} \times \frac{f}{f+d}
$$




\subsection{Calculation of treatment time and monitor units in the fixed SSD formalism}

\section{Beams with $S S D=f_{\text {ref }}$}

For the situation where the source-surface distance equals SAD, the number of monitor units $N$, required to deliver a prescribed dose, $D_{\text {presc }}$, to the dose prescription point at any depth in an arbitrary field (i.e. with $v_{c}, v_{p}$, depth $d$, and $\left.f_{\text {ref }}\right)$, can now be calculated.

Substitution of $D_{\text {ref }}$ from equation (2.2.3) into equation (2.3.2) yields a dose of $\left.D_{\text {cal }} \times S_{c}\left(v_{c}\right) \times S_{p}\left(v_{p}\right) \times R D D\left(v_{p}, d, f_{r e f}\right) / R D D\left(v_{p, r e f}, d_{m}, f_{r e f}\right)\right\}$ per monitor unit.

In order to deliver the prescribed dose $D_{\text {presc }}$ at the specification point, $N$ monitor units are required according to:

$$
N=D_{\text {presc }} /\left\{D_{\text {cal }} \times S_{c}\left(v_{c}\right) \times S_{p}\left(v_{p}\right) \times R D D\left(v_{p}, d, f_{\text {ref }}\right) / R D D\left(v_{p, r e t}, d_{m}, f_{r e f}\right)\right\}
$$

\section{Beams with $S S D \neq f_{\text {ref }}$}

For the more general case, where an arbitrary source-surface distance $f$ is used, equation (2.4.1) can be rewritten using equation (2.3.5) to yield:

$$
\begin{aligned}
N=D_{\text {presc }} / & \left\{D_{\text {cai }} \times S_{c}\left(v_{c}\right) \times S_{p}\left(v_{p, f} / F\right) \times\left\{\left(f_{r e f}+d\right) /(f+d)\right\}^{2}\right. \\
& \left.\times R D D\left(v_{p, f} / F, d, f_{r e f}\right) / \operatorname{RDD}\left(v_{p, \text { ref }}, d_{m}, f_{r e f}\right)\right\}
\end{aligned}
$$


2.

\subsection{Calculation of monitor units in the isocentric formalism}

The isocentric set-up is widely used in external beam radiotherapy. In many institutions dose measurements are performed from which tissue-phantom ratios are derived as the basis for an isocentric calculation system. The reference irradiation conditions for these measurements are essentially different from those used in the fixed SSD formalism. In this paragraph, the basic definitions of the isocentric (or TPR) approach are given, together with the expression to calculate the number of monitor units for a certain prescribed dose; see also Appendix 8.3 [71].

Analogous to the fixed SSD situation, we consider the isocentric situations of figure 2.7 .

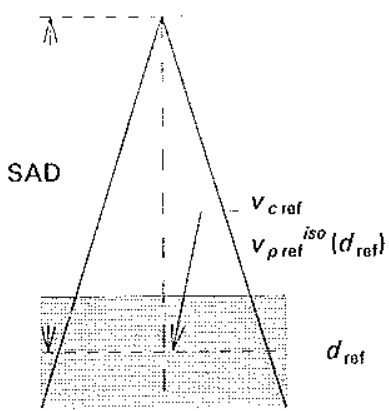

a)

$D_{i t}=D_{\text {ets }}^{\text {iso }}$

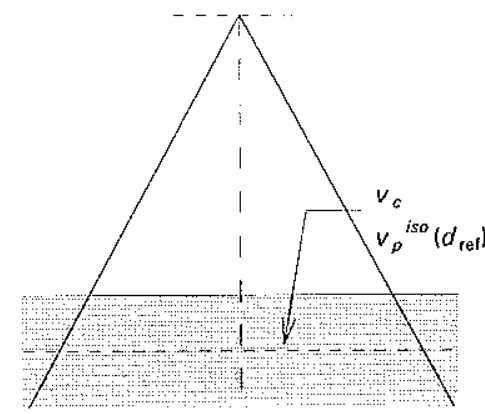

b)

$D_{b}=D_{\text {ret }}^{i s o} S_{c}^{i s o}\left\langle v_{c}\right\rangle S_{p}^{i s o}\left(V_{p}^{i s o}\left(d_{\text {tee }}\right)\right)$

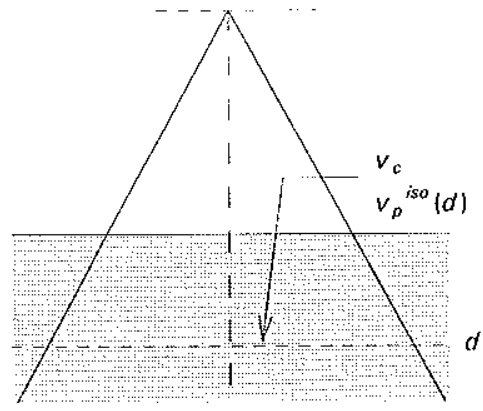

c)

$D_{c}=D_{\text {rel }}^{i s o} S_{c}^{i s o}\left(v_{c}\right) S_{p}^{i s o}\left(v_{p}^{i s o}\langle d\}\right)$ $\operatorname{TPR}\left(v_{p}{ }^{i s o}(d), d\right)$

Figure 2.7 The use of the tissue-phantom ratio in dose calculations.

The reference dose per monitor unit in the isocentric approach, $D_{\text {ref }}^{\text {iso }}$, is usually specified at the isocentre, at a reference depth, $\mathrm{d}_{\text {ref }}$, equal to $10 \mathrm{~cm}$, in an open beam with a field size of $10 \mathrm{~cm} \times 10 \mathrm{~cm}$ defined at the isocentre, which is in most cases at a distance from the focus (SAD) equal of $100 \mathrm{~cm}$.

The collimator scatter correction factor for the isocentric approach, $\mathrm{S}_{\mathrm{c}}$ iso, is measured in the same manner as in the fixed SSD approach, i.e. using the miniphantom with the detector at $10 \mathrm{~cm}$ depth, but now the detector is positioned at the SAD. As discussed in section 8.3, $S_{c}$ iso is equal to $S_{c}$. The phantom scatter correction factor for the isocentric approach, $S_{p}{ }^{\text {iso }}\left(v\left(d_{r e f}\right)\right)$, is derived from the ratio $\mathrm{S}_{\mathrm{cp}}$ iso $\left(\mathrm{v}_{\mathrm{c}}, \mathrm{v}\left(\mathrm{d}_{\mathrm{ref}}\right)\right) / \mathrm{S}_{\mathrm{c}}^{\text {iso }}\left(\mathrm{v}_{\mathrm{c}}\right)$, where $\mathrm{S}_{\mathrm{cp}}{ }^{\text {iso }}\left(\mathrm{v}_{\mathrm{c}}, \mathrm{v}\left(\mathrm{d}_{\mathrm{ref}}\right)\right)$ is measured in a full 
scatter phantom, again with the detector at the depth $d_{\text {ref }} . v\left(d_{\text {ref }}\right)$ is the field size at depth $d_{\text {ret }}$, i.e. at the isocentre.

\section{Beams with $S S D=S A D-d$}

For a point at the isocentre at any depth $d$, the absorbed dose per monitor unit for a collimator defined field size $v_{c}$, field size $v(d)$ at depth $d$ and a sourcesurface distance equal to SAD-d, is given by:

$$
D\left(v_{c}, v(d), d, S A D-d\right)=D_{\text {ref }}^{\text {iso }} \times S_{c}^{\text {iso }}\left(v_{c}\right) \times S_{p}^{\text {iso }}(v(d)) \times \operatorname{TPR}(v(d), d)
$$

The field size at the isocentre $v(d)$ also takes into account possible blocking of the beam.

The number of monitor units $N$ required to deliver the prescribed dose $D_{\text {presc }}$ at the specification point is now given by:

$$
N=D_{\text {presc }} /\left\{D_{\text {ref }}^{\text {iso }} \times S_{c}^{\text {iso }}\left(V_{c}\right) \times S_{p}^{\text {iso }}(v(d)) \times \operatorname{TPR}(V(d), d)\right\}
$$

\section{Beams with $S S D \neq S A D-d$}

For the more general situation where the dose specification point is not at the isocentre (i.e. Where the source-surface distance $f \neq S A D-d$ ) the inverse square law with respect to the SAD has to be introduced and the dose is written as:

$$
D\left(v_{c}, v(d), d, f\right)=D_{\text {ref }}^{\text {iso }} \times S_{c}^{\text {iso }}\left(v_{c}\right) \times S_{p}^{\text {iso }}(v(d)) \times \quad\{S A D /(f+d)\}^{2} \times T P R(v(d), d)
$$

and equation (2.5.2) becomes:

$$
N=D_{\text {presc }} /\left\{D_{\text {ref }}^{\text {iso }} \times S_{c}^{\text {iso }}\left(v_{c}\right) \times S_{p}^{\text {iso }}(V(d)) \times\{S A D /(f+d)\}^{2} \times \operatorname{TPR}(V(d), d)\right\}
$$

Note that the quantities used in these equations are derived from the isocentric, i.e. the TPR approach, and these should not be confused with those from the fixed SSD approach. The relations between $\mathrm{D}_{\text {ref }}$ iso and $\mathrm{D}_{\text {ref }} ; \mathrm{S}_{\mathrm{c}}$ iso and $\mathrm{S}_{\mathrm{c}} ; \mathrm{S}_{\mathrm{p}}$ iso and $S_{p}$ are given in Appendix 8.3 [71]. 


\section{Scatter correction factors for non-square fields, fields with wedges, compensators and trays}

\subsection{Rectangular and irregularly shaped fields}

\section{The collimator scatter correction factor $S_{c}$ in rectangular fields}

It has been shown by several authors that for rectangular fields the collimator scatter correction factor $S_{c}$, and therefore the total scatter correction factor for a given collimator setting, will be different if the upper and lower collimator jaws are interchanged $[24,57-59,62]$. This effect is commonly described as the collimator exchange effect, CEE. The effect originates from different amounts of radiation scattered backwards from the upper and lower collimator jaws into the beam monitor chamber $[14,18,24,41]$ and from differences in energy fluence of photons originating from the flattening filter reaching the point of interest [3]. The magnitude of the collimator exchange effect depends on the construction of the head of the treatment machine including such factors as the dimensions and material of the flattening filter, the presence of collimator satellites close to the monitor chamber, the distance between the upper collimator parts and the monitor chamber, and the presence of shielding material in the accelerator head.

Accurate calculation of the output of rectangular fields requires the determination of $S_{c}$ values for the large range of irradiation geometries used routinely in the clinic. A two-dimensional table can be constructed, often obtained from measured and partly interpolated or fitted data. The use of a 2-D table is in principle simple and accurate. It can easily be incorporated in calculation systems using present-day computer technology. The beam data in this report have been presented in this way. However, the determination of the 2-D table of $S_{c}$ values is time-consuming and, therefore, methods to minimize the number of measurements might be considered $[24,31,57-59,62]$.

\section{Analytical approximations}

Most methods given in the literature for estimating $S_{c}$ of a particular rectangular field are based on the determination of the equivalent square field yielding the same $S_{c}$ value $[4,55,57,62]$. In general, equations are proposed for which parameters have to be determined from $S_{c}$ measurements for a limited number of square and rectangular fields. Various methods, based on the equivalent square field method, have been analyzed by Jager et al. [31] for a large number of treatment machines and photon beam qualities with respect to the accuracy in estimating the $S_{c}$ value of rectangular fields. The data-fitting method described in their paper is at the moment the most accurate one. Applying that methodology, $S_{c}$ values can be estimated with deviations smaller than $0.8 \%$ between the estimated and actual $S_{c}$ values for all collimator settings and for various types of treatment machines. However, the authors concluded that a model based on the physical characteristics of a treatment head might be helpful 
3.

for the accurate calculation of $\mathrm{S}_{\mathrm{c}}$ values for all treatment machines in clinical use (Appendix 8.7).

\section{Physical models}

Other methods, based on a more fundamental consideration of the origin of the collimator scatter components, have been suggested. By using Monte Carlo calculations, the head of the treatment unit can be modelled, from which the relative importance of the different components of $S_{c}$ can be deduced $[2,3,11]$. Using this method, the flattening filter and the primary collimator were clearly shown to be the most important contributors to $S_{c}[3,11]$. Ahnesjö et al. [3] developed a convolution method to describe the field size dependence of the output factors for use in a treatment planning system. Yu and Sloboda [74] applied an integration technique over the surface of the flattening filter as seen from the detector to predict $S_{c}$ in a two-component $x$-ray source model. Some empirical methods based on the form of the flattening filter and its relative position in the treatment head were discussed by Lam et al. [40]. A simple equivalent square formula was proposed by Kim et al. [35], in which a field that is defined in the source plane is mapped back into the detector plane by an equivalent field relationship. All these approaches have in common that geometrical details of the treatment head, sometimes deduced from measurements, are used in the models. Some have been developed as treatment planning system algorithms [3], others can be used in hand calculations [35]. Another simple and accurate method of calculating $S_{c}$ of a rectangular field was suggested by van Gasteren et al. [67]. In that paper, the amount of scatter is taken into account that arrives at the reference point from the beam flattening filter in the head of the treatment machine. When the scattering filter is viewed from this point, it is evident that for a square field $(X, X)$ the lower set of collimator blocks defines a larger part of the flattening filter than the upper set. A scaling term can be used to find the effective field size that defines a square projection at the level of the flattening filter when viewed from the reference point. This approach is described in somewhat more detail in Appendix 8.7.2, including the iteration steps needed to obtain the scaling factor. See also reference [67] for open beams and reference [68] for wedged beams.

If any of these approaches to obtain $S_{c}$ values for the full 2-D table of all rectangular field sizes used in the clinic is not possible, direct measurement is recommended instead. 


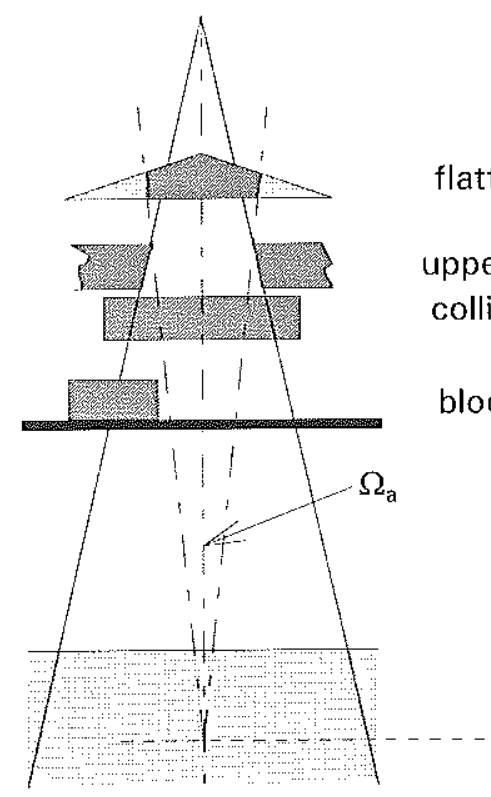

a)

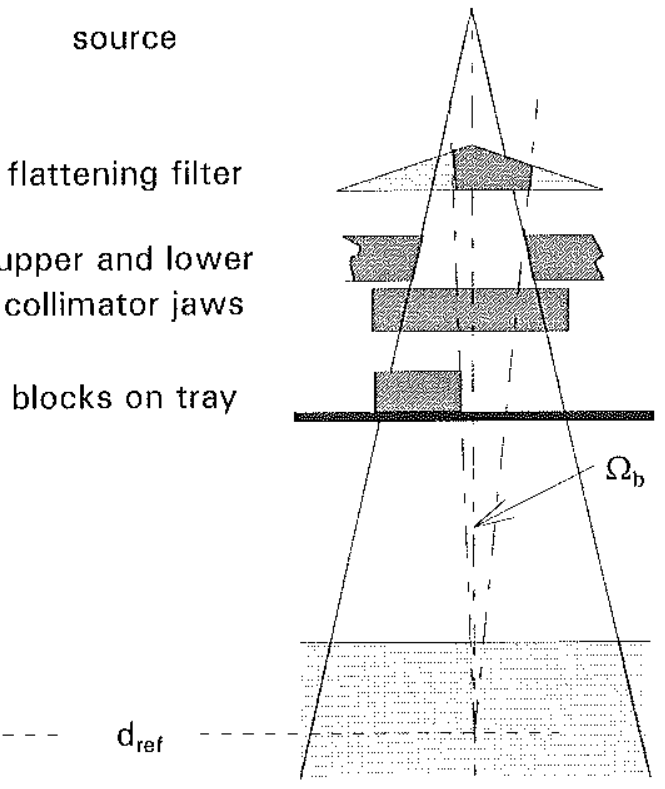

b)

Figure 3.1 Two situations with blocks on the tray are shown: a) with an open 'view' of the flattening filter resulting in a solid angle $\Omega_{\mathrm{a}} ;$ b) with the 'view' of the flattening filter partly shielded by a block, resulting in a smaller solid angle $\Omega_{\mathrm{b}}$.

The effect of partial shielding of the flattening filter on $S_{c}$

It has been shown that the amount of photons scattered in the treatment head and directed to the point of dose measurement is almost completely determined by the scattering processes in the flattening filter and the primary collimator. Photon scattering at the adjustable secondary collimator jaws can be ignored or is of minor importance $[2,11,44,60]$. The variation of the collimator scatter correction factor with changing collimator setting can, therefore, be related directly to the volume of the flattening filter 'seen' from the point of interest. Positioning of customized blocks on a tray below the collimator jaws might influence $S_{c}$ of the open beam due to the partial obscurance of the flattening filter as seen from the point of interest (see the solid angles $\Omega_{\mathrm{a}}$ and $\Omega_{\mathrm{b}}$ in figure 3.1). A general quantitative description of this effect on $S_{c}$ is difficult, but a geometrical examination of the construction of the head of various treatment machines shows that only in the case of rather extreme blocking the effect of the additional customized blocks is more important than the shielding effect of the movable collimator jaws $[41,45,60,63]$. The point where customized blocks will start to influence the $S_{c}$ value of the open field can be estimated (see Appendix 8.5) and depends on the construction details of the head of the treatment machine. These may differ considerably between the different types, 
as shown in figure 3.2. A detailed description of the head is needed for a proper application of the method suggested in Appendix 8.5.

The value of the collimator scatter correction factor of the blocked irradiation field, $S_{c, b l o c k}$ can thus be taken equal to $S_{c}$ of the unshielded beam, as long as the part of the flattening filter that can be seen from the point of measurement for the blocked field is determined by the collimator jaws only (figure 3.1.a). In other situations, $S_{c, b l o c k}$ must be determined by measurement and, consequently, must replace $S_{c}$ in the equations of chapter 2 (figure 3.1 b).

\section{The phantom scatter correction factor $S_{p}$}

For rectangular fields, changes in the energy spectrum of the primary photon beam with varying field size can be neglected. $S_{p}$ data for these fields can be obtained by applying Clarkson's method in a simple computer program using $S_{p}$ data determined for square fields [13]. The equivalent square fields method $[8,9,50]$ for the determination of the PDD and phantom scatter factors for nonsquare fields is widely used in clinical practice. Its accuracy is, however, somewhat inferior to that of the Clarkson method if the $S_{p}$ values have to be determined for different beam qualities. The equivalent field sizes given in the BJR Supplements 17 and 25 [8,9] are mean values obtained by considering photon beams over a wide range of radiation qualities, and, furthermore, in the determination of the equivalent square fields, the head and phantom scatter dose contributions were not separated.

By carrying out this separation and applying Clarkson's method for each beam quality independently, improvements of the order of magnitude of 0.5 to $1.0 \%$ are possible in the determination of $S_{p}$ especially for elongated fields. A new table of equivalent squares, to be used for $S_{p}$ (as well as for PDD and TPR), has been proposed for this purpose by Venselaar et al. [70]. It is based on data gathered from a large number of beam qualities in the range of ${ }^{60} \mathrm{Co}$ to $25 \mathrm{MV}$ and is included in this report (Appendix 8.6).

For irregular fields, neither the presence of shielding blocks nor the tray significantly influence the energy spectrum of the photon beam, and their phantom scatter correction factor $S_{p}\left(v_{p, \text { block }}\right)$ can be calculated from $S_{p}$ data for square fields, again applying Clarkson's method. 
3.

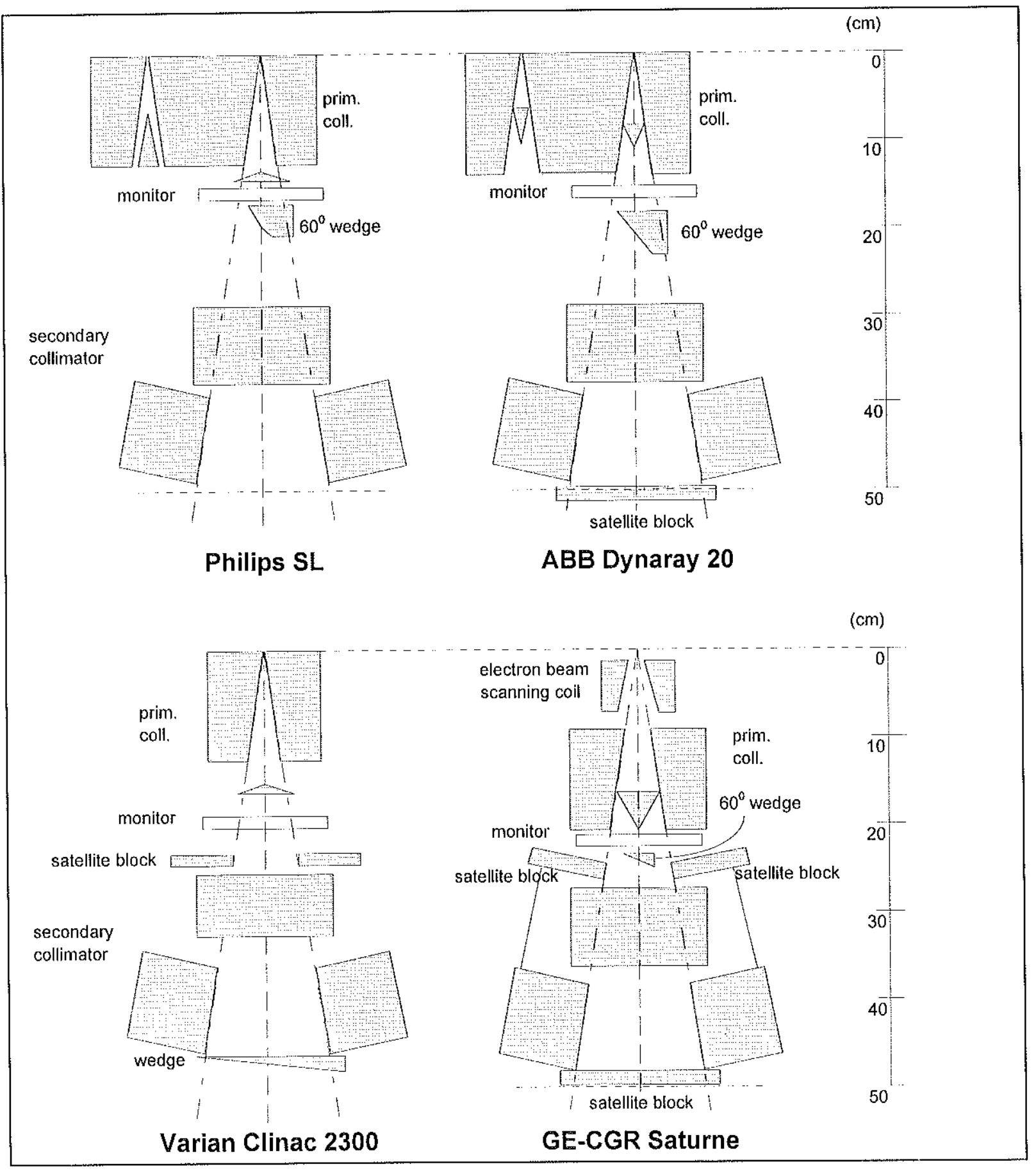

Figure 3.2 Construction drawings of the head of four different treatment machines. Note that the distance between the flattening filter and the upper collimator blocks differs considerably between the collimator designs. The geometry in which extreme blocking will influence $S_{c}$ will be different for the four machines. 


\subsection{Wedges and compensators}

Wedges and/or compensators are inserted in the beam to modify the dose distribution in the patient, e.g., to compensate for variations in body contour, for the presence of inhomogeneities or to generate intensity modulated beams that can be used to optimize the dose distribution. For these irradiation geometries $S_{c}$ values deviate from the values of the open beam due to changes in the energy fluence at the point of interest as well as in the amount of radiation scattered backwards into the monitor chamber in the head of the treatment machine $[22,26]$. Wedges and compensators are generally made of high- $Z$ materials. Depending on the construction of the treatment machine, wedges can be situated below the collimator jaws, or mounted in the head of the treatment machine between the monitor chamber and the collimator jaws. Compensators are, in general, placed below the collimator jaws. Both devices must be considered as an important additional extended source of scattered photons. These additional scattered photons in the beam can be taken into account by applying an effective source-surface distance in the inverse square law term in the dose calculation formalism instead of the geometrical distance as used for the open beam situation. Furthermore, the energy spectrum of the primary beam will be modified $[20,22,26,52,61]$.

In principle, both the wedge and the compensator influence the energy fluence per monitor unit at the point of interest in a similar way. Therefore, only the effect of the wedge will be discussed here.

Definition of the reference wedge transmission factor, $W F_{\text {ref }}$

For the open beam situation of an arbitrarily defined field, equation (2.3.3) is written as:

$$
\begin{aligned}
D_{\text {open }}\left(v_{c}, v_{p, f}, d, f\right) & =D_{\text {ref,open }} \times S_{c, \text { open }}\left(v_{c}\right) \times S_{p, o p e n}\left(v_{p, f} / F\right) \\
\times & \left\{\left(f_{\text {ref }}+d\right) /(f+d)\right\}^{2} \times R D D_{\text {open }}\left(v_{p, f} / F, d, f_{\text {ref }}\right)
\end{aligned}
$$

while for the same beam with a wedge the corresponding equation is:

$$
\begin{aligned}
D_{w}\left(v_{c}, v_{p, f}, d, f\right) & =D_{r e f, w} \times S_{c, w}\left(v_{c}\right) \times S_{p, w}\left(v_{p, f} / F\right) \\
& \times\left\{\left(f_{r e t, w}+d\right) /\left(f_{w}+d\right)\right\}^{2} \times R D_{w}\left(v_{p, f} / F, d, f_{r e f}\right)
\end{aligned}
$$

The subscripts of the quantities refer to the open and wedged beam situations, respectively. Note that in the wedged case the subscript $w$ is added to the source-surface distance, $f_{w}$ in the inverse square law term to indicate that an effective source-surface distance is used. 
3.

The wedge transmission factor WF is defined as the ratio of the dose per monitor unit measured with and without the wedge in the beam. It is emphasized that, by convention, this definition is given here only for the reference depth, $d_{r e f}$. This limitation is in agreement with the definitions of other quantities at the same depth (e.g. $S_{p}, S_{c}, D_{r e f}$ ), and with the practical use of wedge transmission factors. At the same time, it is concluded from this definition that the possible depth dependence of the wedge transmission factor is taken into account via the different RDDs of the wedged and open beam. These should be made available as separate measurements for the open and wedged beams. Thus:

$$
W F\left(v_{c}, v_{p}, d_{\text {ref }}, f\right)=D_{w}\left(v_{c}, v_{p}, d_{\text {ref } f} f\right) / D_{\text {open }}\left(v_{c}, v_{p}, d_{r e f} f\right)
$$

The wedge transmission factor WF is used as a measure of the wedge-induced change in the energy fluence per monitor unit measured at the point of interest (at the reference depth) in the beam [29]. Considering the arguments given above, the influence of the wedge varies with collimator setting $v_{c}$ and with source-surface distance. We now define $W F_{r e f}$, the reference wedge transmission factor, as the wedge transmission factor for the reference irradiation condition:

$$
\begin{aligned}
W F_{\text {ref }} & =W F\left(v_{\text {cref }}, v_{\text {pref }}, d_{\text {ref }}, f_{\text {ref }}\right) \\
& =D_{w}\left(v_{\text {cref }}, v_{\text {pref }}, d_{\text {ref }}, f_{\text {ref, } w}\right) / D_{\text {open }}\left(v_{\text {cref }}, v_{\text {pref }}, d_{\text {ref }}, f_{\text {ref }}\right) \\
& =D_{\text {ref }, w} / D_{\text {ref,open }}
\end{aligned}
$$

Then, by combining $(3.2 .1)$ to $(3.2 .4)$ it is found that

$$
\begin{aligned}
W F\left(v_{c}, v_{p}, d_{r e f}, f\right)= & W F_{r e f} \times\left(S_{c, w}\left(v_{c}\right) / S_{c, o p e n}\left(v_{c}\right)\right) \\
& \times\left(S_{p, w}\left(v_{p, f} / F\right) / S_{p, o p e n}\left(v_{p, f} / F\right)\right) \\
& \times\left\{\left(f_{r e f, w}+d_{r e f}\right) /\left(f_{w}+d_{r e f}\right)\right\}^{2} /\left\{\left(f_{r e f}+d_{r e f}\right) /\left(f+d_{r e f}\right)\right\}^{2}
\end{aligned}
$$

In order to simplify this expression, the factors are discussed separately.

\section{$W F_{\text {ref }}$}

Wedge induced changes in the energy spectrum of the scattered beam are larger for low energy than for high energy megavoltage photon beams. It was shown that for a $4 \mathrm{MV} x$-ray beam of an $A B B$ linear accelerator this effect results in a $1.5 \%$ lower value of the wedge factor if measured in a full scatter phantom compared with values determined in a mini-phantom, independent of field size [22]. For this reason the general rule should be that the reference wedge transmission factor is determined in a full scatter phantom. 
The ratio $S_{c, w} / S_{c, o p e n}$

Because the wedge behaves as an extended source of scattered photons, a stronger variation of the energy fluence per monitor unit at the point of interest with collimator setting will occur. For example, $S_{c, w}\left(v_{c}\right)$ varies with the collimator setting over a wider range than $S_{c, \text { open }}\left(V_{c}\right)$ for the open beams. Variations up to $8 \%$ have been reported $[22,61,75]$. If the wedge is situated at a fixed position in the treatment head the magnitude of this effect will be less pronounced if it is measured at larger wedge-to-point distances, i.e. at larger SSDs. This is explained by the fact that the contribution to the energy fluence per monitor unit at the point of interest of the photons coming from the wedge, relative to the contribution of the primary and scattered photons originating from the flattening filter, will decrease for larger SSDs because of the different relative positions of the wedge and the target with respect to the point of interest [22].

\section{The ratio $S_{p, w} / S_{p, o p e n}$}

For most photon beam qualities, no change in $S_{p}$ has been observed if a wedge is inserted in a photon beam [22]. As the shift in the quality index of a wedged beam is relatively small compared with the open beam situation, this result is in agreement with the observed slow variation of $S_{p}$ with beam quality (see also section 5.3.1) [56]. Consequently, the ratio of $S_{p}$ values in wedged and open beams, as required in equation $(3.2 .5)$, is close to unity. For the purpose of calculation of monitor units, this means that $S_{p}$ values of the open beam can also be applied for the wedged beam.

\section{The ratio of inverse square law terms}

The main source of photons in an open beam is a small focal spot on the target of the linear accelerator. If a wedge is inserted, a diffuse source of scattered photons is added to the beam. The angular distribution of the scattered photons from a wedge is relatively wide. If the wedge is positioned high up in the beam defining system of the machine, the extra photon contribution may still be assumed to obey the inverse square law at clinically used distances. The effective source-surface distance is then close to that of the open beam. Nevertheless, small deviations may occur and these should be taken into account in dose calculations. The magnitude of the deviations has to be determined for each beam separately, by performing measurements of wedge transmission factors at different source-surface distances. If a wedge is positioned below the collimator jaws (see figure 3.2 for an example), the effect is more pronounced because of the shorter distance between wedge and point of measurement.

\section{Influence of a wedge on RDD (PDD)}

The above mentioned wedge induced change in the energy spectrum of the primary photon beam can result in a different RDD (PDD) curve compared to the open beam. This variation is usually attributed to beam hardening, although beam softening might also occur, especially for high energy photon beams 
3.

[22,52]. In addition, wedge-induced changes in the electron contamination can drastically change the RDD (PDD) curve in the first few centimeters. Therefore, for treatment planning purposes, it is strongly recommended to measure and implement depth dose curves for wedged photon beams, $\mathrm{RDD}_{\mathrm{w}}$.

\section{Definition of the relative wedge transmission factor, $R W F$}

We can now define the relative wedge transmission factor, $R W F\left(v_{c}, f\right)$, as the ratio of the wedge transmission factor at depth $d_{r e f}$ in a particular irradiation condition $W F\left(v_{c}, v_{p}, d_{r e f} f\right)$, and the reference wedge transmission factor $W F_{\text {ref }}$ $R W F\left(v_{c}, f\right)=W F\left(v_{c}, v_{p}, d_{r e f} f\right) / W F_{r e f}$. Using the assumption, as described above, that the ratio of phantom scatter correction factors is close to unity, RWF is then found from equation (3.2.5) as,

$$
\operatorname{RWF}\left(v_{c}, f\right)=S_{c, w}\left(v_{c}\right) / S_{c, o p e n}\left(v_{c}\right) \times\left\{\left(f_{r e f, w}+d_{r e f}\right) /\left(f_{w}+d_{r e f}\right)\right\}^{2} /\left\{\left(f_{\text {ref }}+d_{\text {ref }}\right) /\left(f+d_{\text {ref }}\right)\right\}^{2}
$$

Combining the equations now yields the dose in a wedged beam, which can be calculated according to:

$$
\begin{aligned}
D_{w}\left(v_{c}, v_{p, f}, d, f\right)= & D_{o p e n}\left(v_{c}, v_{p, f}, d, f\right) \times W F_{r e f} \times R W F\left(v_{c}, f\right) \\
& \times R^{R D D_{w}}\left(v_{p, f} / F, d, f_{\text {ref }}\right) / R D_{\text {open }}\left(v_{p, f} / F, d, f_{r e f}\right)
\end{aligned}
$$

It should be noted here that expression (3.2.6) is only meant to show which factors influence the value of RWF. It is not meant to derive RWF values from experimentally determined effective source-surface distances. The most straightforward approach is to assume $f_{w}$ to be equal to $f$ and to determine RWF by experiment as a function of the collimator setting $v_{c}$ for a number of different SSDs. From these measurements, interpolation and extrapolation will yield a good result for most clinical situations. $R W F\left(v_{c}, f\right)$ can be determined in a full scatter phantom or in a mini-phantom. The measurement geometries are shown in figure 3.3 .

In summary, the factor $W F_{\text {ref }}$ takes into account the reduction of the energy fluence per monitor unit at the point of interest with respect to the open beam for the reference irradiation condition. The factor RWF takes into account the variation in this reduction for non-reference field sizes and non-reference SSDs.

\section{Influence of field elongation}

The measurement of wedge transmission factors is often limited to square fields in the range of field sizes used clinically. Several authors presented measurements of wedge factors in rectangular fields $[52,61,68]$. The best approach appears to apply an equivalent square field size method and then to 
3.

interpolate between measured values of square fields, as long as the elongation ratio of the fields is less than 3 . For ratios larger than 3 , it is recommended to determine the wedge transmission factors by measurement. In this way, an agreement between prediction and measurement in rectangular wedged fields within $1 \%$ can be achieved $[52,58,62]$.

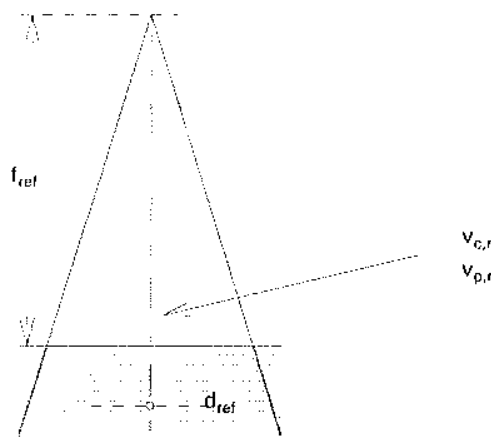

a) $D_{\text {tel }}$

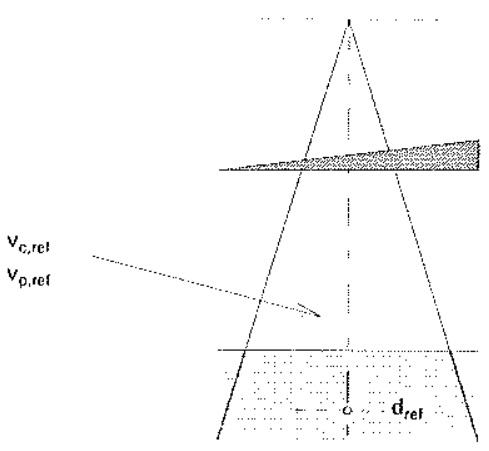

b) $\mathrm{D}_{t \mathrm{ef}} \mathrm{WF}$

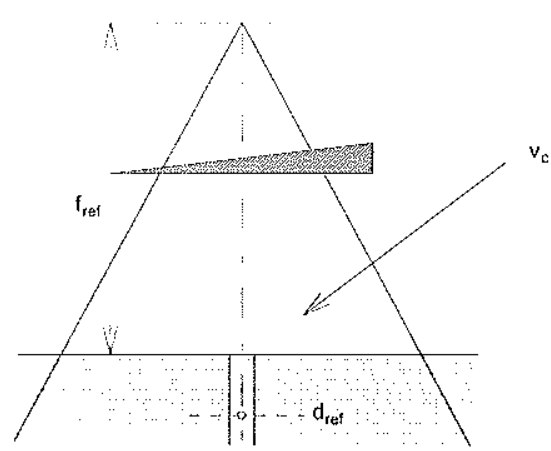

c) $D_{\text {ref }} W F_{\text {ref }} R W F\left(v_{c}, f_{\text {ref }}\right) S_{c}\left(v_{c}\right) S_{p}\left(v_{p}\right)$

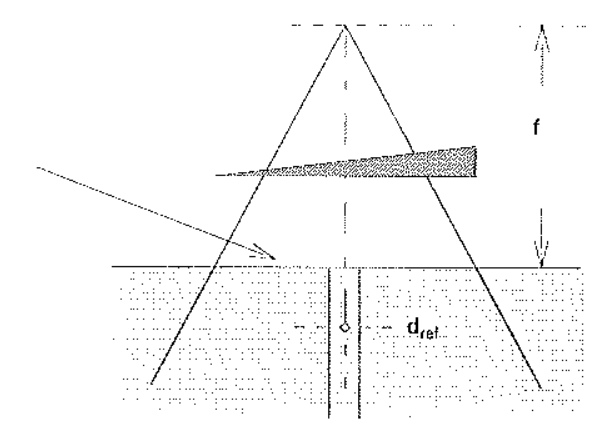

d) $D_{\text {ret }} W F_{\text {rel }} R W F\left(V_{c_{r}} f\right) S_{c}\left(v_{c}\right) S_{\rho}\left(v_{\rho f} / F\right)\left\{\left(f_{r e f}+d_{r e f}\right\} /\left\{f+d_{r e f}\right\}\right\}^{2}$

Figure 3.3 $W F_{\text {ref }}$ is derived from measurements a) and b) in a full scatter phantom. For the reference source-surface distance $f_{\text {ref }}, R W F\left(v_{c}, f_{\text {ret }}\right)$ is found as a function of $v_{c}$ from the ionization chamber readings in the full scatter phantom or in a mini-phantom, resulting from measurements b) and cl, with and without a wedge in the beam. To investigate the influence of the sourcesurface distance on the value of RWF $\left(v_{c}, f\right)$, measurements $\left.c\right)$ and d) at different SSDs, but with the same collimator setting, should be performed. 
3.

\section{Compensators}

A compensator can be considered as a more sophisticated type of wedge, influencing the dose distribution in any direction perpendicular to the beam axis, instead of the one-directional effect typical of a wedge. Compensators are generally customized for the individual patient and, therefore, published data are scarce. However, it can be expected that the influence of the compensator on the phantom scatter shows the same behaviour as that observed with a wedge. Only wedge induced effects are discussed here for this reason. In case compensators are used, the same recommendations apply, which should be verified for a few specific situations. 


\subsection{Fields with a tray}

In clinical practice, irradiation fields are often partially shielded with customized blocks from high- $Z$ material positioned on a tray which, in turn, is mounted below the collimator jaws of the treatment machine. The source-to-tray distance is approximately $2 / 3$ of the SAD. For a clear understanding of the resulting changes in collimator scatter correction factors it is helpful to separate the origin of the changes of $S_{c}$ into two parts: the influence of photon attenuation and scatter in the material of the block support, or tray, on the energy fluence at the point of interest, and the energy fluence reduction due to the partial shielding of the flattening filter by the additional customized blocks. This latter effect has already been discussed in section 3.1.

\section{The effect of a tray on the dose}

The tray on which the blocks are positioned attenuates the energy fluence of the primary photons in the beam. In addition, scattering of photons in the tray material takes place, contributing to the dose at the point of interest [30]. The relative importance of this effect depends on the beam quality, the thickness and material of the tray, the actual size of the irradiated tray surface, and the distance between the tray and the phantom or patient surface [21]. The presence of the tray in the photon beam will not result in an appreciable amount of backscattered photons into the monitor chamber, due to their large mutual distance. However, the effects on the dose distribution due to the presence of a tray in a beam are essentially the same as for wedges and compensators.

The effect of the tray on the dose value at a particular point is usually taken into account by applying a tray factor. This tray transmission factor TF is defined as the ratio of the dose measured at the reference depth, with and without the tray in the beam for the same number of monitor units:

$$
T F\left(v_{c}, v_{p}, d_{r e f}, f\right)=D_{\text {tray }}\left(v_{c}, v_{p}, d_{r e f}, f\right) / D_{\text {open }}\left(v_{c}, v_{p}, d_{r e f}, f\right)
$$

Two comments with respect to the tray transmission factor can be made. Firstly, for a particular quality of the photon beam and tray design, the influence of the tray on the energy fluence varies with the actual field size projected on the tray, $v_{\text {tray, }}$ taking the blocked area into account. The magnitude of this variation depends on the tray-phantom distance TPD and therefore on the source-surface distance, since the tray is mounted at a fixed position below the head of the treatment machine. In a similar way as for the wedge transmission factor, TF can be written as the product of the reference tray transmission factor $\mathrm{TF}_{\mathrm{ref}}$ and a relative tray factor $\mathrm{RTF}\left(\mathrm{v}_{\text {tray }}, \mathrm{TPD}\right)$, which takes into account the field size and distance dependence. The reference tray transmission factor $\mathrm{TF}_{\mathrm{ref}}$ is the transmission factor measured in the reference irradiation set-up: 


$$
\begin{aligned}
T F_{\text {ref }} & =T F\left(v_{\text {cref }}, v_{\text {pref }}, \mathrm{d}_{\text {ref }}, f_{\text {ref }}\right) \\
& =D_{\text {tray }}\left(v_{\text {cref }}, v_{\text {pref }}, \mathrm{d}_{\text {ref }}, f_{\text {ref }}\right) / D_{\text {open }}\left(v_{\text {cref }}, v_{\text {pref }}, d_{\text {ref }}, f_{\text {ref }}\right)
\end{aligned}
$$

The relative tray transmission factor RTF( $v_{\text {tray }}$ TPD) can be found from the ratio of $T F\left(v_{\text {tray }}, T P D\right)$ and $T F_{\text {ref, }}$ which is equivalent to the ratio of collimator scatter correction factors measured with and without a tray in the beam:

$$
R T F\left(v_{\text {tray }}, T P D\right)=\operatorname{TF}\left(v_{\text {tray }}, T P D\right) / T_{\text {ref }}=S_{c, \text { tray }}\left(v_{c}\right) / S_{c, o p e n}\left(v_{c}\right)(3.3 .3)
$$

As discussed previously for wedges, the presence of the tray has only a minor influence on the phantom scatter contribution and, therefore, on the percentage depth dose value at depth $d_{\text {ref }}[22]$.

The second comment about the tray factor is that RTF( $v_{\text {tray, }}$ TPD $)$ is close to unity when nearly water equivalent materials, e.g. PMMA, with thicknesses up to 10 $\mathrm{mm}$ are applied: RTF varies less than $2 \%$ for field sizes varying from $4 \mathrm{~cm} \times 4$ $\mathrm{cm}$ to $40 \mathrm{~cm} \times 40 \mathrm{~cm}$ [69]. A more pronounced variation will be observed for larger thicknesses of the tray material and/or shorter TPDs. Because of the relatively small effects found experimentally for PMMA thicknesses $\leq 10 \mathrm{~mm}$, one may in general replace $v_{\text {tray }}$ by the collimator setting $V_{c}$, unless extreme blocking is applied: $R T F\left(v_{\text {tray }}, T P D\right)=R T F\left(v_{c}, T P D\right)$.

From these observations it can be concluded that the tray transmission factor can be measured with the mini-phantom as well as in a full scatter phantom, according to the measurement geometries of figure 3.4. The values of RTF $\left(v_{\text {tray }}\right.$, TPD $)$ have to be obtained for a number of square field sizes covering the full range of clinically used fields and a number of tray-phantom distances (see figure 3.4). In a similar way as for wedges, it is recommended to repeat the measurements at two other source-surface distances, for example 80 and 120 $\mathrm{cm}$. For rectangular fields, an equivalent square field method can be used to obtain a reliable interpolation.

\section{Influence of a tray on RDD}

It has to be noted that for short distances between the tray and phantom surface, electron contamination will substantially increase the surface dose. This effect will not influence the readings resulting from the choice of $d_{\text {ref }}$ at a depth larger than the range of the contaminating electrons. The variation in the amount of contaminating electrons, however, will affect the RDD of the open beam in the first few centimeters. If clinically relevant, these deviations could be determined by measurements and incorporated in a $\mathrm{RDD}_{\text {block }}$. 
3.

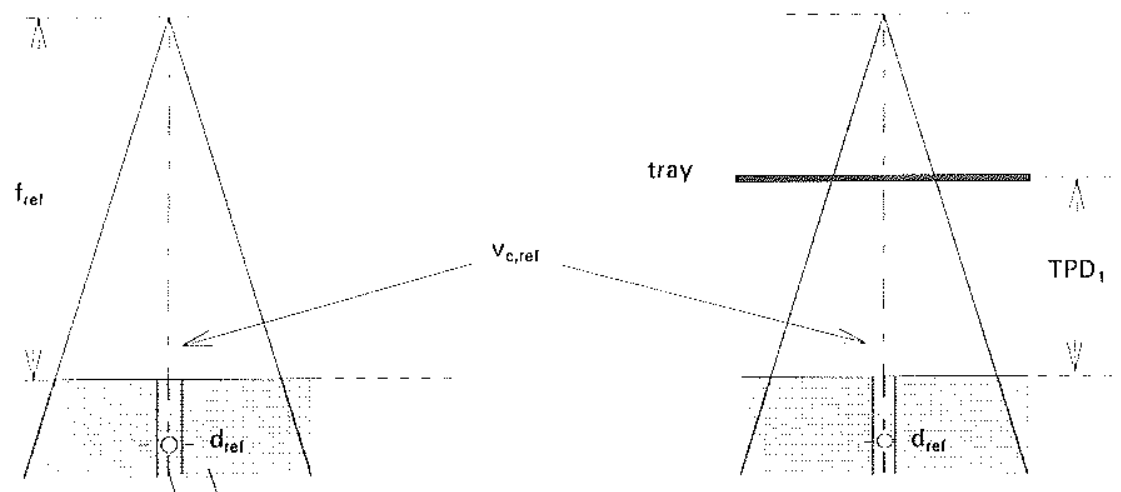

a) $D_{\text {ief }}$

b) $D_{\text {ret }} T F_{\text {ret }}$

full-scatter phantom

mini-phantom

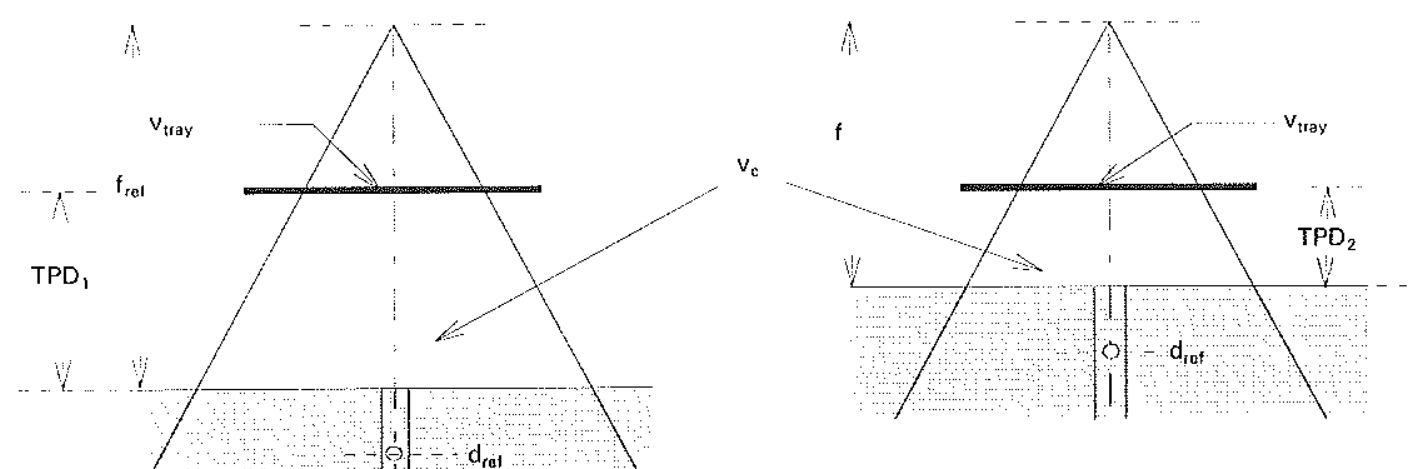

c) $D_{\text {ret }} T F_{\text {ief }} R T F\left(v_{\text {tray }} T P D_{1}\right) S_{c}\left(v_{c}\right) S_{p}\left(v_{p}\right)$

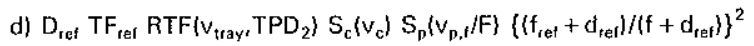

Figure 3.4 The reference tray transmission factor $T F_{r e f}$ is derived from measurements a) and b); then, for a specified tray-phantom distance TPD, the relative tray factor RTF $\left(V_{\text {tray }}, T P D\right)$ can be derived from $b$ ) and $c$ ) as a function of $v_{\text {tray }}$. For a specified $v_{t r a y}$ RTF $\left(v_{\text {tray }}\right.$,TPD $)$ can be derived from measurements $c$ ) and d) at other SSDs. Measurements can be performed both in the miniphantom and in the full scatter phantom. In most cases, $v_{\text {tray }}$ can be replaced by $v_{c}$ (see text). 


\subsection{Recommendations with respect to monitor unit calculations}

\section{Wedges and compensators}

It is recommended to determine by measurement the wedge transmission factor $W F_{\text {ref }}$ in the reference irradiation set-up. The relative wedge factor $R W F\left(v_{c}, f\right)$ has to be measured as a function of the collimator setting $v_{c}$, i.e. for the full range of square fields. This measurement of RWF has to be repeated for a number of source-surface distances $f$, covering the range of SSDs applied in clinical routine. Furthermore, the relative depth dose curves of the wedged photon beam, $\operatorname{RDD}_{w}\left(v_{p}, d, f\right)$, have to be measured.

In the calculation of the number of monitor units $N$, equations (2.4.1) and (2.4.2) must be modified to take the presence of the wedge into account. The product of $S_{c}\left(v_{c}\right) \times R D D\left(v_{p}, d, f\right)$ of the open field is replaced by the product of $S_{c}\left(v_{c}\right) \times W F_{r e f} \times R W F\left(v_{c}, f\right) \times \operatorname{RDD}_{w}\left(v_{p}, d, f\right)$, which leads for beams with a sourcesurface distance $=f_{\text {ref }}$ to:

$$
\begin{aligned}
N=D_{\text {presc }} /\left\{D_{\text {cal }} \times W F_{\text {ref }} \times R W F\left(v_{c}, f\right) \times S_{c}\left(v_{c}\right) \times S_{p}\left(v_{p}\right)\right. \\
\left.\times R D D_{w}\left(v_{p}, d, f_{r e f}\right) / R D D_{\text {open }}\left(v_{p, \text { ref }}, d_{m}, f_{r e f}\right)\right\}(3.4 .1)
\end{aligned}
$$

and for beams with source-surface distance $\neq f_{\text {ref }}$ to:

$$
\begin{aligned}
N= & D_{\text {presc }} /\left\{D_{\text {cal }} \times W F_{\text {ref }} \times R W F\left(v_{c}, f\right) \times S_{c}\left(v_{c}\right) \times S_{p}\left(v_{p, f} / F\right)\right. \\
& \left.\times\left\{\left(f_{\text {ref }}+d\right) /(f+d)\right\}^{2} \times R D D_{w}\left(v_{p, f} / F, d, f_{r e f}\right) / R D D_{o p e n}\left(v_{p, r o f}, d_{m}, f_{r e f}\right)\right\}
\end{aligned}
$$

\section{Fields with tray and blocks}

It is recommended to determine the tray transmission factor $\mathrm{TF}_{\text {ref }}$ in the reference irradiation set-up. Also, the relative tray factor RTF( $v_{\text {tray }}$, TPD) has to be determined by measurement of the full range of square field sizes defined at the level of the tray, $v_{\text {tray }}$ and for a few representative tray-phantom distances, TPD. Tray transmission factors may be measured with the mini-phantom as well as in a full scatter phantom. In addition, $S_{c, b l o c k}$ has to be determined by the measurement of irradiation geometries where extreme blocking is applied.

In the calculation of the number of monitor units, equations (2.4.1) and (2.4.2) must be modified to take into account the effect of the tray and of the shielding blocks. The factor $S_{c}\left(V_{c}\right)$ for the open fields is replaced by the product of $T F_{r e f} x$ $R T F\left(v_{\text {tray }}, T P D\right) \times S_{c, b l o c k}\left(v_{\text {tray }}\right)$, which leads for beams with source-surface distance $f_{\text {ref }}$ to: 
3.

$$
\begin{aligned}
N=D_{\text {presc }} /\left\{D_{\text {cal }} \times\right. & T_{\text {ref }} \times R T F\left(v_{\text {tray }}, T P D\right) \times S_{c, b l o c k}\left(v_{\text {tray }}\right) \times S_{p}\left(v_{p}\right) \\
& \left.\times \operatorname{RDD}_{\text {block }}\left(v_{p}, d, f_{\text {ref }}\right) / \operatorname{RDD}\left(v_{p, \text { ret }}, d_{m}, f_{\text {ref }}\right)\right\}
\end{aligned}
$$

and for beams with source-surface distance $\neq f_{\text {ref }}$ to:

$$
\begin{aligned}
N= & D_{\text {presc }} /\left\{D_{\text {cal }} \times T F_{\text {ref }} \times R T F\left(v_{\text {tray }} T P D\right) \times S_{c, \text { block }}\left(v_{\text {tray }}\right) \times S_{p}\left(v_{p, f} / F\right)\right. \\
& \left.\times\left\{\left(f_{\text {ref }}+d\right) /(f+d)\right\}^{2} \times R D D_{\text {block }}\left(v_{p, f} / F, d, f_{\text {ref }}\right) / R D D\left(v_{p, r e f}, d_{m}, f_{\text {ref }}\right)\right\}
\end{aligned}
$$

Note 1: for compensators, the recommendation is similar to that for wedged fields.

Note 2: if combinations of wedges and blocked fields are used, both correction steps have to be introduced in the calculation procedures. 


\section{Experimental determination of scatter correction factors}

\subsection{Square and rectangular fields}

$S_{c}\left(v_{c}\right)$ has to be measured for each available photon beam quality. The ionization chamber is placed in the mini-phantom on the central axis of the beam, with the surface of the phantom positioned at an SSD equal to the SAD (figure 2.1). The chamber axis may be parallel to the axis of the beam to allow measurements of small fields (see also Appendix 8.8). The ionization chamber readings have to be corrected for stem and/or cable effects, if present. The construction of the miniphantom is such that the centre of the air cavity of the chamber, assumed to be the effective point of measurement, is positioned at a depth of $10 \mathrm{~cm}$. Note that the actual position of the effective point of measurement is not critical. It is, however, essential that the measurements are restricted to field sizes larger than the size of the mini-phantom itself, to avoid any penumbra effect and/or field size dependent phantom scatter dose contribution in the measurement of $S_{c}$.

$S_{c}\left(v_{c}\right)$ has to be determined for square and rectangular collimator settings corresponding to those generally used in the clinic, from a minimum field size of $4 \mathrm{~cm} \times 4 \mathrm{~cm}$ or smaller if possible, up to the maximum field size, e.g. $40 \mathrm{~cm} \times$ $40 \mathrm{~cm}$. In order to obtain $S_{c}$ values for the rectangular fields, two approaches can be followed. In the first approach, $S_{c}$ is simply measured for the full range of rectangular collimator settings, with the $\mathrm{X}$ - and $\mathrm{Y}$-jaws set independently at, for example, $3,4,5,6,8,10,12,15,20,25,30,35$ and $40 \mathrm{~cm}$. In the second approach, a limited set of square and rectangular fields could be measured, after which a data fitting method must be applied to complete the 2-D table of $S_{c}$ values. For this purpose, a method based on a physical model of the beam is suggested by van Gasteren et al. [67], while analytical methods were discussed by Jager et al. [31]. The proposed approach of Jager et al. is based on a set of measurements of eight square and twelve rectangular fields. For both approaches and for references to other methods, more details are given in Appendix 8.7.2.

$\mathrm{S}_{\mathrm{cp}}\left(\mathrm{v}_{\mathrm{c}}, \mathrm{v}_{\mathrm{p}}\right)$ has to be measured for each available beam quality using the geometry shown in figure 2.1. An ionization chamber, with its effective point of measurement at depth $d_{\text {ref }}=10 \mathrm{~cm}$ on the beam axis, is placed in a full scatter water phantom. The phantom surface is set at an SSD equal to the SAD, usually $100 \mathrm{~cm}$ for linear accelerators and $80 \mathrm{~cm}$ for ${ }^{60} \mathrm{Co}$ units. The reading of the ionization chamber should be corrected for stem and/or cable effects if present.

It is recommended to measure $S_{c p}$ data in the same square field size settings as used in the $S_{c}$ measurements. For the purpose of checking the results, it is recommended to obtain $S_{c p}$ values by direct measurement for a number of rectangular fields. 
Phantom scatter correction factors $S_{p}\left(v_{p}\right)$ for square fields can be derived from the ratio of $S_{c p}\left(v_{c}, v_{p}\right)$ and $S_{c}\left(v_{c}\right)$ by using expression (2.1.3). These results should be compared with data published in this report; see section 5.3 and Appendix 8.7.1. Deviations in $S_{p}$ values larger than $1 \%$ should be investigated.

The procedures used in the clinic to derive $S_{c}$ and $S_{p}$ values for rectangular fields, e.g. based on an equivalent squares method or on look-up tables, should be checked for consistency with the measurements. It is recommended to try and maintain the difference between the product of the $S_{c}$ and $S_{p}$ data, obtained in this way, and the $S_{c p}$ values from direct measurement within $1 \%$.

\subsection{Wedges and compensators}

For wedged fields the reference wedge transmission factor $W F_{\text {ref }}$ should be determined in a full scatter phantom, while the relative wedge transmission factor for other field sizes and source-surface distances, RWF $\left(v_{c}, f\right)$, may be determined in the full scatter phantom or in the mini-phantom (see figure 3.3). The use of higher density build-up caps, e.g. brass, instead of a mini-phantom for the measurement of RWF is not recommended $[23,73]$. WF ref is the ratio of the dose per monitor unit in the reference irradiation set-up with a wedge in the beam to the dose per monitor unit in the same field without the wedge: $W F_{\text {ref }}=$ $D_{w}\left(v_{c, r e f}, v_{p, r e f}, f_{r e f}\right) / D_{o p e n}\left(v_{c, r e f}, v_{p, r e f}, f_{r e f}\right)$. RWF is found from the ratio of the wedge transmission factors determined for a specified field size and SSD, normalized to unity for the reference geometry: $R W F\left(v_{c}, f\right)=W F / W F_{r e f}$.

The number of relative wedge transmission factor measurements can be limited to square fields in the range of field sizes used clinically. It has been shown that for rectangular wedged fields, the relative wedge transmission factor can be determined in a first approximation by applying the equivalent square field size method and interpolating between measured $S_{c}$ values of square fields $[52,58,61]$. This should be checked by direct measurements of a few elongated fields. As for the open beams, relative depth dose curves for wedged beams $\operatorname{RDD}_{w}\left(v_{p}, d, f_{r e f}\right)$ have to be determined for square field sizes.

Systematic determination of the influence of a compensator on $S_{c}, S_{p}$ and RDD is not easy to perform due to the variability in the $3-D$ geometrical characteristics of the compensator. Therefore, it is recommended to directly measure the effect of a number of compensators typically used in the clinic on the dose at the specification point. 


\subsection{Blocked fields}

For the determination of the reference tray transmission factor $T F_{\text {ref }}$ measurements must be performed in the reference irradiation set-up (with and without the tray in the beam) for the same number of monitor units. The field size dependence, accounted for in the relative tray transmission factor RTF, should be determined for a number of square field sizes covering the range of 4 $\mathrm{cm} \times 4 \mathrm{~cm}$ to $40 \mathrm{~cm} \times 40 \mathrm{~cm}$. The dependence of TF on the tray-phantom distance TPD is found by repeating this measurement at other source-surface distances, for example, at SSD equal to $80 \mathrm{~cm}$ and $120 \mathrm{~cm}$. RTF is found from the ratio of the tray transmission factors determined for a specified field size and tray-phantom distance, normalized to unity for the reference field: $R T F\left(V_{c}\right)=T F /$ $T_{\text {ref }}$. TF and RTF can be determined using either a mini-phantom or a full scatter water phantom. The measurement set-up is similar to that used for the determination of $S_{c}$ and $S_{c p}$ and is illustrated in figure 3.4.

If extreme blocking of fields is used, $S_{c, \text { block }}$ has to be determined by measurements using a mini-phantom. The measurements are only needed for situations where the flattening filter, as seen from the point of interest, is partially or totally shielded by the additional blocks. The most practical way to perform this measurement is to leave the tray in the beam in both situations and to remove the field size dependence of the tray by dividing the readings for the blocked fields by the relative tray factor RTF. The result of the division is $S_{c, \text { block }}$ which replaces $S_{c}$ of open fields in the mathematical expressions. For blocked fields it is not necessary to determine $S_{p}$ from direct measurements of $S_{c p}$ : It is sufficient to use the phantom scatter correction factor of a field $v_{p}$ equivalent to that of the blocked field size.

As a final check, it is recommended to check by direct measurement the calculated product $T F_{\text {ref }} \times R T F\left(v_{\text {tray, }} T P D\right) \times S_{c, \text { block }}\left(v_{\text {tray }}\right) \times S_{p}\left(v_{p}\right)$ for several blocked fields. Correspondence should be within $1 \%$. 


\subsection{Summary}

Table 4.1 Summary of measurements to obtain the data needed for dose calculation procedures.

\begin{tabular}{|c|c|c|c|c|c|}
\hline quantity & field description & square fields ${ }^{11}$ & rectangular fields & $S S D$ & phantom $^{21}$ \\
\hline RDD & $\begin{array}{l}\text { open } \\
\text { wedged }\end{array}$ & $\begin{array}{l}+ \\
+\end{array}$ & $\begin{array}{l}+31 \\
+{ }^{31}\end{array}$ & $\begin{array}{l}100 \\
100\end{array}$ & $\begin{array}{l}f s p \\
f s p\end{array}$ \\
\hline$S_{c p}$ & $\begin{array}{l}\text { open } \\
\text { wedged }\end{array}$ & $\begin{array}{l}+ \\
+\end{array}$ & $\begin{array}{l}+3) \\
+31\end{array}$ & $\begin{array}{l}100 \\
100\end{array}$ & $\begin{array}{l}f s p \\
f s p\end{array}$ \\
\hline$S_{c}$ & $\begin{array}{l}\text { open } \\
\text { wedged } \\
\text { tray }\end{array}$ & $\begin{array}{l}+ \\
+ \\
+\end{array}$ & $\begin{array}{l}+41 \\
+31 \\
\end{array}$ & $\begin{array}{l}100 \\
100 \\
100\end{array}$ & $\begin{array}{l}m p \\
m p \\
m p\end{array}$ \\
\hline $\begin{array}{l}\text { WF } \\
T_{r o f} \\
r \theta f\end{array}$ & $\begin{array}{l}\text { wedged/open } \\
\text { tray/open }\end{array}$ & $\begin{array}{l}10 \mathrm{~cm} \times 10 \mathrm{~cm} \\
10 \mathrm{~cm} \times 10 \mathrm{~cm}\end{array}$ & - & $\begin{array}{l}100 \\
100\end{array}$ & $\begin{array}{l}\text { fsp } \\
m p \text { or } f s p\end{array}$ \\
\hline $\begin{array}{l}\text { RWF } \\
\text { RTF }\end{array}$ & $\begin{array}{l}\text { wedged } \\
\text { tray }\end{array}$ & $\begin{array}{l}+ \\
+\end{array}$ & $\begin{array}{l}+{ }^{3)} \\
-\end{array}$ & $\begin{array}{l}80,100,120 \\
80,100,120\end{array}$ & $\begin{array}{l}m p \text { or } f s p \\
m p \text { or } f s p\end{array}$ \\
\hline
\end{tabular}

1. For these measurements the side of the square fields can, for example, be set to $3,4,5,6,8,10,12$, $15,20,25,30,35$ and $40 \mathrm{~cm}$.

2. $\mathrm{fsp}=$ full scatter phantom; $\mathrm{mp}=$ mini-phantom

3. For these sets of measurements a limited number of elongated fields has to be chosen for the purpose of checking the data against published data (e.g. $S_{p}$ ) and/or confirmation of the outcome of calculations; for example, the application of the equivalent square field method.

4. Full sets of rectangular fields are needed, with independent setting of the $X$-and $Y$-collimator jaw at, for example, $3,4,5,6,8,10,12,15,20,25,30,35$ and $40 \mathrm{~cm}$. Fitting procedures may be applied to limit the number of measurements of rectangular fields. 


\section{Experimental data}

$\mathrm{S}_{\mathrm{cp} r} \mathrm{~S}_{\mathrm{c}}$ and $\mathrm{S}_{\mathrm{p}}$ data have been collected for a wide range of photon beam qualities (from ${ }^{60} \mathrm{Co}$ to $25 \mathrm{MV}$ photon beams) and a variety of treatment machines, using the methods described in the previous sections. In table 5.1, some characteristics of these beams and machine types are listed. Nominal beam energies and quality indices have been tabulated. The last columns show whether data of square and rectangular fields or only square fields were available. The most typical observations will be briefly discussed in the following sections.

\subsection{Total scatter correction factors, $\mathrm{S}_{\mathrm{cp}}$}

\subsection{1 $\quad S_{c p}$ for square fields}

$\mathrm{S}_{\mathrm{cp}}$ values determined in a number of photon beams are shown in figures 5.1.c to 5.8.c as a function of the side of a square field. The data given in these figures indicate that beams with the same nominal energy, but generated by different machines, give different $S_{c p}$ values. Note that the $S_{c p}$ curves from the GE-CGR accelerator beams are much steeper than those from other machines with the same nominal beam energy. This effect was observed earlier by several other groups $[33,45,57]$ and is explained by the construction details of the treatment head (see figure 3.2). The flattening filter of GE-CGR machines is much wider, and is positioned at a more downstream position compared with other machines.

\subsection{Collimator scatter correction factors, $\mathrm{S}_{\mathrm{c}}$}

\subsection{1 $\quad S_{c}$ for square fields}

$S_{c}$ values of the same beams are shown in figures 5.1.a to 5.8.a as a function of the side of a square field. For reasons of comparison, $S_{c}$ was measured with a mini-phantom, also with a water-equivalent build-up cap in two ${ }^{60} \mathrm{Co}$ beams and in a $4 \mathrm{MV}$ x-ray beam. Within the experimental uncertainty of approximately $0.5 \%$, both methods yielded the same results. For higher nominal beam energies, larger differences were observed [73].

It can be noted that similar to the $S_{c p}$ curves the $S_{c}$ curves from the GE-CGR accelerator beams are much steeper than those of other machines. An overall variation of the $S_{c}$ values of the order of $15 \%$ is observed for the GE-CGR accelerators compared with $8 \%$ for the other accelerators when varying the field sizes from $4 \mathrm{~cm} \times 4 \mathrm{~cm}$ up to $40 \mathrm{~cm} \times 40 \mathrm{~cm}$. 
5.

Table 5.1 Some characteristics of the beams of which data are available. Indicated are: nominal accelerator potential $(E)$, quality index $(Q l)$ and whether $S_{c}$ and $S_{p}$ data for square and rectangular fields $(r)$, or only for square fields (s) are available.

\begin{tabular}{|c|c|c|c|c|c|c|}
\hline$\#$ & Manufacturer & Type & $E(M V)$ & QI & $\begin{array}{c}\text { square (s) or } \\
\text { fields } \\
s_{e}\end{array}$ & $\begin{array}{c}\text { rectangular }(r) \\
\text { measured } \\
S_{0}\end{array}$ \\
\hline 1 & AECL & Theratron 780 & ${ }^{60} \mathrm{Co}$ & 0.572 & $s$ & $s^{2}$ \\
\hline 2 & Philips & & ${ }^{60} \mathrm{Co}$ & 0.572 & $\mathrm{~s}$ & $s^{2}$ \\
\hline 3 & Philips & & ${ }^{60} \mathrm{Co}$ & 0.572 & $\mathrm{~s}$ & $s^{2}$ \\
\hline 4 & Varian & Clinac-4 & 4 & 0.616 & s & $s$ \\
\hline 5 & $A B B$ & Dynaray-4 & 4 & 0.614 & $s$ & $\mathrm{~s}$ \\
\hline 6 & Philips & SL75-10 & 6 & 0.650 & $s$ & $\mathrm{~s}$ \\
\hline 7 & Siemens & MV & 6 & 0.675 & $\mathrm{~s}$ & $\mathrm{~s}$ \\
\hline 8 & Philips & SL25 & 6 & 0.678 & $s$ & $\mathrm{~s}$ \\
\hline 9 & Varian & Clinac -6 & 6 & 0.650 & $\mathrm{~s}$ & $\mathrm{~s}$ \\
\hline 10 & GE-CGR & Saturne-41 & 6 & 0.670 & $\mathrm{r}$ & $r$ \\
\hline 11 & Philips & SL15 & 6 & 0.680 & $r$ & $r$ \\
\hline 12 & Philips & SL2O & 6 & 0.679 & $r$ & $r$ \\
\hline 13 & $A B B$ & Dynary-20 & 6 & 0.687 & $r$ & $r$ \\
\hline 14 & Philips & SL75-20 & 8 & 0.714 & s & s \\
\hline 15 & Philips & SL75-14 & 8 & 0.714 & $\mathrm{~s}$ & $\mathrm{~s}$ \\
\hline 16 & GE-CGR & Saturne-41 & 10 & 0.729 & $r$ & $r$ \\
\hline 17 & Siemens & Mevatron 74 & 10 & 0.734 & s & s \\
\hline 18 & Philips & SL15 & 10 & 0.737 & $r$ & $r$ \\
\hline 19 & Philips & SL75-20 & 16 & 0.763 & $\mathrm{~s}$ & $\mathrm{~s}$ \\
\hline 20 & $A B B$ & Dynaray-20 & 16 & 0.772 & $r$ & $r$ \\
\hline 21 & Philips & SL2O & 18 & 0.774 & $r$ & $r$ \\
\hline 22 & GE-CGR & Saturne & 18 & 0.758 & s & s \\
\hline 23 & GE-CGR & Saturne & 23 & 0.783 & $\mathrm{~s}$ & $\mathrm{~s}$ \\
\hline 24 & Philips & SL25 & 25 & 0.783 & $r$ & $r$ \\
\hline 25 & GE-CGR & Sagittaire & 25 & 0.783 & $\mathrm{~s}$ & $\mathrm{~s}$ \\
\hline
\end{tabular}

AECL/Theratronics, Kanata, Canada; GE-CGR, Buc, France; ABB, Baden, Switzerland; Varian, Palo Alto, USA; Philips, Crawley, UK; Siemens, Erlangen, Germany.

$2 \quad S_{p}$ data for the ${ }^{60} \mathrm{Co}$ beams were taken from reference $[8]$ and recalculated to $d_{\text {rer. }}$. 
5.

\section{cobalt- 60 beams}
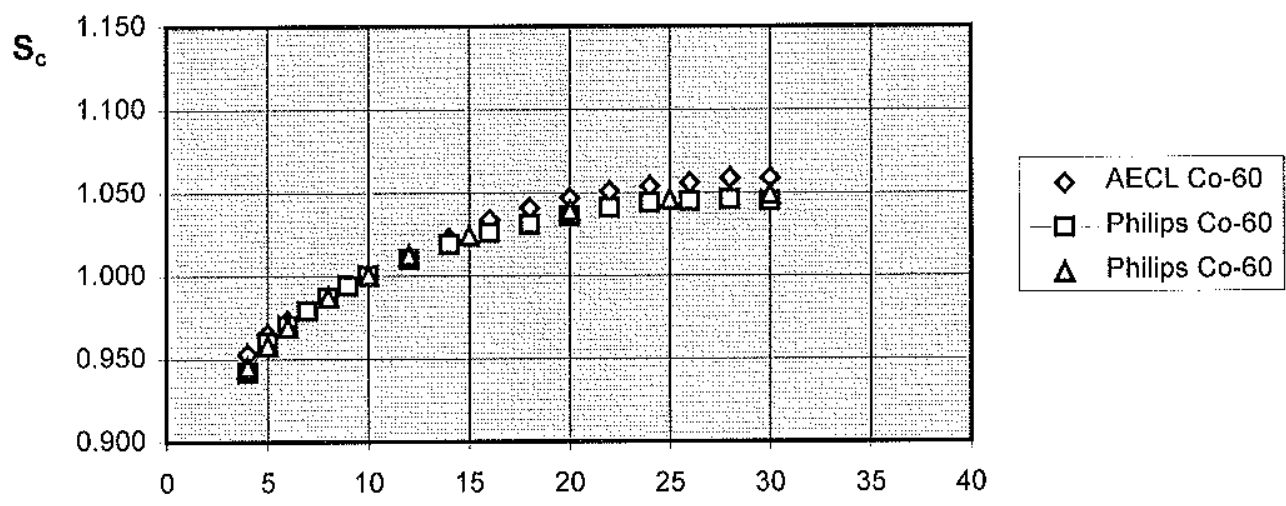

a) side of square field $(\mathrm{cm})$
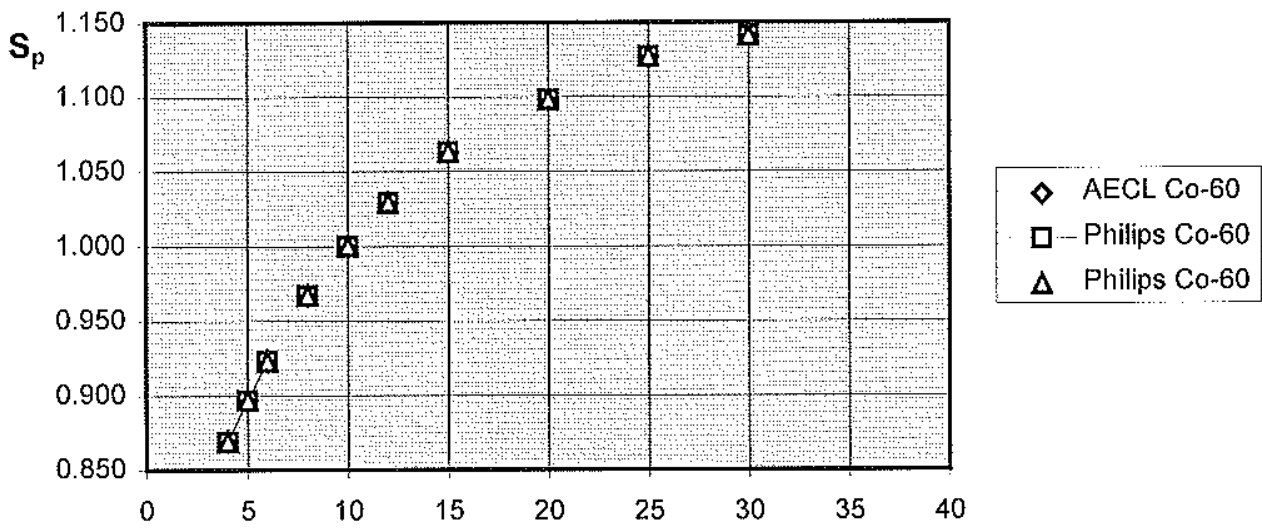

b) side of square field $(\mathrm{cm})$
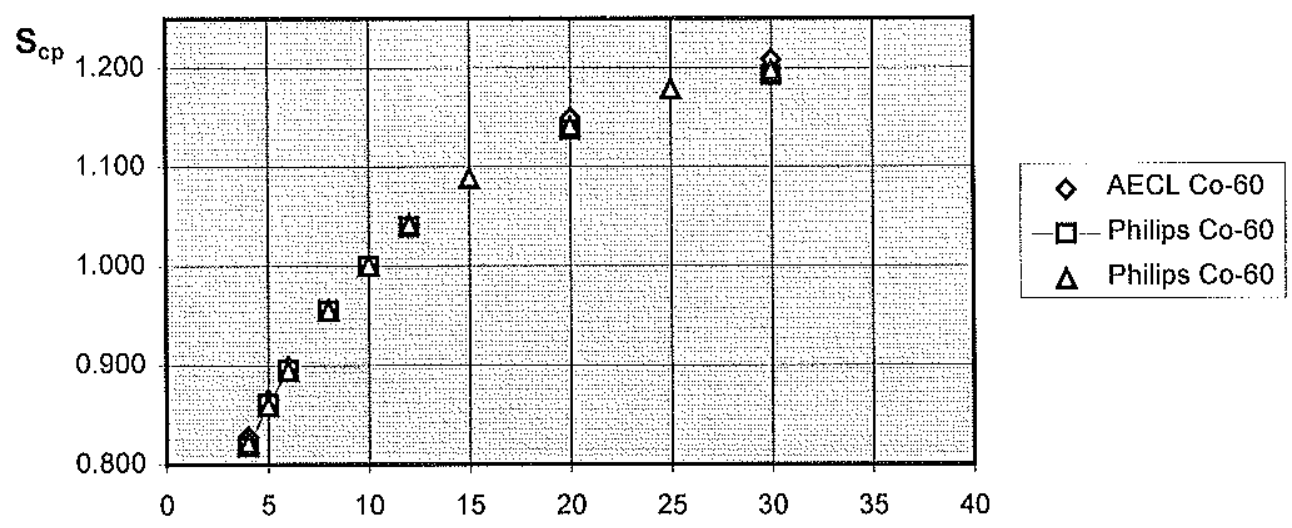

c) side of square field $(\mathrm{cm})$

Figure $5.1 \mathrm{~S}_{\mathrm{c}}, \mathrm{S}_{\mathrm{p}}$ and $\mathrm{S}_{\mathrm{cp}}$ data as a function of the side of a square field of ${ }^{60} \mathrm{Co}$ beams (beams 1 3). Data are normalized at depth $d_{\text {reif }}$. 
5.

\section{MV beams}

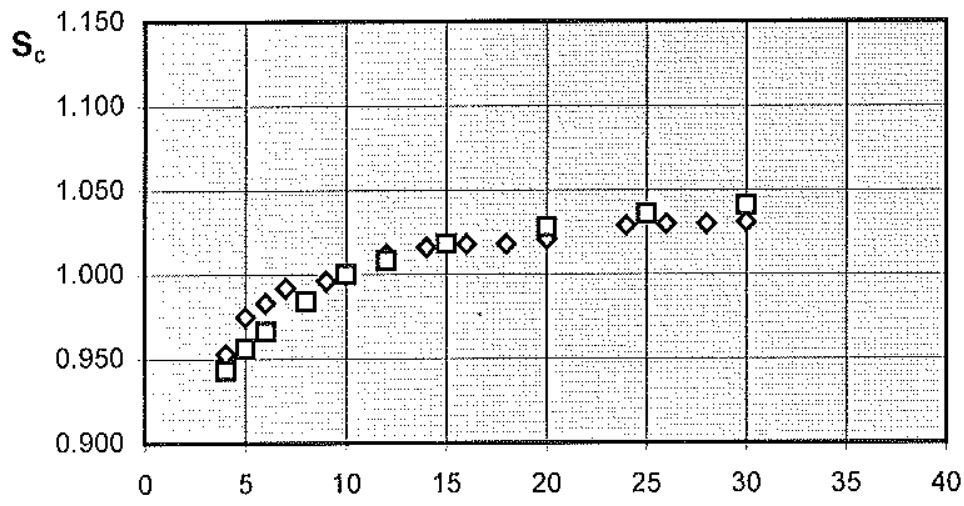

$\diamond$ Clinac4 4MV

a) side of square field $(\mathrm{cm})$

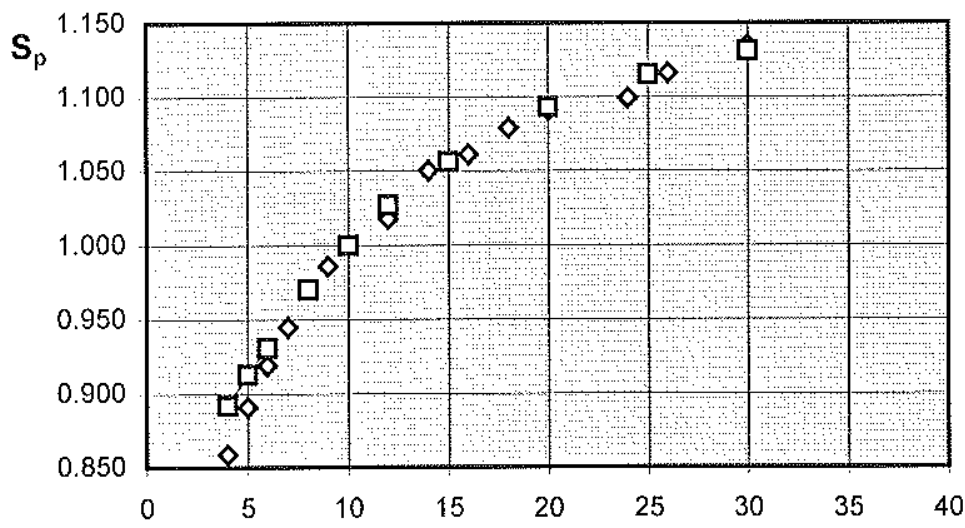

$\diamond$ Clinac4 4MV

ㅁ. ABB4 4MV

b) side of square field $(\mathrm{cm})$
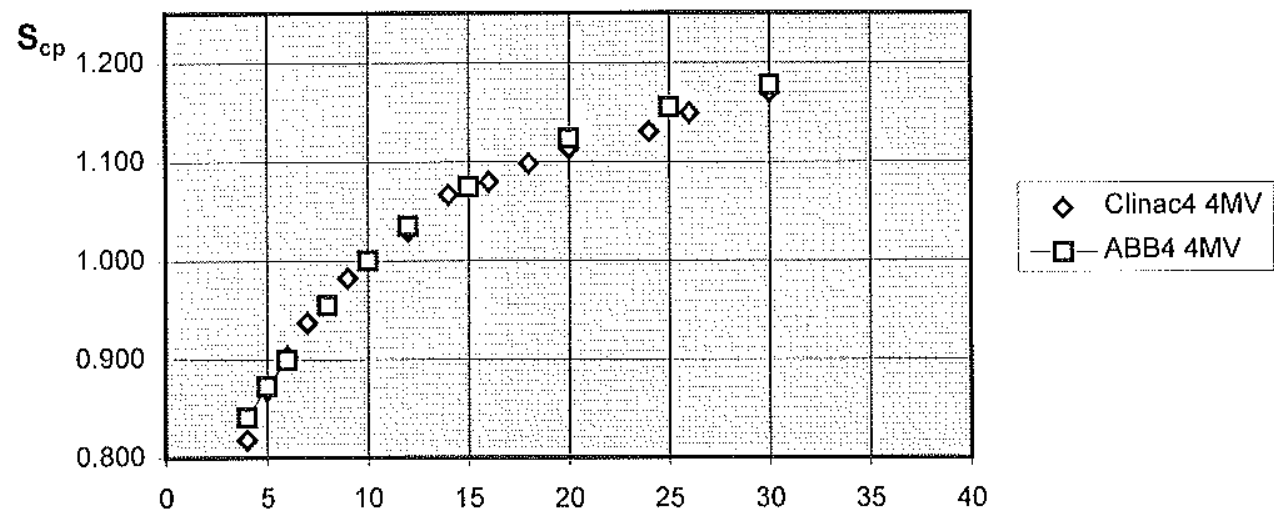

c) side of square field $(\mathrm{cm})$

Figure 5.2 $\mathrm{S}_{\mathrm{c}^{\prime}} \mathrm{S}_{\mathrm{p}}$ and $\mathrm{S}_{\mathrm{cp}}$ data as a function of the side of a square field of $4 \mathrm{MV}$ beams (beams 45). Data are normalized at depth $d_{\text {ret }}$. 
5.

\section{$6 \mathrm{MV}$ beams}

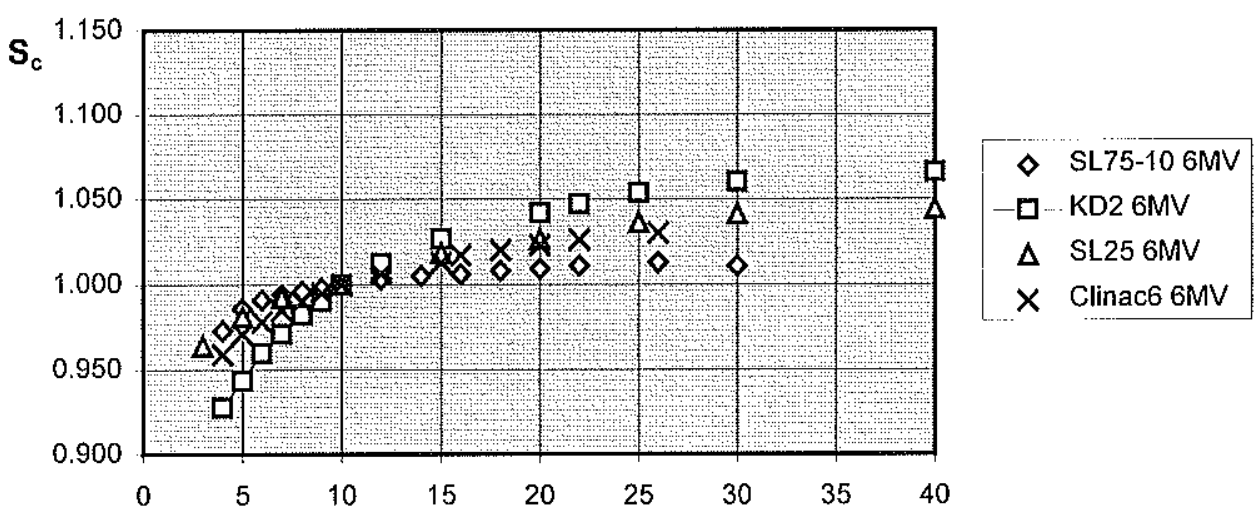

a) side of square field $(\mathrm{cm})$
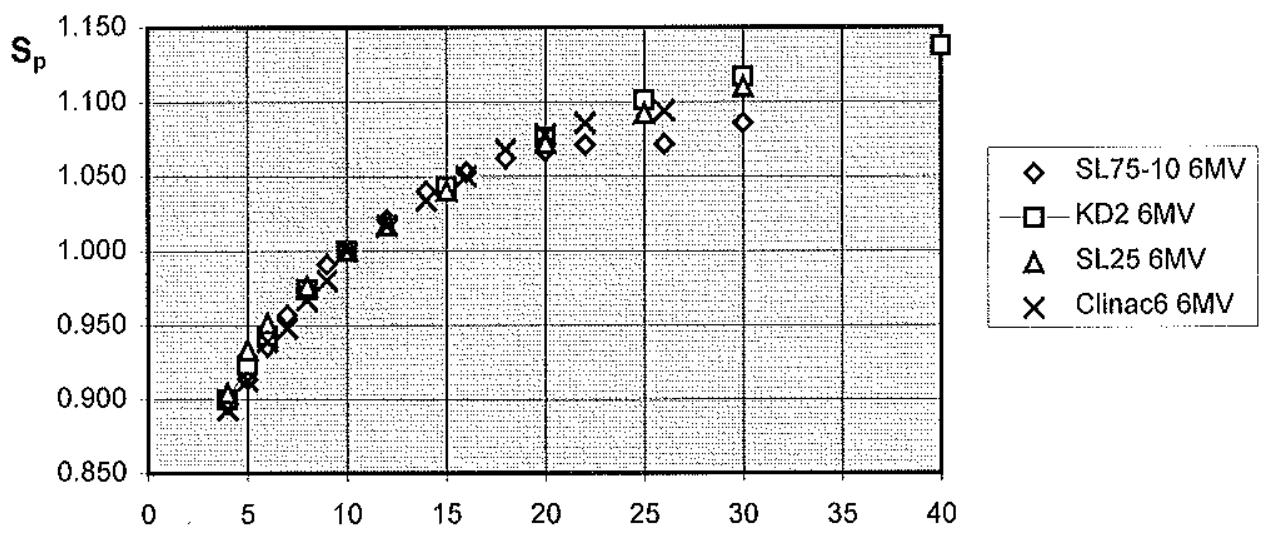

b) side of square field $(\mathrm{cm})$

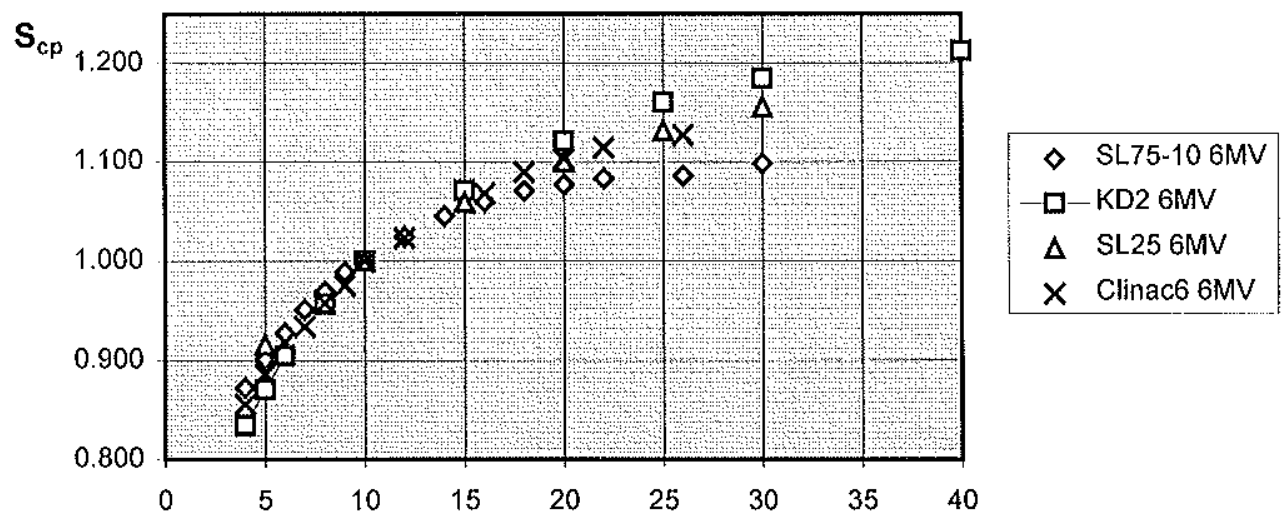

c) side of square field $(\mathrm{cm})$

Figure 5.3 $S_{c}, S_{p}$ and $S_{c p}$ data as a function of the side of a square field of the first four $6 \mathrm{MV}$ beams of table 5.1 (beams 6-9). Data are normalized at depth $d_{r a 1}$. 
5.

6 MV beams (continued)

$\mathbf{S}$

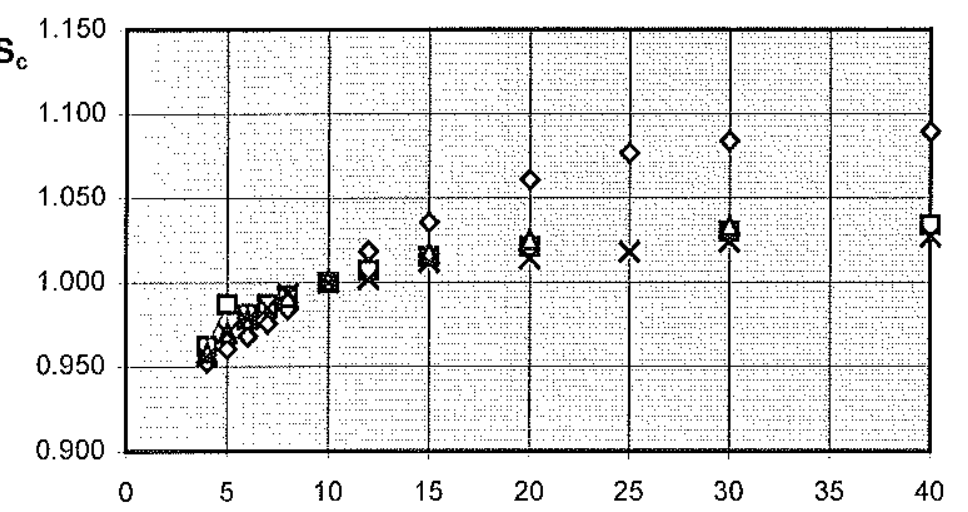

a) side of square field $(\mathrm{cm})$

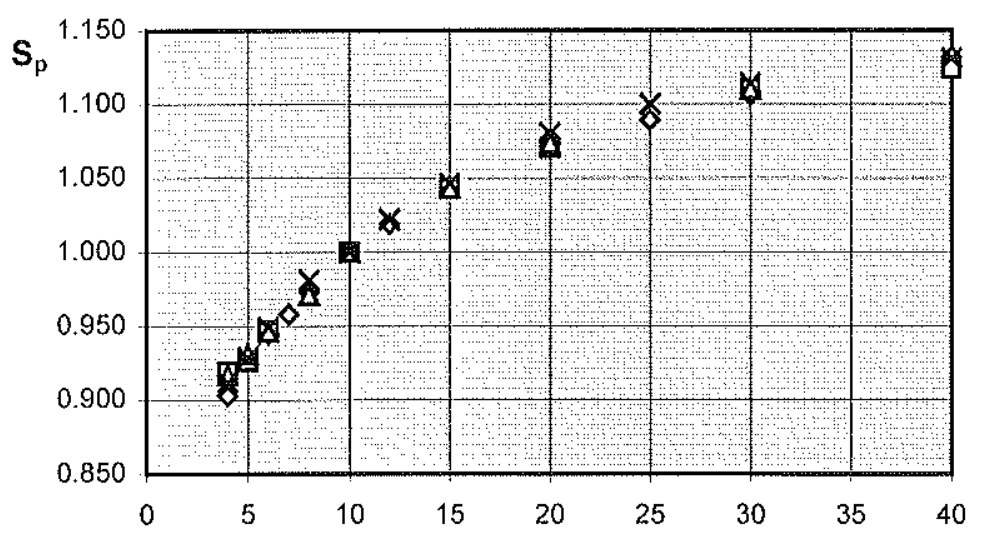

b) side of square field $(\mathrm{cm})$

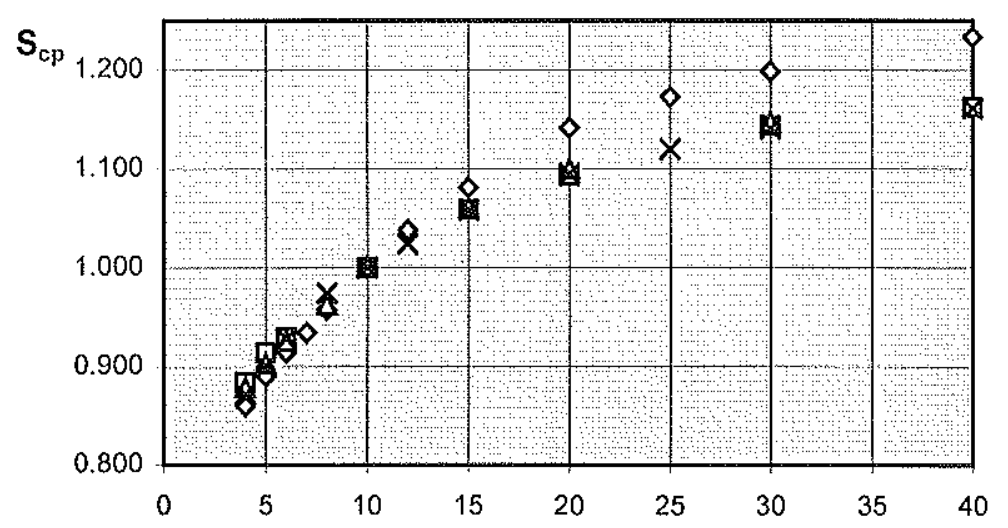

c) side of square field $(\mathrm{cm})$ $\diamond$ SAT41 6MV

- -SL15 6MV

$\triangle$ SL20 6MV

$\times \quad A B B 206 M V$

$$
\begin{gathered}
\diamond \text { SAT41 6MV } \\
\square-S L 156 M V \\
\Delta \quad \text { SL20 6MV } \\
\times \quad \text { ABB20 6MV } \\
\hline
\end{gathered}
$$
$\diamond$ SAT41 6MV
口. SL15 6MV
$\triangle$ SL20 6MV
$\times$ ABB20 $6 \mathrm{MV}$

Figure 5.4 $\mathrm{S}_{c^{\prime}} \mathrm{S}_{\mathrm{p}}$ and $\mathrm{S}_{\mathrm{cp}}$ data as a function of the side of a square field of $6 \mathrm{MV}$ beams (continued with beams 10-13). Data are normalized at depth $\mathrm{d}_{\text {ref }}$. 
5.

\section{MV beams}

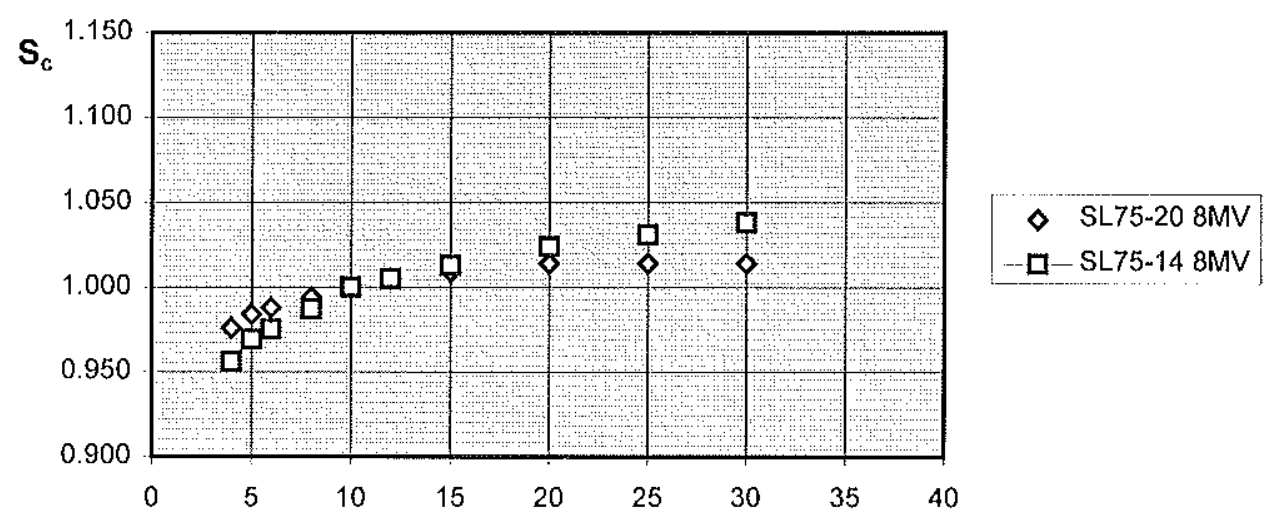

a) side of square field $(\mathrm{cm})$

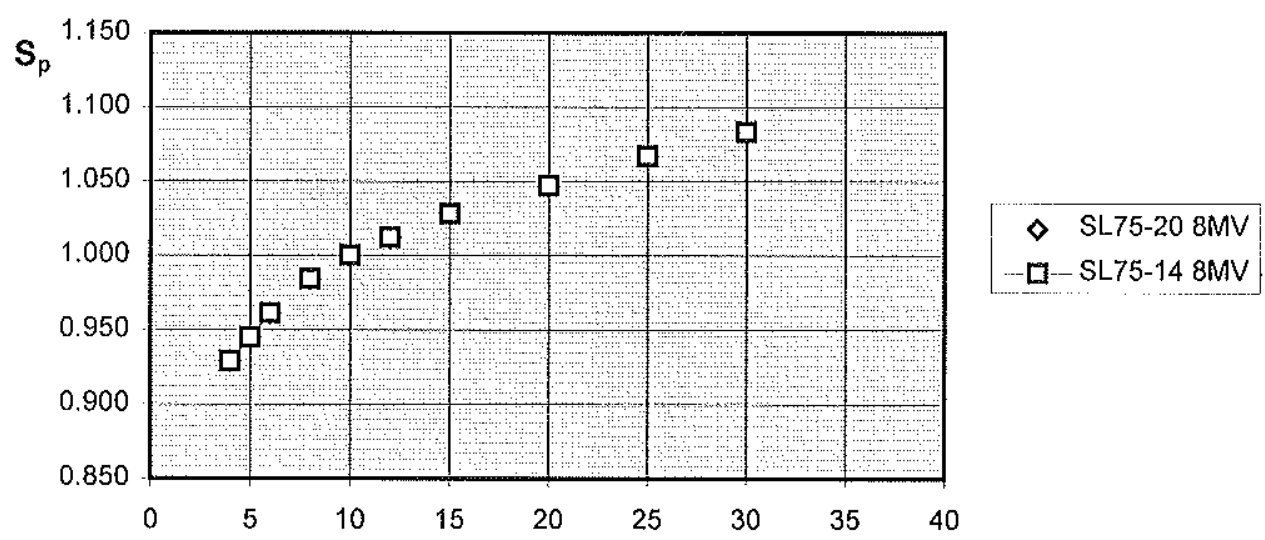

b) side of square field $(\mathrm{cm})$

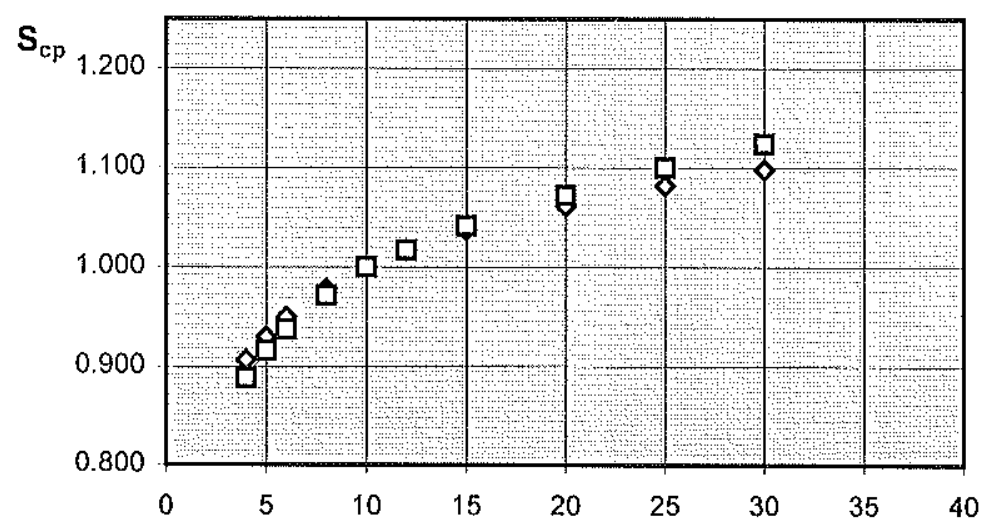

$\diamond$ SL75-20 8MV

ㅁ. SL75-14 8MV

c) side of square field $(\mathrm{cm})$

Figure $5.5 \mathrm{~S}_{c}, \mathrm{~S}_{\mathrm{p}}$ and $\mathrm{S}_{c p}$ data as a function of the side of a square field of $8 \mathrm{MV}$ beams (beams 14-15). Data are normalized at depth $d_{\text {ref }}$. 
5.

\section{MV beams}

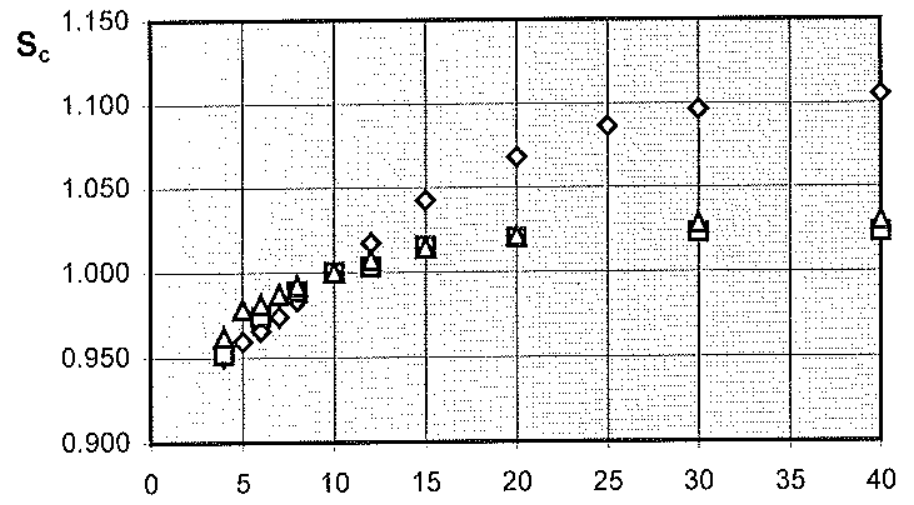

\section{- SAT41 10MV}

口--Mevatron74 10MV

$\triangle$ SL15 10MV

a) side of square field $(\mathrm{cm})$

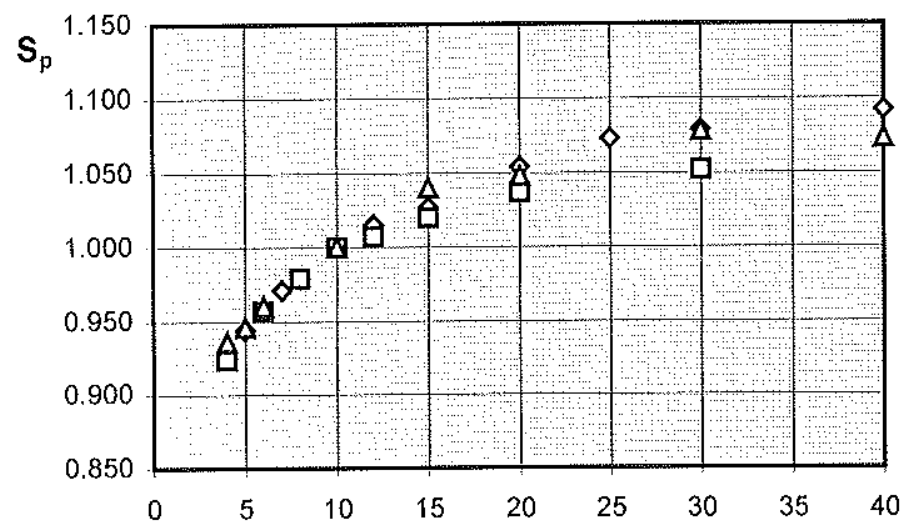

$\diamond$ SAT41 10MV

- $\square$. Mevatron74 10MV

$\triangle$ SL15 10MV

b) side of square field $(\mathrm{cm})$

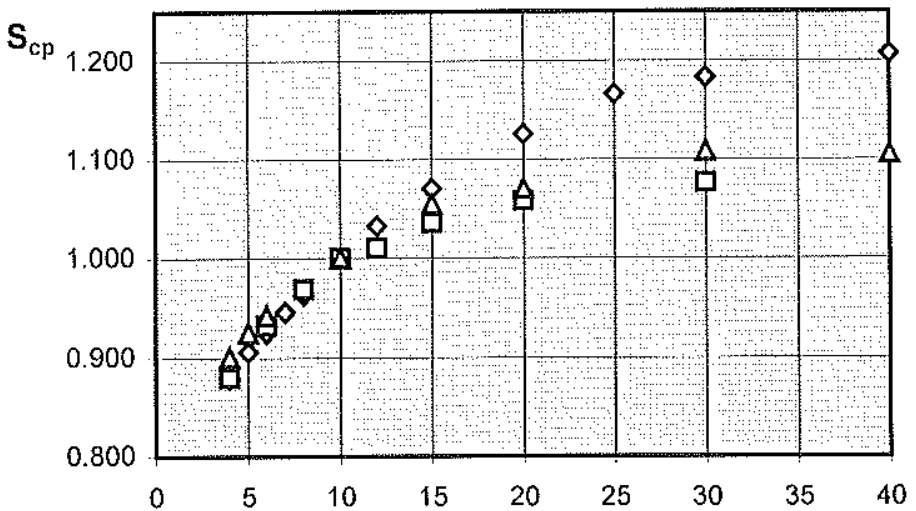

$\diamond$ SAT41 10MV

- - Mevatron74 10MV

A SL15 10MV

c) side of square field $(\mathrm{cm})$

Figure 5.6 $S_{c}, S_{p}$ and $S_{c p}$ data as a function of the side of a square field of $10 \mathrm{MV}$ beams (beams 16-18). Data are normalized at depth $d_{\text {ref }}$. 
5.

16-18 MV

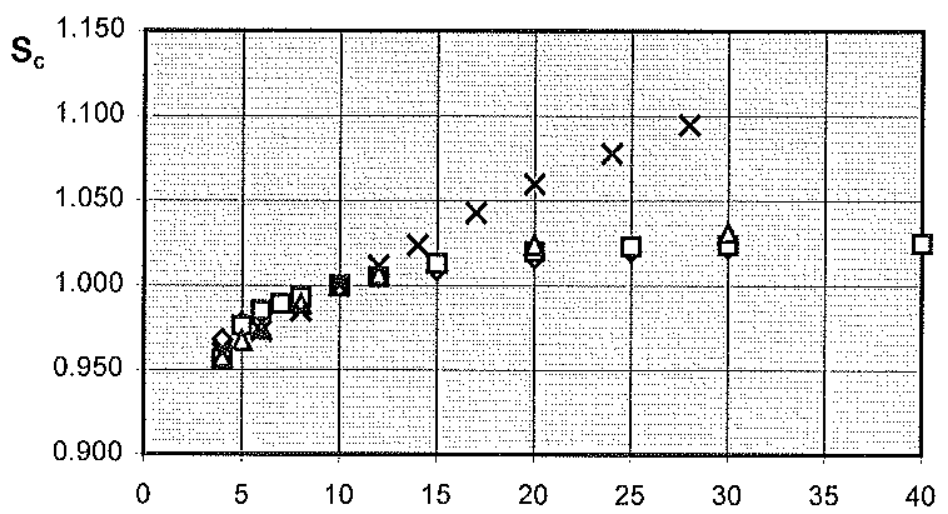

a) side of square field $(\mathrm{cm})$

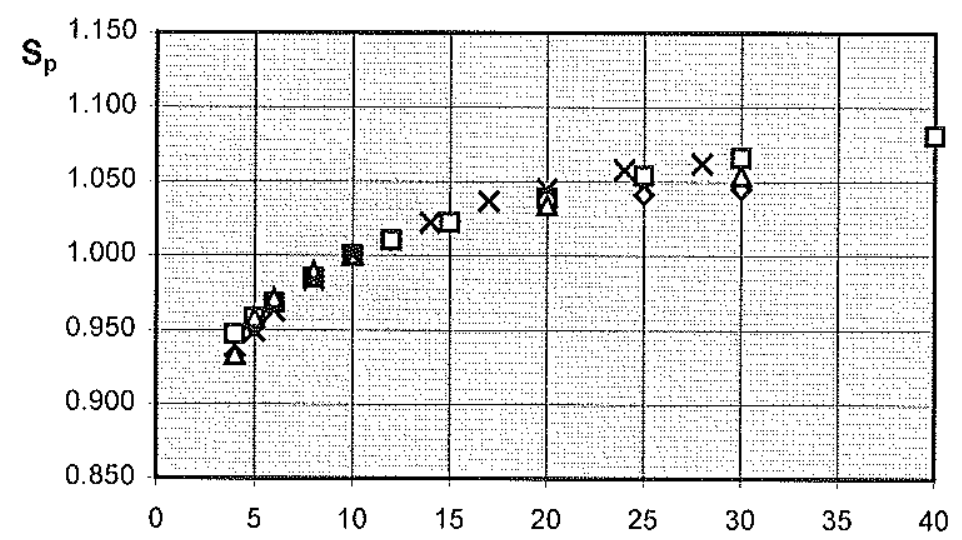

b) side of square field $(\mathrm{cm})$

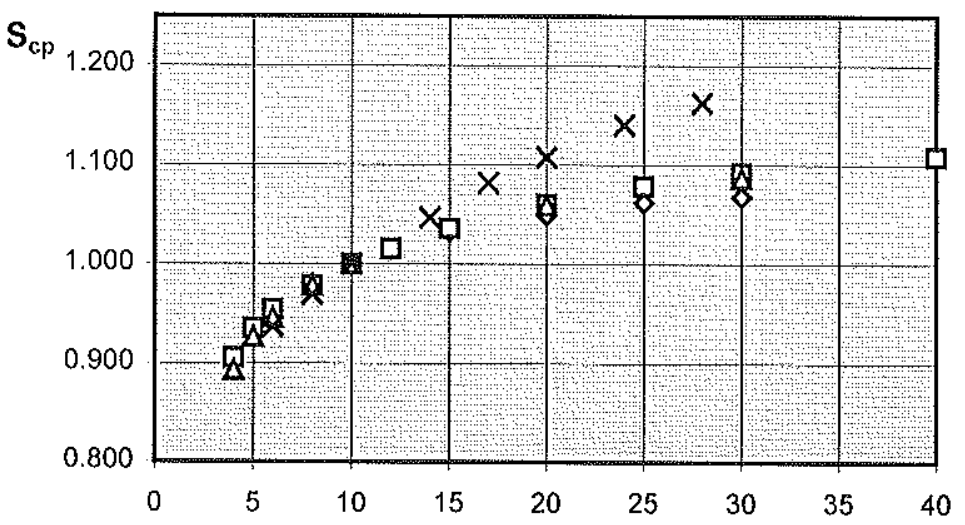

$\diamond$ SL75-20 16MV

$-\square-$ ABB20 $16 \mathrm{MV}$

$\triangle$ SL20 18MV

$\times$ Saturne 18MV

Figure $5.7 S_{c}, S_{p}$ and $S_{c p}$ data as a function of the side of a square field of 16-18 MV beams (beams 19-22). Data are normalized at depth $d_{\text {ref }}$. 
5.

\section{3-25 MV}
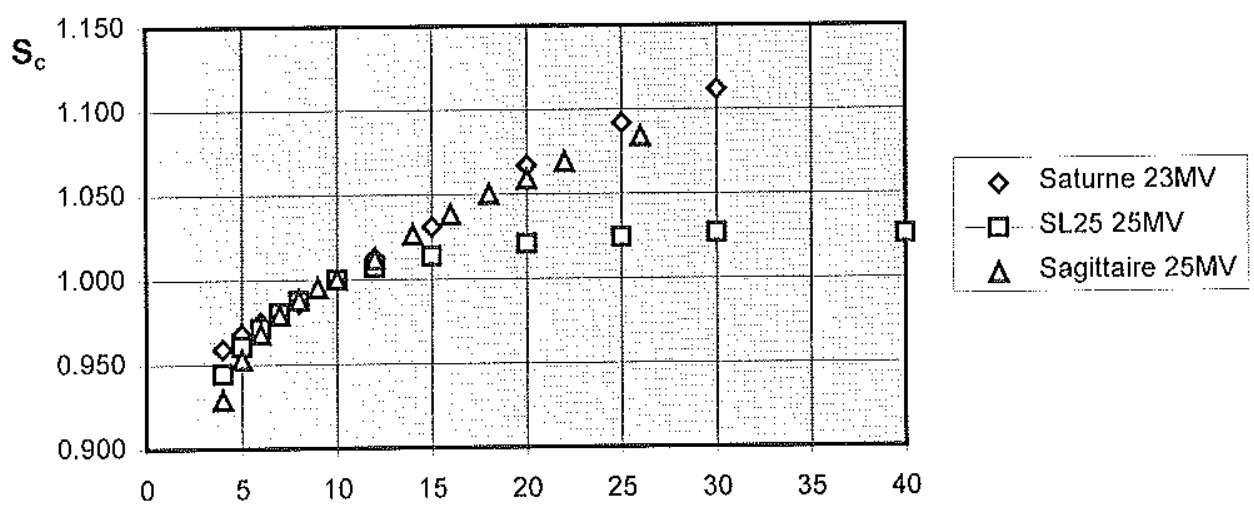

a) side of square field $(\mathrm{cm})$

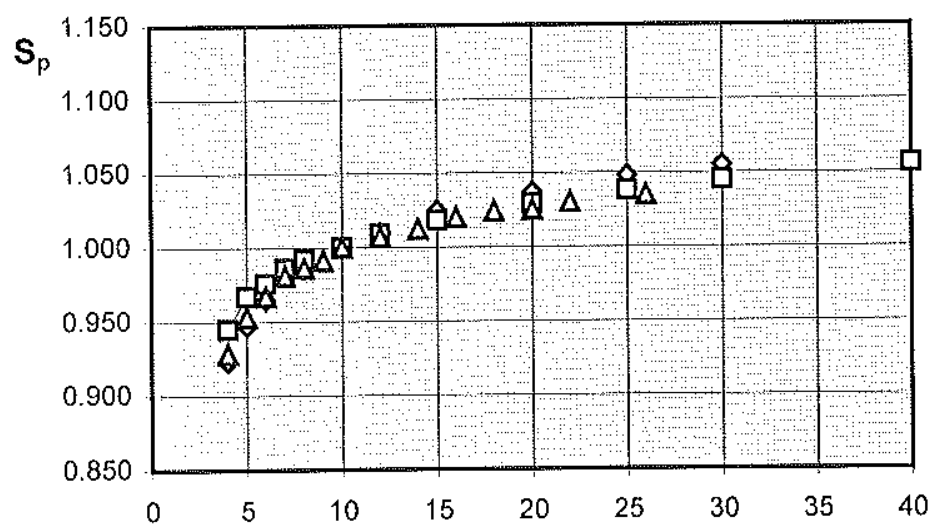

$\diamond$ Saturne $23 \mathrm{MV}$

- $\mathrm{SL25} 25 \mathrm{MV}$

$\triangle$ Sagittaire $25 \mathrm{MV}$

b) side of square field $(\mathrm{cm})$

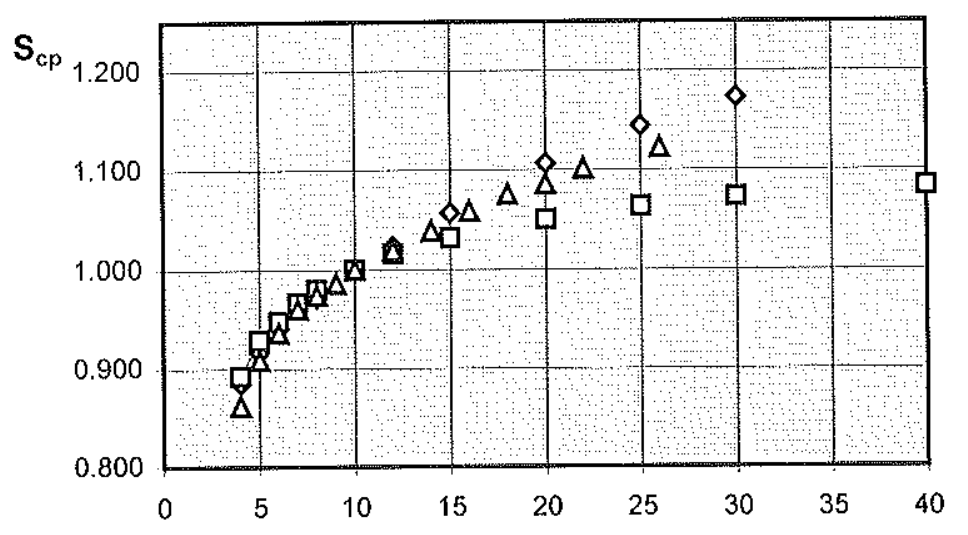

$\diamond$ Saturne 23MV

口. SL25 25MV

$\Delta$ Sagittaire $25 \mathrm{MV}$

c) side of square field $(\mathrm{cm})$

Figure $5.8 \mathrm{~S}_{c}, \mathrm{~S}_{\mathrm{p}}$ and $\mathrm{S}_{\mathrm{cp}}$ data as a function of the side of a square field of 23-25 MV beams (beams 23-25). Data are normalized at depth $d_{\text {ret }}$. 
5.

\subsection{2 $\quad S_{c}$ for rectangular fields}

For several beams, $S_{c}$ data have been determined for rectangular fields as a function of the independent setting of the $X$ and $Y$ collimators (see table 5.1). These experimental data showed that $S_{c}$ is an asymmetrical function of the $X$ and $Y$ collimator settings, as observed by various other groups $[24,33,57,58]$. The order of magnitude of this effect (the collimator exchange effect, CEE) is about $2 \%$ for the linear accelerators investigated in this report. Figures 5.9.a and 5.9.b illustrate this effect for two different machines.

\section{SL15, 10 MV}

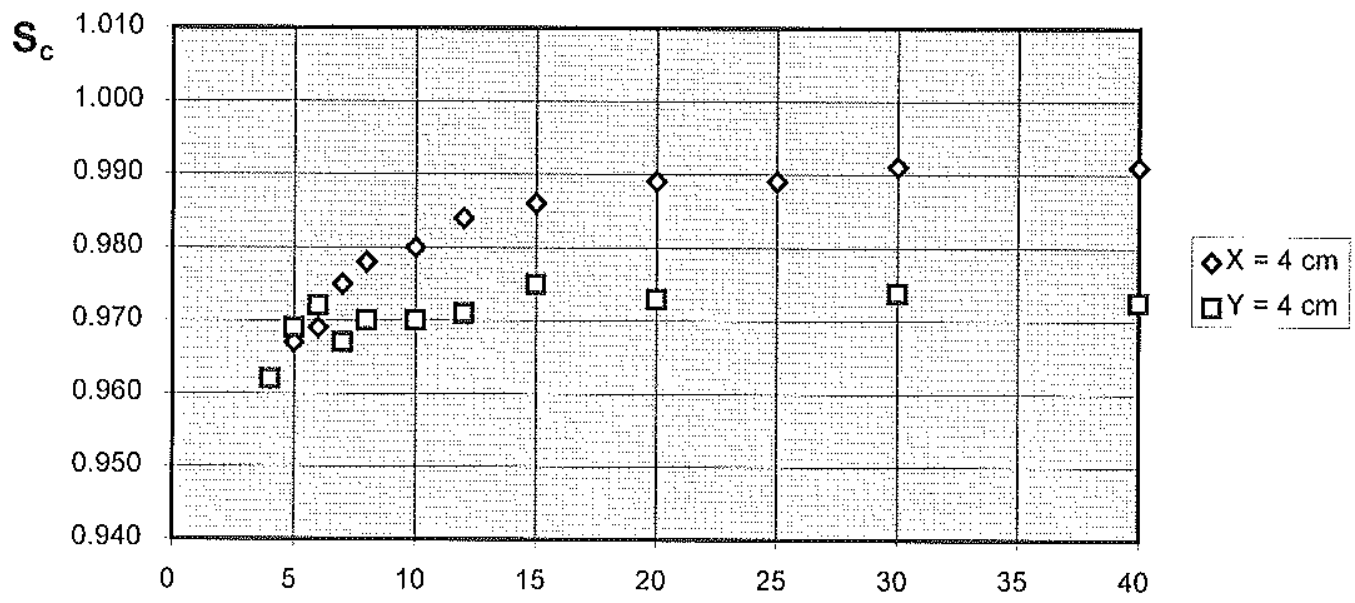

a) long field side $(\mathrm{cm})$

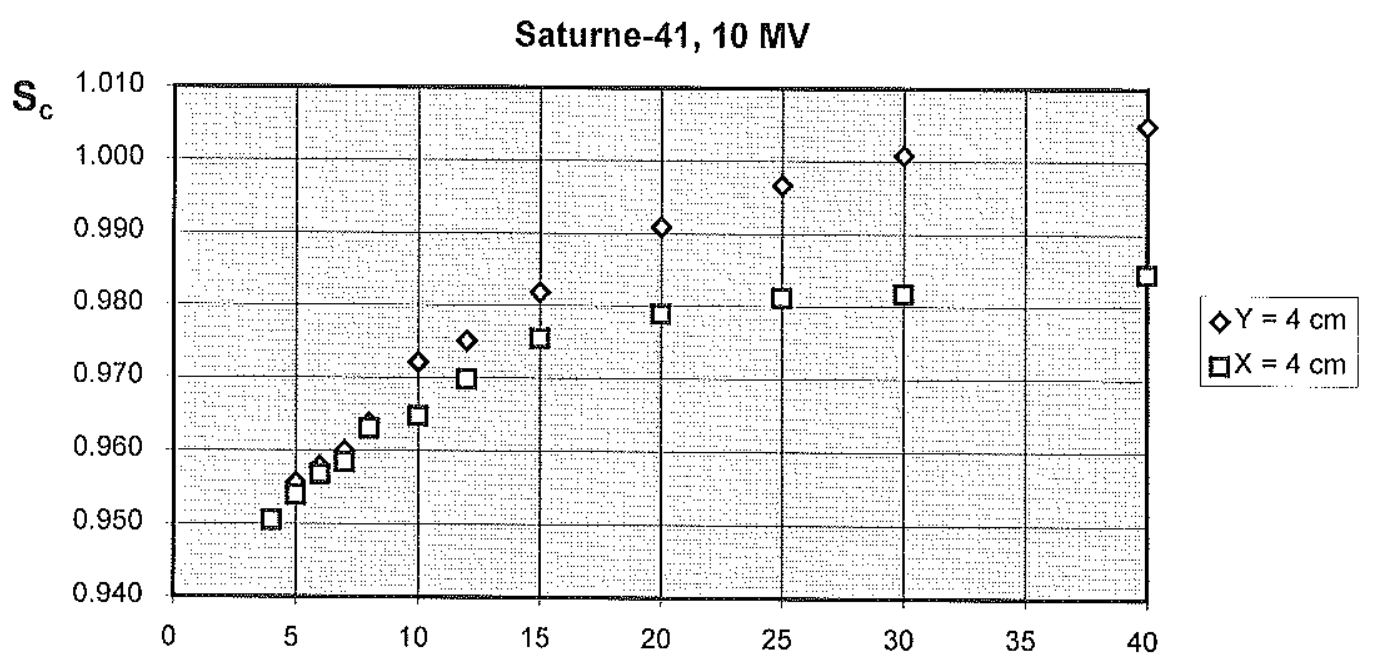

b) long field side $(\mathrm{cm})$

Figure 5.9 The collimator exchange effect measured in a Philips SL15 $10 \mathrm{MV}$-ray beam (a) and in a GE-CGR Saturne-41 $10 \mathrm{MV} x$-ray beam (b). $S_{c}$ is shown as a function of the long field side, setup with either the $X$ or $Y$ collimator fixed to $4 \mathrm{~cm}$. 


\subsubsection{Influence of SSD and $d_{\text {ref }}$ on $S_{c}$}

The influence of the choice of SSD and $d_{r e f}$ on the value of $S_{c}$ was measured in a high and a low quality photon beam. Data were obtained from a $25 \mathrm{MV} \times$-ray beam for $S S D=90 \mathrm{~cm}$ and $d_{\text {ref }}=20 \mathrm{~cm}$, for $S S D=100 \mathrm{~cm}$ and $d_{\text {ref }}=10 \mathrm{~cm}$, and for $S S D=150 \mathrm{~cm}$ and $d_{r e f}=10 \mathrm{~cm}$. These data are presented as a function of the the side of a square field at SAD in figure 5.10.a. A similar experiment was performed in a $4 \mathrm{MV}$ beam, where the following combinations of SSD and $d_{\text {ref }}$ were used (in $\mathrm{cm}$ ): $80 / 10 ; 105 / 10 ; 65 / 10$ and $80 / 15$. The results are shown in figure $5.10 . \mathrm{b}$.
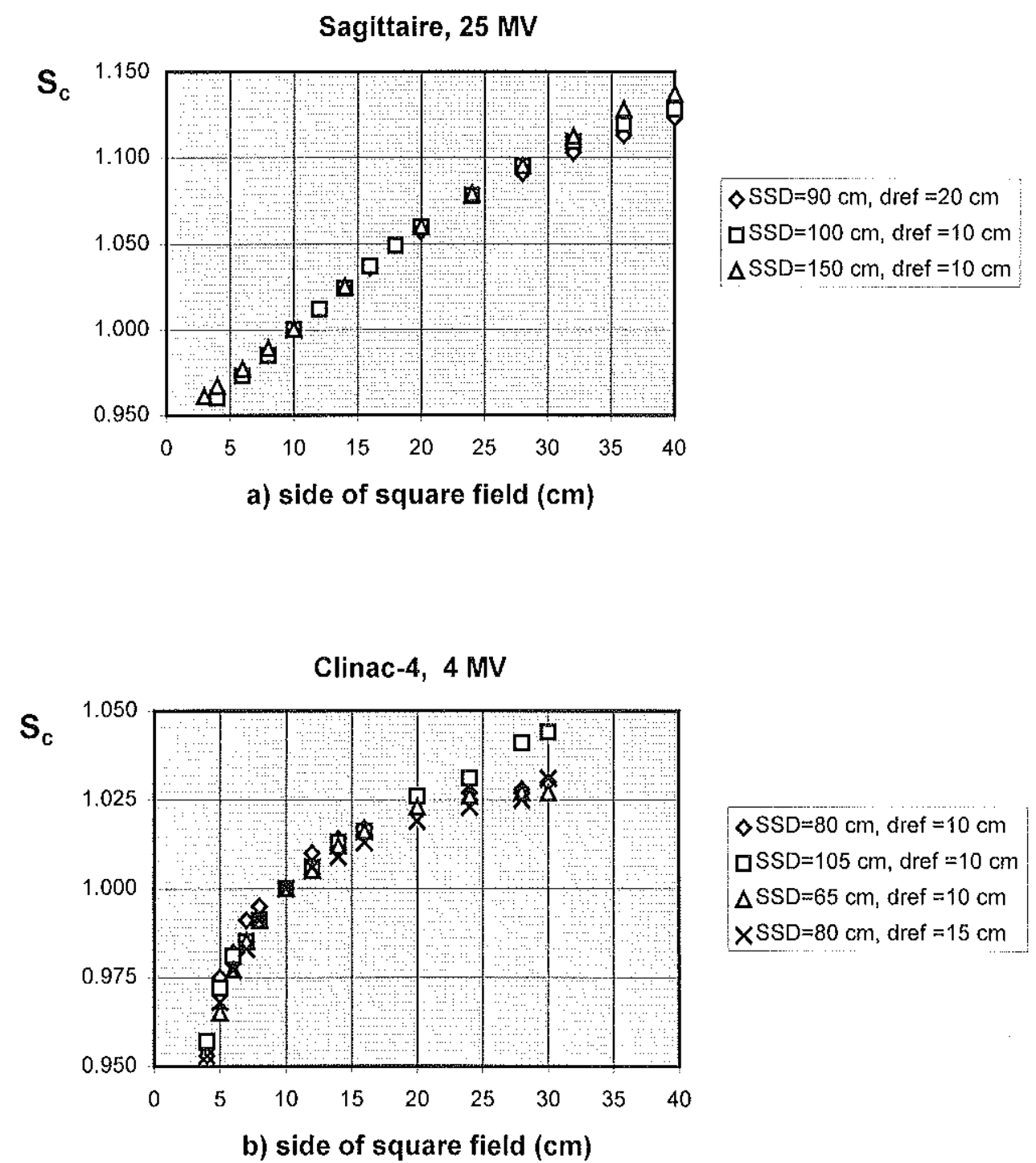

Figure 5.10 The influence of the source-surface distance on $S_{c}$ measured in two different beams, $25 \mathrm{MV}$ (a) and $4 \mathrm{MV}$ (b); see text for further details. 
5.

From these results it can be concluded that:

- $S_{c}$ is independent of the choice of SSD within the range of clinically applied distances;

- $S_{c}$ does not depend on the choice of the depth $d_{r e f}$ provided $d_{r e f}$ is beyond the range of the contaminating electrons.

\subsubsection{Influence of a wedge on $\mathrm{S}_{\mathrm{c}}$}

The influence of the presence of a wedge on the value of $S_{c}$ has been the subject of several studies $[22,61,75]$. As an example, $S_{c}$ data of the 6,10 and 25 MV photon beams of a Saturne-43 linear accelerator (GE-CGR, Buc, France) are shown here. These data are presented in figure 5.11 as a function of the side of the square field, obtained with a mini-phantom at $S S D=100 \mathrm{~cm}$ and a measurement depth of $10 \mathrm{~cm}$. The $10 \mathrm{~cm} \times 10 \mathrm{~cm}$ field is used as the reference field. Open symbols are used in this graph for the open beams, closed symbols for the wedged beams. For a specified field size, the relative wedge transmission factor, RWF, can be deduced from the ratio of $S_{c, w}$ and $S_{c, o p e n}$. For this machine, typical values of the RWF are found of approximately $2.5 \%$ for the largest square field size. For other machines, however, the values of RWF are more or less pronounced, depending on the construction and material of the wedge and its position in the head of the treatment machine. Values for RWF up to $8 \%$ have been reported $[22,61,75]$.

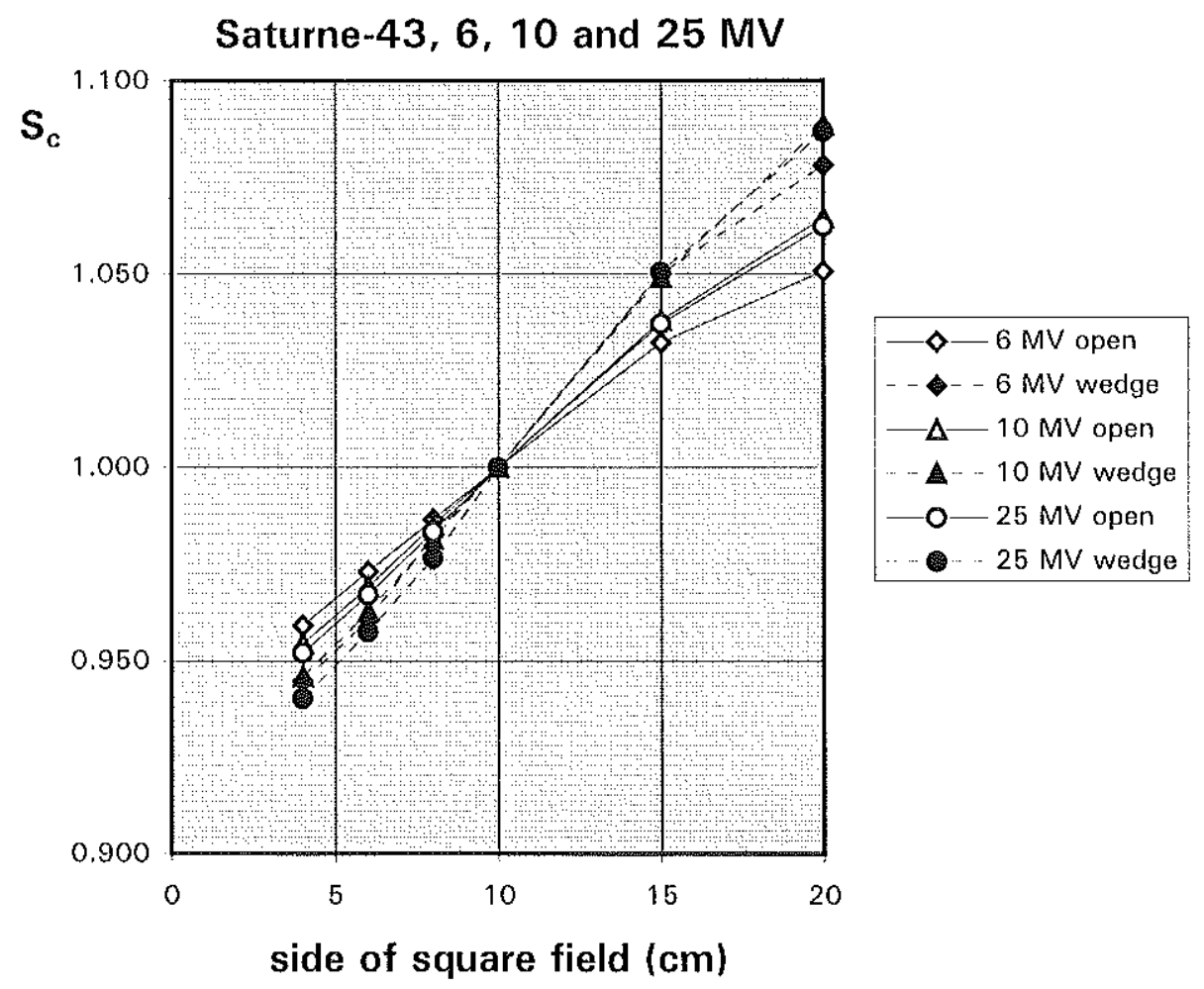

Figure $5.11 \mathrm{~S}_{\mathrm{c}}$ data with and without a $60^{\circ}$ wedge in the beam as a function of the side of a square field for three different nominal photon beam energies of a Saturne- 43 linear accelerator. 


\subsection{Phantom scatter correction factors, $S_{p}$}

\subsection{1 $\quad S_{p}$ for square fields}

$S_{p}$ data have been derived from the $S_{c p}$ and $S_{c}$ data for each photon beam quality under investigation and for each field size according to expression (2.1.3). The results are presented as a function of the side of a square field in figures 5.1.b to 5.8.b. Within the experimental uncertainty, which is less than approximately $1 \%$, the $S_{p}$ curves of different machines with the same $\mathrm{Ql}$ coincide. The overall variation in $S_{p}$ with field size depends strongly on the beam quality. For $S_{p}$ defined at the reference depth of $10 \mathrm{~cm}$, this variation is 0.87 to 1.15 for a ${ }^{60} \mathrm{Co}$ beam, and 0.93 to 1.06 for a $25 \mathrm{MV}$ x-ray beam for the range of field sizes of 4 $\mathrm{cm} \times 4 \mathrm{~cm}$ up to $40 \mathrm{~cm} \times 40 \mathrm{~cm}$.

The $S_{p}$ data for several square field sizes are shown as a function of the quality index in figure 5.12 . From this plot it can be concluded that $S_{p}$ is a smooth function of the beam quality.

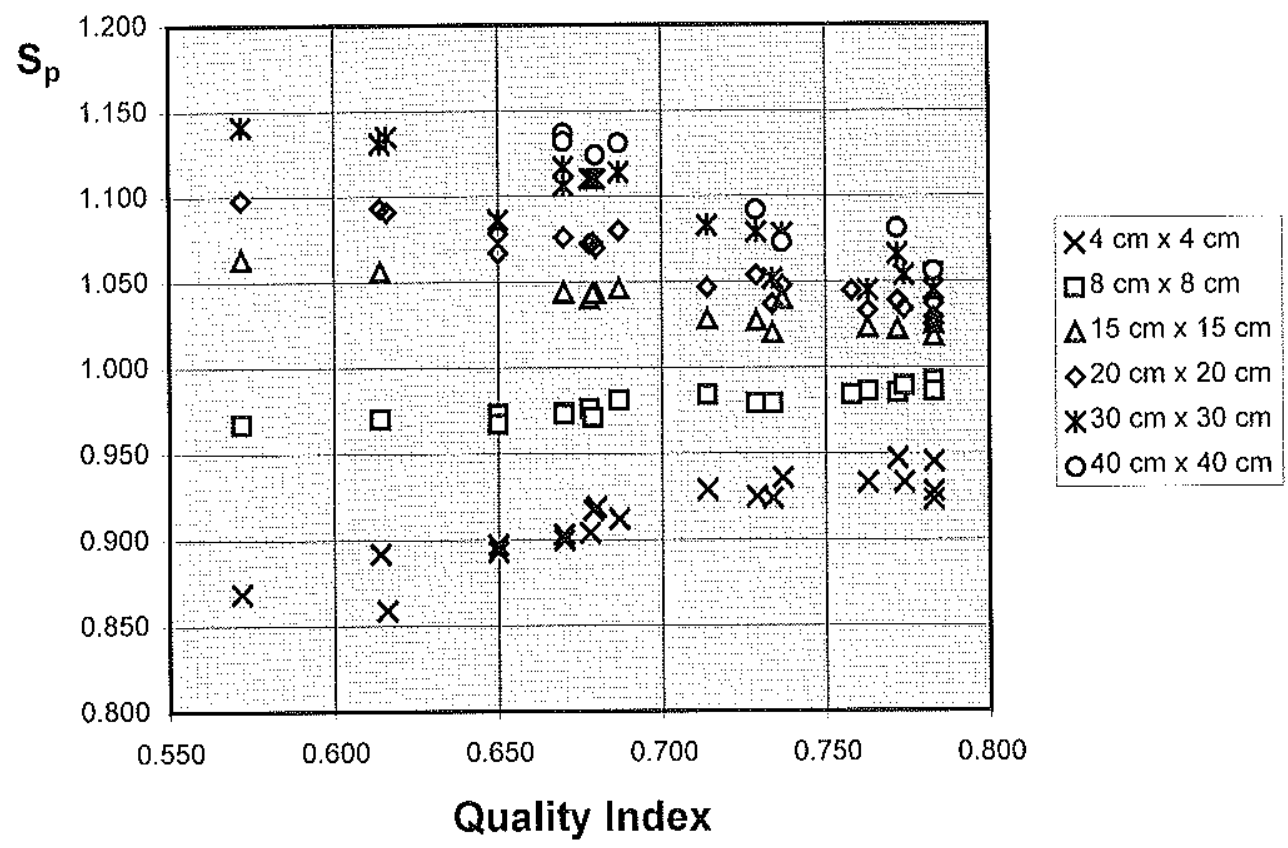

Figure $5.12 \mathrm{~S}_{\mathrm{p}}$ data for a number of square field sizes, presented as a function of the quality index. 
Based on the same experimental data, a complete set of phantom scatter factors was constructed by Storchi and van Gasteren [56] as a function of the field size and the quality index (table 5.2). The authors state that the error, made by applying the phantom scatter factor values from the table rather than measuring them separately, is less than $1 \%$.

Table 5.2 The phantom scatter factor $S_{p}$ defined at the reference depth of $10 \mathrm{~cm}$. This table has been computed from $S_{p}$ values measured at 27 different beam qualities for square fields of various sizes. (From [56]).

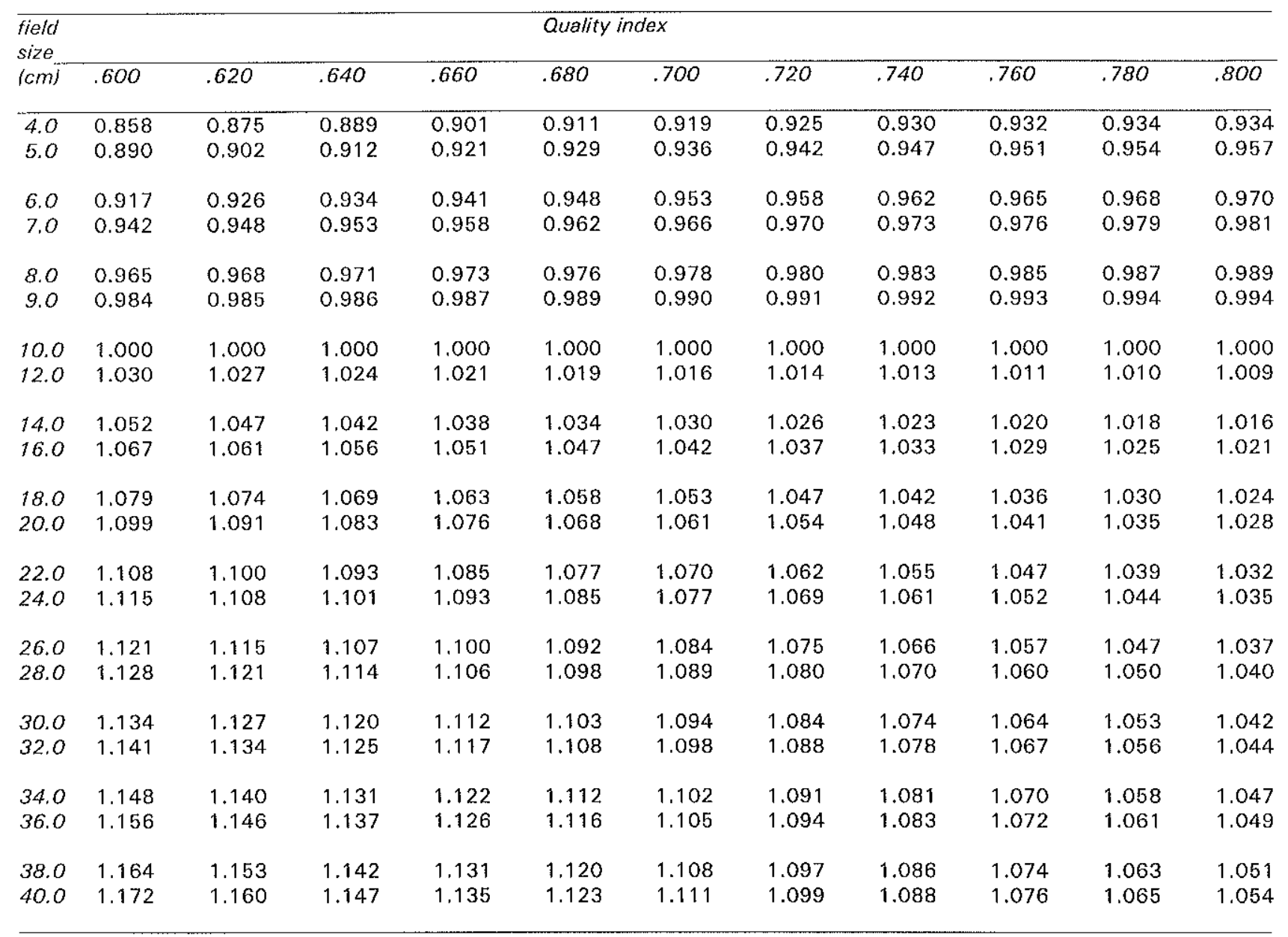

These data have been used to calculate phantom scatter correction factors for use in the isocentric formalism, $S_{p}$ iso. Results are presented in table 5.3. The relations between the quantities in the fixed SSD and in the isocentric formalism are given in Appendix 8.3 of this report. For the conversion of $S_{p}$ data in $S_{p}$ iso data, expression (8.3.7) was used. Differences between the tables amount up to $0.9 \%$, especially for the low energy beams and the larger field sizes [71]. 
5.

Table 5.3 The phantom scatter factor $S_{p}$ iso, defined at the reference depth of $10 \mathrm{~cm}$ for the isocentric treatment set-up. This table has been derived from the data of table 5.2.

\begin{tabular}{|c|c|c|c|c|c|c|c|c|c|c|c|}
\hline \multicolumn{5}{|l|}{$\begin{array}{l}\text { field } \\
\text { size }\end{array}$} & \multicolumn{7}{|c|}{ Quality index } \\
\hline$(\mathrm{cm})$ & .600 & .620 & .640 & .660 & .680 & .700 & .720 & .740 & .760 & .780 & .800 \\
\hline 4.0 & 0.859 & 0.877 & 0.892 & 0.904 & 0.914 & 0.921 & 0.926 & 0.931 & 0.931 & 0.932 & 0.931 \\
\hline 5.0 & 0.888 & 0.902 & 0.913 & 0.923 & 0.930 & 0.937 & 0.942 & 0.946 & 0.948 & 0.950 & 0.952 \\
\hline 6.0 & 0.916 & 0.926 & 0.934 & 0.941 & 0.947 & 0.952 & 0.957 & 0.961 & 0.963 & 0.966 & 0.968 \\
\hline 7.0 & 0.940 & 0.947 & 0.953 & 0.959 & 0.963 & 0.967 & 0.970 & 0.973 & 0.975 & 0.977 & 0.979 \\
\hline 8.0 & 0.962 & 0.967 & 0.970 & 0.974 & 0.976 & 0.978 & 0.981 & 0.983 & 0.985 & 0.987 & 0.989 \\
\hline 9.0 & 0.983 & 0.985 & 0.986 & 0.987 & 0.988 & 0.989 & 0.990 & 0.992 & 0.993 & 0.994 & 0.995 \\
\hline 10.0 & 1.000 & 1.000 & 1.000 & 1.000 & 1.000 & 1.000 & 1.000 & 1.000 & 1.000 & 1.000 & 1.000 \\
\hline 12.0 & 1.029 & 1.026 & 1.024 & 1.022 & 1.019 & 1.017 & 1.015 & 1.013 & 1.011 & 1.010 & 1.010 \\
\hline 14.0 & 1.053 & 1.049 & 1.044 & 1.039 & 1.035 & 1.030 & 1.027 & 1.024 & 1.021 & 1.018 & 1.017 \\
\hline 16.0 & 1.072 & 1.065 & 1.059 & 1.054 & 1.048 & 1.043 & 1.037 & 1.033 & 1.029 & 1.026 & 1.023 \\
\hline 18.0 & 1.085 & 1.078 & 1.072 & 1.066 & 1.060 & 1.054 & 1.047 & 1.042 & 1.037 & 1.032 & 1.027 \\
\hline 20.0 & 1.097 & 1.090 & 1.084 & 1.077 & 1.070 & 1.063 & 1.056 & 1.050 & 1.043 & 1.036 & 1.030 \\
\hline 22.0 & 1.115 & 1.106 & 1.097 & 1.089 & 1.079 & 1.071 & 1.063 & 1.056 & 1.048 & 1.041 & 1.034 \\
\hline 24.0 & 1.124 & 1.114 & 1.106 & 1.097 & 1.087 & 1.079 & 1.070 & 1.062 & 1.053 & 1.044 & 1.037 \\
\hline 26.0 & 1.130 & 1.122 & 1.114 & 1.105 & 1.094 & 1.086 & 1.077 & 1.068 & 1.058 & 1.049 & 1.040 \\
\hline 28.0 & 1.136 & 1.128 & 1.120 & 1.111 & 1.101 & 1.092 & 1.082 & 1.072 & 1.062 & 1.052 & 1.042 \\
\hline 30.0 & 1.142 & 1.134 & 1.126 & 1.117 & 1.107 & 1.097 & 1.087 & 1.076 & 1.066 & 1.055 & 1.045 \\
\hline 32.0 & 1.148 & 1.140 & 1.132 & 1.123 & 1.112 & 1.102 & 1.091 & 1.080 & 1.069 & 1.057 & 1.047 \\
\hline 34.0 & 1.154 & 1.146 & 1.137 & 1.128 & 1.116 & 1.106 & 1.095 & 1.084 & 1.072 & 1.060 & 1.049 \\
\hline 36.0 & 1.160 & 1.152 & 1.142 & 1.132 & 1.121 & 1.110 & 1.098 & 1.087 & 1.075 & 1.063 & 1.051 \\
\hline 38.0 & 1.167 & 1.157 & 1.147 & 1.137 & 1.124 & 1.113 & 1.101 & 1.089 & 1.077 & 1.065 & 1.053 \\
\hline 40.0 & 1.175 & 1.163 & 1.153 & 1.140 & 1.128 & 1.116 & 1.104 & 1.091 & 1.079 & 1.067 & 1.055 \\
\hline
\end{tabular}

\subsection{2 $\quad S_{p}$ for rectangular fields}

Because $S_{p}$ represents the dose due to radiation scattered from the phantom volume to the measuring point at the central axis, $S_{p}$ should be a symmetrical function of the field dimensions (section 3). Consequently, $S_{p}(X=a, Y=b)$ should be equal to $S_{p}(X=b, Y=a)$. For the beams of table 5.1, where $S_{c p}$ and $S_{c}$ were measured at independent settings of the $X$ and $Y$ collimator, this has been confirmed within the experimental uncertainty. 


\subsubsection{Comparison of measured and calculated $S_{p}$ values for rectangular fields}

The method to calculate $S_{p}$ values for rectangular and arbitrarily shaped fields, as described in Appendix 8.4, using $S_{p}$ data measured in square fields, has been applied for a number of beam qualities: $S_{p}$ data were calculated for ${ }^{60} \mathrm{Co}$ and 6 , 10 and 25 MV photon beams for rectangular field sizes with dimensions of $X$ and $Y$ between 4 and $40 \mathrm{~cm}$. The overall agreement between calculation and measurement was better than $0.5 \%[56,70]$.

The use of tables of equivalent square fields for the derivation of the phantom scatter correction factor and phantom scatter related quantities, such as PDD and TPR, of rectangular fields is further discussed in Appendix 8.6 of this report. 
5. 


\section{Recommendations}

It is recommended to separate the total scatter correction factor $S_{c p}$ in two parts: the collimator scatter correction factor $S_{c}$ and the phantom scatter correction factor $S_{p}$. The use of $S_{c}$ and $S_{p}$ is recommended for calculations of treatment time and monitor units in clinical situations, as described in sections 2 and 3 .

$S_{c p}$ and $S_{c}$ are ratios of measured values, defined for each field size and normalized to unity for the reference field of $10 \mathrm{~cm} \times 10 \mathrm{~cm}$, the reference depth of $10 \mathrm{~cm}$ and the reference SSD (equal to SAD). This reference irradiation set-up should be the same, irrespective of the photon beam energy.

$S_{c}$ data have to be determined with a narrow cylindrical beam-coaxial phantom (mini-phantom) according to the method described in section 4. A description of the mini-phantom is given in Appendix 8.8. $\mathrm{S}_{c}$ factors have to be obtained for square and rectangular field sizes, with field sizes in the range used in clinical practice, i.e. from a minimum field size of $4 \mathrm{~cm} \times 4 \mathrm{~cm}$ or smaller, up to $40 \mathrm{~cm}$ $x 40 \mathrm{~cm}$. Data for rectangular fields can be obtained by direct measurement and/or by interpolation or data fitting methods. In this way, a full twodimensional table of $S_{\mathrm{c}}$ values has to be obtained.

$S_{c p}$ data have to be determined by using a full scatter water phantom, according to the method described in section $4 . S_{c p}$ values have to be measured for square fields. For consistency checks, the same measurements should be performed for a number of rectangular fields.

$S_{p}$ factors are derived with expression (2.1.3) from measured $S_{c p}$ and $S_{c}$ data for square field sizes. Results should be compared with published data; see section 5.3.1. Results of rectangular or arbitrarily shaped fields should be compared with interpolated values from the table, or with $S_{p}$ data taken for the equivalent square field ([8,9], see Appendices 8.4 and 8.6$)$.

Methods to reduce the number of measurements of $S_{c p}$ and $S_{c}$ in rectangular fields can be applied. Several references to methods for accurate data fitting are given in this report, see section 3.1.1 and Appendix 8.7. It is recommended, in these cases, to check and verify the product of $S_{c} \times S_{p}$ data for a number of elongated fields against direct measurement of $S_{c p}$. Differences between measured and calculated data should be within $1 \%$.

Reference wedge and tray transmission factors have to be determined for the reference irradiation set-up. Field size and SSD dependence of the wedge and tray factors have to be measured and accounted for in the relative wedge transmission factor and the relative tray transmission factor. The procedures are 
6.

described in section 3. Equations for calculation of monitor units given in section 2 should be replaced by those given in section 3 if beam modifiers are applied.

A summary of the measurements needed to obtain the data for the calculations is given in table 4.1 .

It is strongly recommended to compare the data found in the clinic with the data presented in this report. Note that especially $S_{c}$ data depend on the beam defining system and may vary considerably from one treatment machine to the other. $S_{c}$ data provided in the figures have to be considered as a first estimate. $S_{p}$ data are machine independent, but are uniquely related to the quality index of the beam. If the measured $S_{p}$ data differ from those presented in this report, further investigation of the dosimetry procedures is recommended. 
7.

\section{Acknowledgements}

The data presented in this report have been measured by physicists in a large number of radiotherapy departments in The Netherlands. Those who have contributed to this work are gratefully acknowledged by the members of the task group for their efforts, their helpful discussions and comments on the draft of this report and for making the measurement data available. 
7. 


\section{Appendices}

\subsection{Definition of scatter correction factors}

In this report, the total scatter correction factor $S_{c p}$ is defined as the absorbed dose per monitor unit, measured at the reference depth and the reference SSD for a specified collimator setting and a specified field size at the phantom surface, normalized to unity for the reference irradiation set-up:

$$
S_{c p}\left(v_{c}, v_{p}, d_{r e f}, f_{r e f}\right)=D\left(v_{c}, v_{p}, d_{r e f}, f_{r e f}\right) / D_{\text {ref }}
$$

Here, $v_{c}$ and $v_{p}$ express the field size at the SAD, defined by the collimator jaws, and the field size at the phantom surface, respectively. The reference irradiation set-up is defined as: a collimator setting yielding a $10 \mathrm{~cm} \times 10 \mathrm{~cm}$ field at the isocentre $\mathrm{V}_{\mathrm{c}, \text { rot }} ;$ a phantom depth equal to $\mathrm{d}_{\text {ref }}(=10 \mathrm{~cm})$; and an SSD equal to the SAD; the field size $v_{p, r e f}$ at the phantom surface is then numerically equal to $v_{c, r e f}$. From this definition it follows that $d_{\text {ref }}$ and $f_{\text {ref }}$ are fixed values and, therefore, $S_{c p}$ depends only on the variables $v_{c}$ and $v_{p}: S_{c p}\left(v_{c}, v_{p}\right)$.

A less strict definition is given by several other authors, and was also applied previously in reference [64], where $S_{c p}$ was deduced without defining $f_{\text {refr }}$

$$
\mathrm{S}_{\mathrm{cp}}\left(\mathrm{v}_{\mathrm{c}}, \mathrm{v}_{\mathrm{p}}, \mathrm{d}_{\mathrm{ref}}\right)=\mathrm{D}\left(\mathrm{v}_{\mathrm{c}}, \mathrm{v}_{\mathrm{p}}, \mathrm{d}_{\mathrm{ref}}\right) / \mathrm{D}\left(\mathrm{v}_{\mathrm{c}, \mathrm{rof},}, \mathrm{v}_{\mathrm{p}, \mathrm{re},}, \mathrm{d}_{\mathrm{ref}}\right)
$$

$S_{c p}$ is then written in those papers as:

$$
S_{c p}\left(v_{c}, v_{p}, d_{r e f}\right)=S_{c}\left(v_{c}, v_{p}, d_{r e f}\right) \times S_{p}\left(v_{c}, v_{p}, d_{r e f}\right)
$$

In the definitions of $S_{c p}$ given by equations (8.1.2) and (8.1.3) $d_{\text {ref }}$ is a variable and $f_{\text {ref }}$ is not specified. Both $d_{r e f}$ and $f_{\text {ref }}$ can be chosen freely by the user, e.g., at $d_{\max }, 5$ or $10 \mathrm{~cm}$ for $d_{\text {ref }}[34,64]$. Consistency in definition requires that $S_{c}$ data, measured for different but well-described phantom depths or SSD, should convert unambiguously into each other. This conversion is not a problem, as long as the phantom depth has been chosen beyond the range of the electron contamination [64]. However, difficulties arise when $S_{c}$ values are determined within the range of the electron contamination, i.e. when $S_{c}$ values are measured at shallow depths, see Appendix 8.2.

A second problem arises in the conversion, if $S_{c p}$ has been defined for SSD values not equal to the SAD. In that situation the requirements with respect to $v_{c, \text { ref }}$ and $v_{p, \text { ref }}$ can not be fulfilled simultaneously. A choice between both reference field sizes has to be made and redefinition is then needed.

In conclusion, the mutual relation between $S_{c}$ data, determined for different but well-described phantom depths and SSDs, is essentially a part of the $S_{c p}$ 
8.

definition according to equation (8.1.2), to maintain the consistency of that definition. The $S_{c p}$ definition according to equation (8.1.1) is inherently consistent due to the requirement that $D\left(v_{c}, v_{p}, d_{\text {ref } r} f_{\text {ref }}\right)$ and $D_{\text {ref }}$ have to be determined under the same conditions, i.e. $S_{c p}$ is defined for only a single phantom depth and SSD. Conversion problems of $S_{c p}$ values, defined according to equation (8.1.2), to dose values at other phantom depths or SSDs remain, therefore, part of the dose calculation algorithms. The more restrictive $S_{c p}$ definition in equation (8.1.1) is preferred over the one in equation (8.1.2). 
8.

\subsection{Scatter correction factors at other reference depths}

Within the context of this report $S_{c}, S_{p}$ and $S_{c p}$ values have been strictly defined for a reference irradiation set-up (Appendix 8.1). However, the reference values are a particular choice and these conventions may differ in principle from one radiotherapy department to another. Different definitions of the scatter correction factors found in the literature or required for a treatment planning system, can be reasons to deviate from what is adopted here. A comparison of scatter correction factors measured in different radiotherapy departments might be helpful in quality control programs. A conversion is then required. The reference field size is generally taken equal to $10 \mathrm{~cm} \times 10 \mathrm{~cm}$ and the reference SSD equal to SAD. Conversion methods for other reference field sizes are therefore considered to be irrelevant; for other SSDs an example is given in Appendix 8.3. Differences in the choice of the reference depth are most likely to occur.

Two practical situations can now be distinguished: the reference depth, which should be taken equal for field sizes $v$ and $v_{\text {ref }}$ (see figure 8.1), is defined:

a) beyond the range of electron contamination, but different from the reference depth of $10 \mathrm{~cm}$ given in this report;

b) within the range of electron contamination.

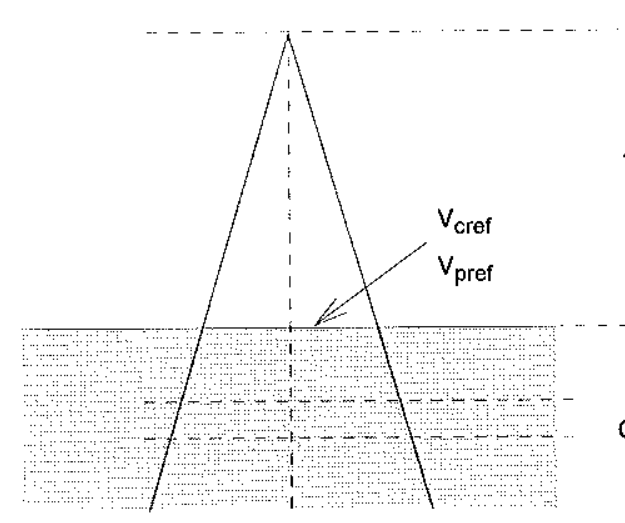

a)

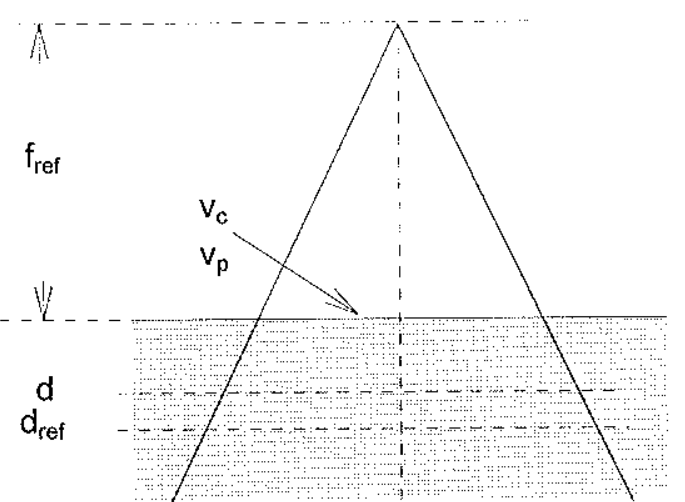

b)

Figure 8.1 Two dose points, one at the reference depth, $d_{r e f}$, and one at the new depth, d. a) in the reference field, $v_{\text {ref }} ;$ b) in an arbitrary field, $v$; both with a source-surface distance equal to $f_{\text {ter }}$. 


\section{Situation a: beyond the range of the electron contamination}

For this situation, the $S_{c}$ definition is basically identical to the one recommended in this report, as long as the SSD is equal to SAD. The conversion can be obtained using equation (2.3.1):

$$
S_{c}\left(v_{c}, d\right) \times S_{p}\left(v_{p}, d\right)=D\left(v_{c}, v_{p}, d, f_{r e f}\right) / D\left(v_{c, r e f}, v_{p, \text { ref }}, d, f_{r e f}\right)
$$

in which the phantom depth, $d$, is now introduced as a variable. The value of $d$ can be defined by the user, e.g. $10 \mathrm{~cm}$, as is done in this report. As discussed in section 2 and shown by van Gasteren et al. [64], in this case the magnitude of $S_{c}$ does not depend on the phantom depth, i.e.:

$$
S_{c}\left(v_{c}, d\right)=S_{c}\left(v_{c}, d_{r e f}\right)
$$

Both dose values in equation (8.2.1) can be rewritten to dose values at other phantom depths by:

$$
D\left(v_{c}, v_{p}, d, f_{r e f}\right)=D\left(v_{c}, v_{p}, d_{r e f}, f_{r e f}\right) \times R D D\left(v_{c}, v_{p}, d, f_{r e f}\right)
$$

By combining equations (8.2.1) to $(8.2 .3), \mathrm{S}_{\mathrm{p}}$ can be written as [65]:

$$
S_{p}\left(v_{p}, d\right)=S_{p}\left(v_{p}, d_{r e f}\right) \times R D D\left(v_{c}, v_{p}, d, f_{r e f}\right) / R D D\left(v_{c, r e f}, v_{p, r e f}, d, f_{r e f}\right)
$$

Equations (8.2.2) and (8.2.4) can be applied without any limitation, as long as the phantom depths have been chosen beyond the range of electron contamination.

\section{Situation b: within the range of the electron contamination}

If measurements of $S_{c}$ are performed in a radiotherapy department at shallow depths $d$, the resulting values, $S_{c}\left(v_{c}, d\right)$, will include the dose contribution of the electron contamination. In that case, equation (8.2.2) is no longer valid. As discussed in Appendix 8.1, a conversion from $S_{c}\left(v_{c}, d\right)$ to $S_{c}\left(v_{c}, d_{r e f}\right)$ or vice versa, would require an exact knowledge of the dose contribution of the electron contamination as a function of field size and depth. Algorithms calculating that conversion are not readily available (e.g., $[5,6,71]$ ) and conversion has, therefore, to be done based on measurements. It is easy to show that the ratio of measured $S_{c}\left(v_{c}, d\right)$ and $S_{c}\left(v_{c}, d_{r e f}\right)$ values just yields that conversion factor, $S_{c, \text { el }}\left(v_{c}, d\right)$, for that field size and depth [72]. $S_{c, \text { el }}\left(V_{c}, d\right)$ can thus be considered as a factor which describes the contribution of the electron contamination to the collimator scatter correction factor if measured at shallow depths.

In summary, the conversion of collimator scatter correction factors measured at phantom depths beyond the range of contamination, to a value at shallow depth is not easy to perform. Therefore, it is strongly recommended to determine and use the collimator scatter correction factor $\mathrm{S}_{\mathrm{c}}$ only with its definition at the reference depth of $10 \mathrm{~cm}$. 


\subsection{Relations between the quantities in the fixed SSD and the isocentric formalism}

$S_{c p}$ is defined in this report as a function of the field size at the phantom surface for a specifically described geometry: the fixed SSD system. Another possibility would have been to define and measure $S_{c p}$ in such a way that the distance between source and measuring point, $f+d$, equals SAD: the isocentric approach. There are no physical arguments supporting the choice for either one of these methods. The choice of the task group for the fixed SSD approach as a starting point in this report, is lead by the fact that measurements are generally performed within such a geometry with the surface of the full scatter water phantom and the mini-phantom placed at a distance equal to SAD. In this approach there is no need to change water levels. Measurements are performed very straightforward, and results can be used directly in the fixed SSD calculation system. In many radiotherapy departments measurements are performed in such a way and, if necessary, TPR data for use in isocentric calculations are derived by using conversion rules. For example, the conversion rules given by Burns [8] transform PDD and peak scatter factor data into TPR data, and vice versa. It is sometimes usefull to measure directly in an isocentric set-up [18], but then also conversion rules are necessary to enable fixed SSD calculations.

This appendix discusses the geometries and definitions of both the fixed SSD approach and the isocentric approach and shows what the relation is between the corresponding quantities in these two approaches [71].

\section{The fixed SSD formalism}

In this situation, the quantities percentage depth dose PDD and phantom scatter correction factor $S_{p}$ are defined as a function of field size at the phantom surface, while $S_{c}$ is defined by the collimator setting at SAD. In the reference situation, in which the basic data are measured, the source-surface distance is set at $f_{\text {ref }}$. PDDs, $S_{c}$ and $S_{p}$ are measured as a function of field size at $f_{\text {ref }}$. The ionization chamber is placed at $f_{\text {ref }}+d_{\text {ref }}(=100+10 \mathrm{~cm})$.

\section{The isocentric formalism}

In this situation, the quantities tissue-phantom ratio TPR, collimator scatter correction factor $S_{c}{ }_{c}^{\text {iso }}$ and phantom scatter correction factor $S_{p}{ }^{\text {iso }}$ are given as a function of field size at SAD. Note that the notation $S_{c}$ iso,$S_{p}$ iso,$S_{c p}$ iso and $D_{\text {ref }}$ iso is used consistently for the quantities in the isocentric formalism. In the reference situation, in which the basic data are measured, the source-to-point distance is now fixed, while the source-surface distance is variable and depends on the depth of the measuring point in the phantom. $S_{c}$ iso and $S_{p}$ iso are measured as a function of field size at SAD, while the depth is equal to $d_{\text {ref }}$. 
8.

Consequently, definitions and measurement geometries are essentially different for the fixed SSD and the isocentric formalism. Data obtained in one irradiation set-up should therefore not be confused with those obtained in the other. A summary of the definitions is given in table 8.1 .

Table 8.1 Summary of the definitions of the reference irradiation set-up as used in the fixed SSD and the isocentric formalism. It is important to note that in both formalisms the same $d_{\text {ref }}$ is used.

\begin{tabular}{|c|c|c|}
\hline Quantity & Fixed SSD formalism & Isocentric formalism \\
\hline reference depth: & $d_{\text {ref }}=10 \mathrm{~cm}$ & $d_{r e f}=10 \mathrm{~cm}$ \\
\hline $\begin{array}{l}\text { source-surface } \\
\text { distance (for linacs): }\end{array}$ & $f=f_{r e t}=100 \mathrm{~cm}$ & $f=f_{r e f}-d_{r e t}=90 \mathrm{~cm}$ \\
\hline $\begin{array}{l}\text { field size related to } \\
\text { collimator scatter: }\end{array}$ & $v_{c^{\prime}}$ defined at $f_{r e f}$ & $v_{c^{\prime}}$ defined at $f_{r e f}$ \\
\hline $\begin{array}{l}\text { field size related to } \\
\text { phantom scatter: }\end{array}$ & $v_{p}$ defined at surface at $f$ & $v_{\text {ref }}\left(d_{\text {ret }}\right)$, defined at depth $d_{\text {ref }}$ \\
\hline $\begin{array}{l}\text { field size at } \\
\text { reference depth: }\end{array}$ & $(110 / 100) \times v_{p}$ & $v(d)$ \\
\hline $\begin{array}{l}\text { field size at } \\
\text { phantom surface: }\end{array}$ & $v_{p}$ & $(90 / 100) \times v(d)$ \\
\hline $\begin{array}{l}\text { dose per } M U \text { at the } \\
\text { reference point: }\end{array}$ & $D_{r e f}=D\left(v_{c, r e f}, v_{p, r e f}, d_{r e f t}, f_{r e f}\right)$ & 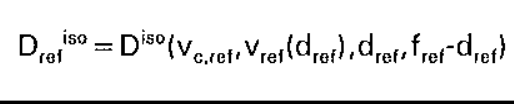 \\
\hline
\end{tabular}

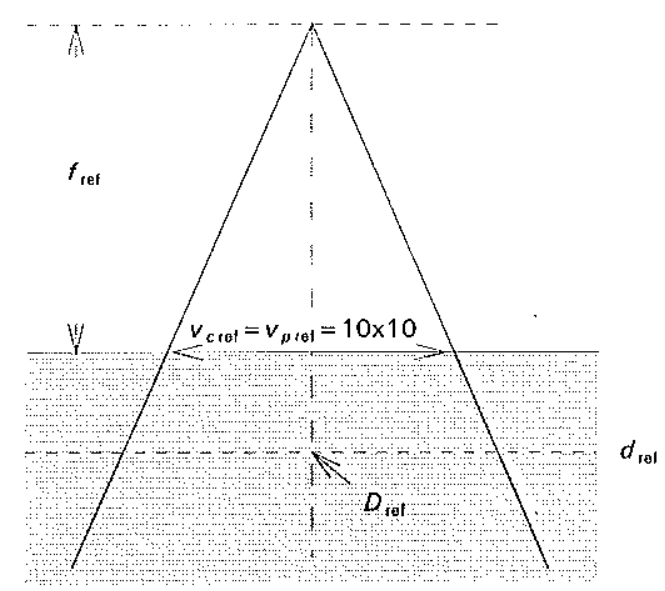

fixed SSD set-up

a)

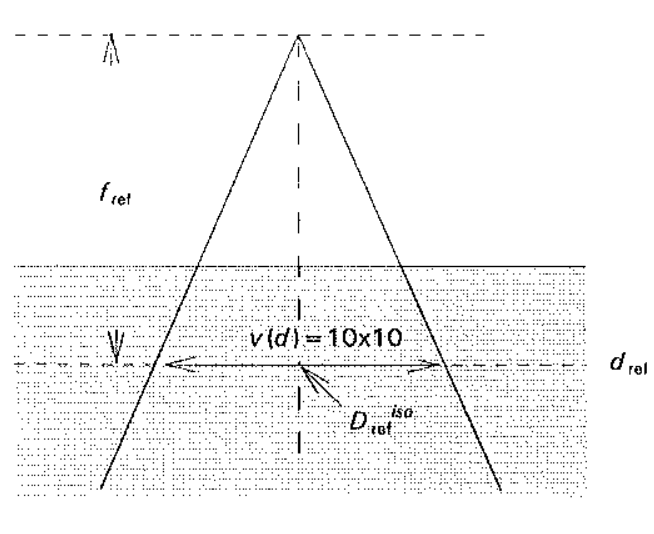

isocentric set-up

b)

Figure 8.2 Reference conditions for both the fixed SSD (a) and the isocentric (b) formalism. 
8.

The relation between $D_{\text {ref }}$ and $D_{\text {ref }}^{\text {iso }}$

Using the numerical data of table 8.1 for treatment units with $f_{\text {ref }}=100 \mathrm{~cm}$, the situation in the isocentric set-up of figure 8.2.b can be described with the parameters from the fixed SSD formalism. The reference dose $D_{\text {ref }}$ iso is then given by:

$$
D_{\text {ref }}^{\text {iso }}=D_{\text {ref }} \times\left\{\left(f_{\text {ref }}+d_{\text {ref }}\right) / f_{\text {ref }}\right\}^{2} \times S_{p}\left\{\left(f_{\text {ref }} /\left(f_{\text {ref }}+d_{\text {ref }}\right) / v_{p, r e f}\right\}\right.
$$

or, more specifically, by using the reference values:

$$
D_{\text {ref }}^{\text {iso }}=D_{\text {ref }} \times 1.21 \times S_{p}(9.1)
$$

where $S_{p}(9.1)$ is the phantom scatter correction factor, in the fixed SSD formalism, of a square $9.1 \mathrm{~cm} \times 9.1 \mathrm{~cm}$ field.

In the same way, the situation in the fixed SSD set-up of figure 8.2.a can be described using the parameters from the isocentric formalism, where the reference dose $D_{\text {ref }}$ is given by:

$$
D_{\text {ref }}=D_{\text {ref }}^{\text {iso }} \times\left\{f_{\text {ref }} /\left(f_{\text {ref }}+d_{r e f}\right)\right\}^{2} \times S_{p}^{\text {iso }}\left\{\left(\left(f_{\text {ref }}+d_{r e f}\right) / f_{\text {ref }}\right) v_{\text {ref }}\left(d_{\text {ref }}\right)\right\}
$$

and, with the reference values:

$$
D_{\text {ref }}=D_{\text {ref }}^{\text {iso }} \times 0.826 \times S_{p}^{\text {iso }}(11)
$$

\section{Calculation of $S_{c}^{\text {iso }}$ from $S_{c}$ data, and vice versa}

Comparison of the isocentric situations described above with those of the fixed SSD formalism, shows that the collimator scatter correction factor is identical, provided the same normalization is applied, i.e.:

$$
\mathrm{S}_{\mathrm{c}}\left(\mathrm{V}_{\mathrm{c}}\right)=\mathrm{S}_{\mathrm{c}}^{\text {iso }}\left(\mathrm{V}_{\mathrm{c}}\right)
$$

with $v_{c, r e f}=10 \mathrm{~cm} \times 10 \mathrm{~cm}$ at $f_{\text {ref }}=S A D$.

\section{Calculation of $S_{p}{ }^{\text {iso }}$ from $S_{p}$ data}

It is assumed that in the isocentric approach field sizes are taken at $S A D=100$ $\mathrm{cm}$, at a phantom depth of $10 \mathrm{~cm}, v\left(d_{\text {ref }}\right)$. According to Johns et al. [32], the relative phantom scatter contribution in a $10 \mathrm{~cm} \times 10 \mathrm{~cm}$ (TPR) field is identical to that found in the (PDD) field with the size $9.1 \mathrm{~cm} \times 9.1 \mathrm{~cm}$ at the phantom surface at $S S D=100 \mathrm{~cm}$. It has to be taken into account that values of $S_{p}$ iso are normalized to unity for the field $10 \mathrm{~cm} \times 10 \mathrm{~cm}$ at SAD. In this case one finds $S_{p}$ iso values from $S_{p}$ values with (see tables 5.2 and 5.3):

$$
S_{p}^{\text {iso }}\left(v\left(d_{\text {ref }}\right)\right)=S_{p}\left\{\left(f_{\text {ref }} /\left(f_{\text {ref }}+d_{\text {ref }}\right)\right) v\left(d_{\text {ref }}\right)\right\} / S_{p}\left\{\left(f_{r e f} /\left(f_{\text {ref }}+d_{\text {ref }}\right)\right) v_{\text {ref }}\left(d_{\text {ref }}\right)\right\}
$$


8.

or, by using the reference values:

$$
S_{p}^{\text {iso }}\left(v\left(d_{r e f}\right)\right)=S_{p}\left(0.91 v\left(d_{r e f}\right)\right) / S_{p}(9.1)
$$

\section{Calculation of $S_{p}$ from $S_{p}^{\text {iso }}$ data}

In the same way we need to consider the fixed SSD situation from the point of view of the isocentric situation, with a reference (PDD-) field of $10 \mathrm{~cm} \times 10 \mathrm{~cm}$ at the phantom surface corresponding to a $11 \mathrm{~cm} \times 11 \mathrm{~cm}$ (TPR-) field. Again, the normalization at $d_{\text {ref }}=10 \mathrm{~cm}$ has to be taken into account. So, using the present definitions, $S_{p}$ data are calculated from $S_{p}$ iso data with:

$$
S_{p}\left(v_{p}\right)=S_{p}{ }_{p}^{\text {iso }}\left\{\left(\left(f_{\text {ref }}+d_{\text {ref }}\right) / f_{\text {ref }}\right) v_{p}\right\} / S_{p}{ }^{\text {iso }}\left\{\left(\left(f_{\text {ref }}+d_{\text {ref }}\right) / f_{\text {ref }}\right) / v_{p, \text { ret }}\right\}
$$

Or, by using the reference values:

$$
S_{p}\left(v_{p}\right)=S_{p}^{\text {iso }}\left(1.1 v_{p}\right) / S_{p}^{\text {iso }}(11)
$$

\section{Relation between TPR, $S_{p}$ and $R D D$}

It can be derived that the following expression relates TPR, written as a function of $v(d)$, i.e. the field size defined at the depth $d$, to $S_{p}$ and RDD [71]:

$$
\begin{gathered}
\operatorname{TPR}(v(d), d)=\frac{S_{p}\left\{\left(f_{r e f} /\left(f_{r e f}+d\right)\right) v(d)\right\}}{S_{p}\left\{\left(f_{r e f} /\left(f_{r e f}+d_{r e f}\right)\right) v(d)\right\}} \times\left\{\left(f_{r e f}+d\right) /\left(f_{r e f}+d_{r e f}\right)\right\}^{2} \\
\quad \times \operatorname{RDD}\left\{\left(f_{r e f} /\left(f_{r e f}+d\right)\right) v(d), d, f_{r e f}\right\}
\end{gathered}
$$

In this way TPRs can be calculated using existing RDD (or PDD) and $\mathrm{S}_{\mathrm{p}}$ data.

The relation between $R D D, S_{p}^{i s o}$ and TPR

We can now use equations (8.3.8) and (8.3.10) to find the relation of the relative depth dose RDD with the phantom scatter correction factor $S_{p}$ iso and TPR. When the appropriate field size relationships are taken into account, it can be found that:

$$
\begin{aligned}
& \operatorname{RDD}\left(v_{p}, d, f_{\text {ref }}\right)=\frac{S_{p}^{\text {iso }}\left\{\left(\left(f_{\text {ref }}+d\right) / f_{\text {ref }}\right) v_{p}\right\}}{S_{p}^{\text {iso }}\left\{\left(\left(f_{\text {ref }}+d_{\text {ref }}\right) / f_{\text {ref }}\right) / v_{p}\right\}} \times\left\{\left(f_{\text {ref }}+d_{r e f}\right) /\left(f_{\text {ref }}+d\right)\right\}^{2} \\
& x \operatorname{TPR}\left\{\left(\left(f_{r e f}+d\right) / f_{r e f}\right) v_{p}, d\right\}
\end{aligned}
$$

In this way, RDDs (and PDDs) can be calculated using existing TPR and $\mathrm{S}_{\mathrm{p}}$ iso data. 


\section{4 $S_{p}$ and PDD (and RDD) for arbitrarily shaped fields}

According to equation (2.3.2), the dose $D$ in a phantom at depth $d$ at the beam axis with collimator field size $v_{c}$, phantom surface field size $v_{p}$, and a sourcesurface distance equal to $S A D$, is written as:

$$
D\left(v_{c}, v_{p}, d, f_{r e f}\right)=D_{r e f} \times S_{c}\left(v_{c}\right) \times S_{p}\left(v_{p}\right) \times R D D\left(v_{p}, d, f_{r e f}\right)
$$

In the situation that rectangular or blocked fields are used, $S_{c}$ and $S_{p}$ have to be determined for that specific geometry. $S_{c}$ can to be determined by measurement; see section 2.1 and Appendix 8.5. However, $S_{p}$ can also be derived from $S_{p}$ values tabulated for square field sizes. $S_{p}$ values of square fields should then be transformed into $S_{p}$ values of equivalent circular fields, e.g. by using the method described by Day and Aird [8,9], see also Appendix 8.7. Then, when we subdivide the arbitrarily shaped field $v_{p}$ into small sectors with angle $\phi$, which each contribute to the scatter dose, we may integrate over these sectors [13]:

$$
\begin{aligned}
D\left(v_{c}, v_{p}, d\right) & =(1 / 2 \pi)_{0}^{2 \pi} \int D\left(v_{c}, v_{p}(\phi), d\right) \cdot d \phi \\
& =(1 / 2 \pi) \times D_{r e f} \times S_{c}\left(v_{c}\right) \times{ }_{0}^{2 \pi} \int S_{p}\left(v_{p}(\phi)\right) R D D\left(v_{p}(\phi), d\right) \cdot d \phi
\end{aligned}
$$

This means that the total scatter contribution $S_{p}\left(v_{p}\right)$ is the result of a summation of the scatter contribution of the small sectors. So:

$$
S_{p}\left(v_{p}\right) \times \operatorname{RDD}\left(v_{p}, d\right)=(1 / 2 \pi) \times x_{0}^{2 \pi} \int S_{p}\left(v_{p}(\phi)\right) \operatorname{RDD}\left(v_{p}(\phi), d\right) \cdot d \phi
$$

i.e. not $\operatorname{RDD}\left(v_{p}, d\right)$ itself, but the product $S_{p}\left(v_{p}\right) \times \operatorname{RDD}\left(v_{p}, d\right)$ is the quantity of interest. In the calculation of RDD of an arbitrarily shaped field at an arbitrary depth, $S_{p}$ must be used as a weighting function.

Both equation (8.4.2) and equation (8.4.3) can be simplified if $\mathrm{d}$ has been taken equal to $d_{r e f}$. In that case, RDD is equal to unity and $(8.4 .3)$ is reduced to:

$$
S_{p}\left(v_{p}\right)=(1 / 2 \pi) \times x_{0}^{2 \pi} \int S_{p}\left(v_{p}(\phi)\right) \cdot d \phi
$$

This relationship may be used to calculate $S_{p}$ data of arbitrarily shaped fields, i.e. for rectangular and blocked fields, using a suitable set of $S_{p}$ data for equivalent circular fields.

\footnotetext{
${ }^{1}$ Because $f_{\text {ref }}$ is not relevant in the expressions of this paragraph, it has been omitted.
} 


\section{5 $\mathrm{S}_{\mathrm{c}}$ for partially blocked fields}

In clinical routine the $S_{c}$ value of a partially blocked irradiation field, $S_{c, b l o c k}$, can be taken equal to the $S_{c}$ value of the field size set by the collimator jaws $S_{c}\left(v_{c}\right)$ as long as the amount of photons scattered from the primary collimator and the flattening filter, as observed from the point of interest, is limited by the collimator jaws and not by the additional blocks on the tray $[41,45,60,63]$. For these situations, $S_{c, b l o c k}$ values can be found from $S_{c}$ data which are already available for rectangular fields. In other situations, $S_{c, \text { block }}$ has to be determined experimentally. The decision whether or not the $S_{c, b l o c k}$ value has to be determined by measurement, depends on the construction of the head of the treatment machine and the distance between the focus and the point of interest (see figures 3.1 and 8.3.a). Based on the specific geometry, a decision criterion can be developed.

In figure 8.3.b, the rectangular field size, set-up by the collimator jaws at SAD, has been divided into four quadrants by the two cross-wires. For each quadrant it has to be determined whether the additional block or the collimator hides the flattening filter from the point of view at depth $d$. In the situation that the additional block is the limiting factor in one of these quadrants, $S_{c}$ has to be determined by measurement. Using figure 8.3.b, the next criterion whether the block or the collimator jaw hides the filter has been derived for the $\mathrm{X}$-collimator jaws:
$\left.b_{x} \mid \cos (\theta)\right) \mid C_{x} /(X / 2)$
$<1: \quad \mathrm{S}_{\mathrm{c}, \text { block }}$ has to be determined, in
principle, by measurement;
$\left.b_{x} \mid \cos (\theta)\right) \mid C_{x} /(X / 2)$
$\geq 1: \quad S_{c, b l o c k}$ can be taken equal to $S_{c}\left(v_{c}\right)$.

In this criterion (see figure 8.3.a):

- $b_{x}$ is equal to the minimum distance between the block edge and the central photon beam axis, determined at the isocentre, i.e. at SAD;

- $\theta$ is equal to the angle between $b_{x}$ and the $X$-axis;

- $C_{x}=\frac{\left(f+d-U_{x}\right) T}{(f+d-T) U_{x}}$

where $d$ is the depth in the phantom, $T$ is the distance from the focus to the additional shielding blocks and $U_{x}$ is the distance from the focus to the $X$ collimator jaw. 
8.

- $\mathrm{X} / 2$ is equal to the position of the $\mathrm{X}$-collimator jaw at the isocentre; i.e. half the side of a symmetrical field with setting $X$.

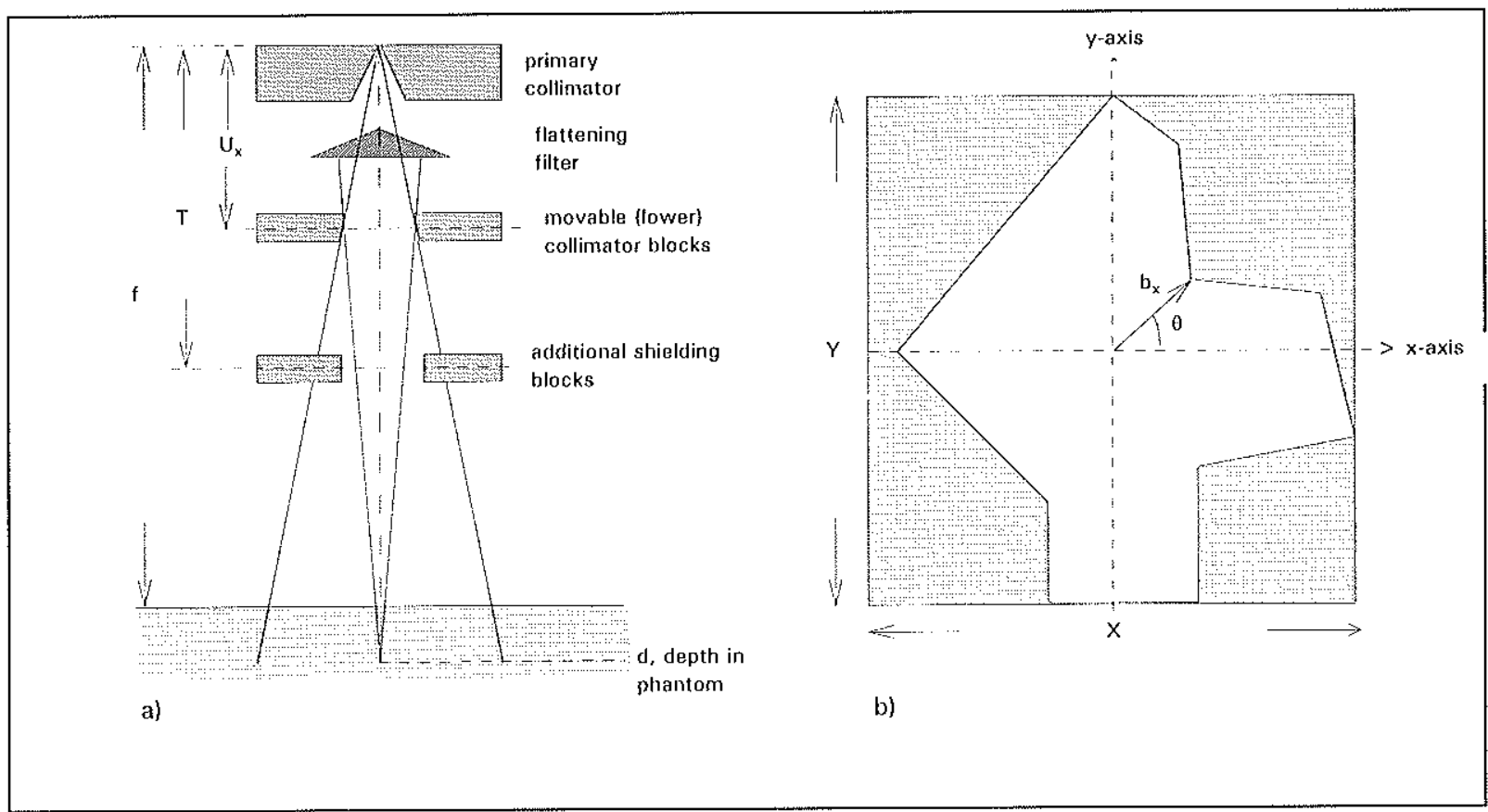

Figure 8.3 Definition of quantities used in the decision rule whether or not $\mathrm{S}_{\mathrm{c}, \text { block }}$ can be taken equal to $S_{c}$. a) Side view of the beam; b) top view of blocked field at SAD.

In clinical situations several methods can be used to deduce $b_{x}$ either with $a$ simple computer program or estimated by eye.

For the $Y$-collimator jaws a similar expression can be used, replacing the indices $\mathrm{x}$ by $\mathrm{y},|\cos (\theta)|$ by $|\sin (\theta)|$, and the variable $X$ by $Y$. The $U_{x}, U_{y}$ and $T$ values can be found from the details of the treatment machine from the manufacturer.

As an example, these values have been presented for one type of treatment machine in table 8.2, with $f$ equal to $S A D$ and the phantom depth $d$ equal to $d_{\text {ref }}$. Finally, two numerical examples are given. 
Table $8.2 \mathrm{C}$ values, required for the decision criterion whether or not to measure $S_{c}$ values.

\begin{tabular}{l|lllllll}
\hline Manufacturer & Type & $U_{x}(\mathrm{~cm})$ & $U_{y}(\mathrm{~cm})$ & $T(\mathrm{~cm})$ & $\begin{array}{l}S A D \\
(\mathrm{~cm})\end{array}$ & $C_{x}$ & $C_{y}$ \\
\hline Philips & $\mathrm{SL25}$ & 44 & 34 & 67 & 100 & 2.34 & 3.48 \\
\hline
\end{tabular}

\section{Examples}

For the Philips SL25 treatment unit, the following data are derived, using the values of table 8.2 :

A field size of $20 \mathrm{~cm} \times 20 \mathrm{~cm}$ is defined at the isocentre; reduced by blocks on the tray to a field of $10 \mathrm{~cm} \times 10 \mathrm{~cm}$. Then: $b_{x} C_{x} /(X / 2)=1.17$ (resp. 1.74 for the $Y$ collimator setting). Conclusion: $S_{c, \text { btock }}=S_{c}(20)$.

A field size of $20 \mathrm{~cm} \times 20 \mathrm{~cm}$ is defined at the isocentre; reduced by blocks on the tray to a field of $5 \mathrm{~cm} \times 5 \mathrm{~cm}$. Then: $b_{x} C_{x} /(X / 2)=0.585$ (resp. 0.87 for the $\mathrm{Y}$ collimator setting). Conc/usion: $\mathrm{S}_{\mathrm{c}, \text { block }} \neq \mathrm{S}_{c}(20)$, and needs to be measured. 


\subsection{The concept of equivalent fields}

The use of BJR Supp. 17 tables [8] of equivalent square fields for dose calculations is widespread. The revised version, BJR Supp. 25 [9], contains a more thorough discussion on this topic, but without changes in the contents of the tables. The equivalent square field is defined in BJR Supp. 25 as "that standard square field which has the same central axis depth dose characteristics as the non-standard field". This method was developed at a time when beam data were measured and applied in calculations, based on PDD, BSF, PSF and $d_{m}$. The current philosophy in calculation methods, however, is based on data defined and measured at a reference depth $\mathrm{d}_{\mathrm{ref}}=10 \mathrm{~cm}$, on a separation of phantom and collimator scatter and on the use of the relative depth dose, RDD. In this concept, problems related to the influence of contaminating electrons in the beam at shallow depths are eliminated. If the reference conditions are changed, it is not a priori evident that the tools for calculating the dose, such as a table of equivalent square fields, remain unchanged. In principle, separate equivalent square fields may be necessary for the derivation of the phantom scatter factor and for the phantom scatter related quantities such as PDD, TAR and TPR of a rectangular or irregular field on the one hand, and for the collimator scatter factor on the other hand.

\section{Phantom scatter}

The equivalent square field, to be used for the determination of the phantom scatter factor and phantom scatter related quantities, is defined here as the square field which has the same phantom scatter contribution at the reference point in the beam: at $10 \mathrm{~cm}$ depth on the central axis, as the arbitrarily shaped field under consideration.

The use of the BJR Supp. 25 tables of equivalent square fields in relation to phantom scatter was recently discussed by Venselaar et al. [70]. In their analysis, energy-specific tables of equivalent squares for phantom scatter were derived for 4 beam qualities, based on $S_{p}$ data included in this report and covering the range of $\mathrm{Ql}$ of 0.573 to $0.783\left({ }^{60} \mathrm{Co}\right.$ to $\left.25 \mathrm{MV}\right)$. It was shown that the use of the energy-specific tables could eventually lead to a difference of $0.5-1.0 \%$ in the value of $S_{p}$, compared to the use of the BJR-table, in which the use of the BJRtable systematically leads to a lower value of $S_{p}$. The relatively small differences between the 4 energy-specific tables mutually allowed the construction of a new average table of equivalent square fields. When this average table is applied instead of the BJR-table, improvements were obtained, especially in the median beam qualities, for example the 6 and $10 \mathrm{MV}$ beams. For the highest energy, 25 $\mathrm{MV}$, the benefit is less, while for ${ }^{60} \mathrm{Co}$ no improvements were found.

Because of the relatively small deviations in the $S_{p}$ values and because of the widespread use of the BJR-table, its continued use is considered justified for clinical routine. However, it was concluded that a higher accuracy in dose 
8.

calculation can indeed be obtained by the application of energy-specific tables of equivalent square fields for phantom scatter, or by using the average table discussed above. In cases where optimal accuracy is needed, it is necessary to pay attention to all aspects of the dose calculation procedures. Then, the use of the most accurate table of equivalent square fields is recommended. The new average table 8.3 can fulfil this recommendation and is therefore presented in this report.

Table 8.3 Table of equivalent square fields for the derivation of phantom scatter factors and phantom scatter related quantities for rectangular fields with field sides $s 1 \times s 2$. The new table was constructed by averaging 4 energy-specific tables for ${ }^{60} \mathrm{Co}, 6,10$ and $25 \mathrm{MV}$ photon beams. Dimensions are in $\mathrm{cm}$. See [70] for more details.

\begin{tabular}{|c|c|c|c|c|c|c|c|c|c|c|c|c|c|c|c|c|c|c|c|c|}
\hline$s 1 \mid s 2$ & 2.0 & 4.0 & 6.0 & 8.0 & 10.0 & 12.0 & 14.0 & 16.0 & 18.0 & 20.0 & 22.0 & 24.0 & 26.0 & 28.0 & 30.0 & 32.0 & 34.0 & 36.0 & 38.0 & 40.0 \\
\hline 2.0 & 2.0 & & & & & & & & & & & & & & & & & & & \\
\hline 4.0 & 2.8 & 4.0 & & & & & & & & & & & & & & & & & & \\
\hline 6.0 & 3.3 & 4.9 & 6.0 & & & & & & & & & & & & & & & & & \\
\hline 8.0 & 3.6 & 5.4 & 6.9 & 8.0 & & & & & & & & & & & & & & & & \\
\hline 10.0 & 3.7 & 5.7 & 7.4 & 8.8 & 10.0 & & & & & & & & & & & & & & & \\
\hline 12.0 & 3.8 & 5.9 & 7.7 & 9.4 & 10.9 & 12.0 & & & & & & & & & & & & & & \\
\hline 14.0 & 3.9 & 6.0 & 7.9 & 9.9 & 11.6 & 12.9 & 14.0 & & & & & & & & & & & & & \\
\hline 16.0 & 4.0 & 6.1 & 8.1 & 10.3 & 12.2 & 13.8 & 15.0 & 16.0 & & & & & & & & & & & & \\
\hline 18.0 & 4.0 & 6.2 & 8.3 & 10.6 & 12.7 & 14.5 & 15.9 & 17.1 & 18.0 & & & & & & & & & & & \\
\hline 20.0 & 4.0 & 6.2 & 8.5 & 10.9 & 13.2 & 15.1 & 16.6 & 18.0 & 19.1 & 20.0 & & & & & & & & & & \\
\hline 22.0 & 4.0 & 6.3 & 8.6 & 11.2 & 13.7 & 15.7 & 17.3 & 18.7 & 20.0 & 21.1 & 22.0 & & & & & & & & & \\
\hline 24.0 & 4.1 & 6.4 & 8.7 & 11.5 & 14.1 & 16.1 & 17.9 & 19.4 & 20.7 & 22.0 & 23.1 & 24.0 & & & & & & & & \\
\hline 26.0 & 4.1 & 6.4 & 8.8 & 11.7 & 14.4 & 16.6 & 18.4 & 19.9 & 21.4 & 22.7 & 24.0 & 25.1 & 26.0 & & & & & & & \\
\hline 28.0 & 4.1 & 6.4 & 8.9 & 11.9 & 14.7 & 16.9 & 18.8 & 20.4 & 22.0 & 23.4 & 24.7 & 26.0 & 27.1 & 28.0 & & & & & & \\
\hline 30.0 & 4.1 & 6.5 & 9.0 & 12.0 & 14.9 & 17.2 & 19.1 & 20.9 & 22.5 & 24.0 & 25.4 & 26.7 & 28.0 & 29.1 & 30.0 & & & & & \\
\hline 32.0 & 4.1 & 6.5 & 9.1 & 12.2 & 15.1 & 17.5 & 19.4 & 21.2 & 22.8 & 24.4 & 25.9 & 27.3 & 28.7 & 29.9 & 31.0 & 32.0 & & & & \\
\hline 34.0 & 4.1 & 6.5 & 9.1 & 12.3 & 15.3 & 17.7 & 19.7 & 21.5 & 23.2 & 24.8 & 26.4 & 27.9 & 29.3 & 30.6 & 31.9 & 33.0 & 34.0 & & & \\
\hline 36.0 & 4.1 & 6.5 & 9.1 & 12.4 & 15.4 & 17.8 & 19.9 & 21.7 & 23.4 & 25.1 & 26.7 & 28.3 & 29.8 & 31.2 & 32.6 & 33.8 & 35.0 & 36.0 & & \\
\hline 38.0 & 4.1 & 6.5 & 9.2 & 12.5 & 15.5 & 17.9 & 20.0 & 21.9 & 23.7 & 25.3 & 27.0 & 28.7 & 30.2 & 31.7 & 33.2 & 34.6 & 35.8 & 36.9 & 38.0 & \\
\hline 40.0 & 4.1 & 6.5 & 9.2 & 12.5 & 15.6 & 18.1 & 20.1 & 22.0 & 23.8 & 25.6 & 27.3 & 28.9 & 30.5 & 32.1 & 33.6 & 35.1 & 36.5 & 37.8 & 39.0 & 40.0 \\
\hline
\end{tabular}

\section{Collimator scatter}

In exactly the same way, the equivalent square field to be used for the determination of the collimator scatter factor, is defined here as the square field that has the same collimator scatter contribution to the reference point in the beam at $10 \mathrm{~cm}$ depth at the central axis, as the arbitrarily shaped field under consideration.

The same approach as describe above for phantom scatter, can be applied to determine a full table of equivalent square fields from the measured collimator scatter data of a specific photon beam. This should be done with caution, because the resulting table will be typically machine dependent and should incorporate the collimator exchange effect (CEE). Some papers report differences in collimator 
8.

scatter with $X-Y$ vs. $Y-X$ setting of up to $6 \%[44,57]$, although others report smaller differences (2\%, see for example section 5.2 .2 of this report). It is therefore, in all cases, recommended to measure the collimator scatter factors for each individual treatment machine and photon beam quality.

The use of a 2-D table of $S_{c}$ values is considered to be relatively simple in practice with present-day computer technology. Parametrization methods, which take the CEE into account, might be considered $[12,31,57,62,67]$ for use in monitor unit calculation programs, as well as for a reduction of the number of measurements needed to determine $S_{c}$ under all circumstances. In these parametrization methods, the equivalent squares are used as an auxilliary step in the calculation of $S_{c}$ of rectangular fields, see Appendix 8.7.2. 
8.

\subsection{Parametrization of scatter correction factors}

\subsubsection{Parametrization of phantom scatter correction factors}

$S_{p}$ data can be parametrized using the assumption that the phantom scatter at a specified depth for circular fields can be described as the sum of pencil beam contributions [66]. Each pencil is assumed to consist of three gaussian components, $a_{i} \cdot \exp \left(-b_{i} \cdot r^{2}\right)$ with $i=1,2$ and 3 . It was shown by van Gasteren et al. [66] that the following expression describes $S_{p}$ with sufficient accuracy as a function of the field radius $r_{d}$ (in $\mathrm{cm}$, taken at the depth of consideration, for example at $\left.d_{\text {ref }}\right)$ :

$$
S_{p}\left(r_{d}\right)=b_{1}+a_{1}\left\{\left(a_{2} / b_{2}\right)\left(1-\exp \left(-b_{2} \cdot r_{d}^{2}\right)\right)+\left(a_{3} / b_{3}\right)\left(1-\exp \left(-b_{3} \cdot r_{d}^{2}\right)\right)\right\}
$$

Parameters $a_{1}$ to $a_{3}$ and $b_{1}$ to $b_{3}$ (see table 8.4) were found with a least square method. For quality indices in between, values of $a_{1}$ to $b_{3}$ can be interpolated in first approximation, while the $S_{p}$ data of table 5.2 can be used for comparison.

Table 8.4 Quality index, QI, and parameters used in expression (8.7.1) for the calculation of $S_{p}\left(r_{d}\right)$ of circular fields with radius $r_{d}$ of four nominal photon beam energies [66].

\begin{tabular}{l|llll}
\hline parameter & ${ }^{60} \mathrm{Co}$ & $6 \mathrm{MV}$ & $10 \mathrm{MV}$ & $25 \mathrm{MV}$ \\
\hline Ql & 0.572 & 0.670 & 0.729 & 0.783 \\
& & & & \\
$\mathrm{a}_{1}$ & 0.5380 & 0.5519 & 0.5694 & 0.6991 \\
$\mathrm{~b}_{1}$ & 0.8092 & 0.8614 & 0.8792 & 0.8819 \\
$\mathrm{a}_{2}$ & 0.0180 & 0.0150 & 0.0140 & 0.0180 \\
$\mathrm{~b}_{2}$ & 0.0600 & 0.0650 & 0.0700 & 0.1200 \\
$\mathrm{a}_{3}$ & 0.0026 & 0.0013 & 0.0009 & 0.0006 \\
$\mathrm{~b}_{3}$ & 0.0070 & 0.0050 & 0.0045 & 0.0060 \\
\hline
\end{tabular}

The next step is to transform the phantom scatter data for circular fields to those for square fields. This can be done with a Clarkson integration, or by using the conversion method, which was previously described by Day and Aird [8] and discussed, in more detail, by Bjärngard and Siddon [4]:

and

$$
\mathrm{s} / 2 \mathrm{r}_{\mathrm{eq}}=0.891+0.00092 \mathrm{r}_{\mathrm{eq}}
$$

$$
2 r_{\text {eq }} / s=1.123-0.00067 \mathrm{~s}
$$

where $r_{\mathrm{eq}}$ is the radius of the (equivalent) circular, and $s$ the side of the (equivalent) square field, both in $\mathrm{cm}$ and defined at the surface, i.e. at SSD. For a proper application of equation $(8.7 .1), r_{\mathrm{eq}}$ has to be transformed to the radius 
at depth $d_{r e f}, r_{d}$, according to the divergence of the beam using a multiplication with (SSD $+d_{\text {ref }}$ )/SSD. The correspondence between the calculated and measured values of $S_{p}$ is better than $0.5 \%[66,70]$.

\subsubsection{Parametrization of collimator scatter correction factors}

The collimator exchange effect (CEE) necessitates that $S_{c}$ should be determined for a large number of fields $[15,33,58]$. For example, if the $X$ - and $Y$ - blocks are set to $3,4,5,6,8,10,12,15,20,25,30,35$ and $40 \mathrm{~cm}$, as is recommended in section $4, S_{c}$ has to be measured for 169 fields. Then, for each type of beam modifier, this number of measurements should be repeated, see section 3.1. A parametrization method for $S_{c}$ could considerably decrease the number of measurements. Two approaches have been used in the literature. One is based on analytical models, using suitable functions through the data $[4,55,57,62]$. The second approach makes use of more sophisticated, physical models $[2,3,15,35,40,58,67,74]$. The latter approach requires knowledge of properties of the accelerator, like the energy spectrum of the beam and/or the exact construction of the head of the treatment machine, that may not be available. However, analytical models are most valuable if these would be universally applicable. For any method the required accuracy is: $1 \%$ as the maximum difference between a measured and a calculated value of $S_{c} ; 0.5 \%$ as the root of the mean of the squares of differences (RMS) between calculated and measured $S_{c}$ values in a table. In a study of Jager et al. [31] several published methods were compared, using measured $S_{c}$ data from the treatment machines listed in table 5.1 of this report.

\section{An analytical model for $S_{c}$ of square fields}

Jager et al. [31] compared several published methods to fit $S_{c}$ data of square fields for a number of different linear accelerators. They found that $S_{c}$ could be described accurately by:

$$
S_{c}(X, X)=a_{1}+a_{2} \ln (X / 10)+a_{3} \ln ^{2}(X / 10)+a_{4} \ln ^{3}(X / 10)
$$

In this expression $a_{1}, a_{2}, a_{3}$ and $a_{4}$ are constants, of which the numerical values can be obtained with a least squares method on the difference between measured and fitted data. It was shown that by applying this polynomial, the RMS of the differences between measured and fitted $S_{c}$ values for square fields was less than $0.25 \%$ and the maximum deviation less than $0.5 \%$ for all treatment machines in the study.

To determine the parameters of equation (8.7.3) with the optimal accuracy, a minimum set of measured $S_{c}$ data of square fields must include the field sides 4 , $5,6,10,12,25,30$ and $40 \mathrm{~cm} \mathrm{[31].}$ 


\section{The equivalent square field size of rectangular fields and parametrisation of $S_{c}$}

In a further step, the equivalent field size for collimator scatter $v_{c}{ }^{e q}$ was used, defined as that equivalent square field that has the same $S_{c}$ value as the given rectangular field. A well-known concept to find $v_{\mathrm{eq}}$ of a rectangular field is the area-perimeter relation of Sterling et al. [55]: $v_{\mathrm{eq}}=2 X Y /(X+Y)$. Since this relation is symmetrical in $X$ and $Y$, it cannot include the CEE. A modification of this formulation has been presented by Vadash and Bjärngard [62]. Jager et al. [31] introduced the relation:

$$
v_{c, e q}(X, Y)=\left\{\left(a_{1} / X\right)+\left(a_{2} / X^{2}\right)+\left(b_{1} / Y\right)+\left(b_{2} / Y^{2}\right)\right\}^{-1}
$$

Sterling's relation [55] is found for the parameter values $a_{1}=b_{1}=0.5$ and $a_{2}=b_{2}=0$. Applying equation (8.7.4), the resulting equivalent square field size can now be used in the polynomial of $(8.7 .3)$ to obtain $S_{c}\left(v_{c, e q}\right)$. Parameter values of $a_{1}, \ldots b_{2}$ can be found from the least squares method.

For rectangular fields, the RMS of the differences between measured and fitted data for equation (8.7.4) was shown to be below $0.35 \%$ for a number of measured $S_{c}$ tables. The maximum deviations were less than $0.80 \%$ for all accelerators tested [31]. It was recommended to measure $S_{c}$ of the before mentioned set of square fields, and of twelve non-square fields: $4 \times 8,4 \times 30,4$ $\times 40,5 \times 40,8 \times 4,8 \times 40,30 \times 4,30 \times 40,40 \times 4,4 \times 5,40 \times 8$ and $40 \times$ 30 (all dimensions in $\mathrm{cm}$ ), and to apply equation (8.7.4) to fit the data.

The parameters that were found for the fitting methods described here are typically dependent on the construction of the head of the treatment machine. A correlation between the parameters and the quality index was not found [31].

\section{A physical model for the parametrization of $S_{c}$ of rectangular fields}

The influence of the CEE can be taken into account by using a correction factor on the $\mathrm{X}$ - or $\mathrm{Y}$-collimator field size $\left(\mathrm{C}_{\mathrm{fx}}\right.$ of $\mathrm{C}_{\mathrm{fy}}$, respectively). This correction factor $\mathrm{C}_{f}$ converts the two-dimensional, asymmetrical data set of $S_{c}$ values vs. the independent $X$ - and $Y$ - settings into a symmetrical data set (i.e. with $C_{f x} X X$ and $C_{f y} \times Y$ ). Values of $C_{f}$ can be found by using construction data of the treatment machine: from the ratio of distances from focus to upper, or lower collimator pairs, respectively. Another method is to use the measured 2-D data set of $S_{c}$ values and to apply $C_{f}$ iteratively as a multiplicative factor to the $X$ - or $Y$ - field side dimension, until a symmetrical table is obtained. Whether $C_{\mathrm{fx}}=1$ and $\mathrm{C}_{\mathrm{fy}} \neq$ 1 or vice versa, depends on the construction details of the machine, i.e. whether the $X$ or $Y$ setting is determined by the upper or lower pair of jaws.

The curves of $S_{c}$ data versus field size show, in its form, the same behaviour as the previously discussed $S_{p}$ curves. It is, then, not surprising to find that collimator scatter data can be described as a sum of pencil beam contributions in practically the same way as this was done for the phantom scatter data in the 
previous paragraph. This idea was developed by van Gasteren et al. [66]. The collimator scatter factor as a function of the radius, $r$, of a circular field can be found from:

$$
S_{c}(r)=b_{1}+a_{1}\left\{\left(a_{2} / b_{2}\right)\left(1-\exp \left(-b_{2} \cdot r^{2}\right)\right)+\left(a_{3} / b_{3}\right)\left(1-\exp \left(-b_{3} \cdot r^{2}\right)\right)\right\}
$$

where parameters $a_{1}$ to $a_{3}$ and $b_{1}$ to $b_{3}$ again can be found with a least square method by comparing measured and fitted data.

Having obtained a symmetrical set of data, a conventional table of equivalent field sizes can then be used to convert the rectangular field size $C_{f x} \times X, C_{f y} \times Y$, into an equivalent square, or rather an equivalent circular field $[8,9]$. The equivalent radius $r_{\text {eq }}$ can be used in the expression (8.7.5) to derive $S_{c}$. Thus, the set of measured square field sizes $(X, X)$ are converted to effective field sizes $\left(C_{f x} X, C_{f y} Y\right)$, which are equivalent to the effective circular fields with radii $r_{e q}$. The set of $S_{c}\left(r_{e q}\right)$ can be applied to calculate $S_{c}(X, Y)$ with the Clarkson integration method using the set $S_{c}\left(r_{e q}\right)$ for the $r_{e q}$ of each Clarkson sector.

The method was tested using beam data of several treatment machines, including Philips SL15 and SL20, ABB Dynaray20 and GE Saturne-43 linear accelerators, for photon beam qualities of 6 to $25 \mathrm{MV}$. Average deviations between measured and calculated data were in general below $0.5 \%$. The method was also tested for a number of beam data from asymmetrical collimator machines [67] and (a)symmetrical wedged fields [68].

In summary, if this method is applied, the following steps have to be taken:

1. Measure the $S_{c}(X, X)$ values from $4 \mathrm{~cm} \times 4 \mathrm{~cm}$ to $40 \mathrm{~cm} \times 40 \mathrm{~cm}$; measure also the $S_{c}(X, Y)$ of a number of extremely elongated fields: for example $(4,40),(5,40), \ldots$, and $(40,4),(40,5)$.

2. Replace the measured square field sizes $(X, X)_{\text {meas }}$ and the rectangular field sizes $(X, Y)_{\text {meas }}$ by $\left(X, C_{f y} X\right)$ and $\left(X, C_{f y} Y\right)$, respectively.

3. Determine for all fields $\left(X, C_{f y} X\right)$ the equivalent radius by geometric Clarkson integration, i.e. take for every degree the length of the radius to the contour $\left(X, C_{f y} X\right)$ and determine the average value of $r_{e q}$ of these radii. This results in a set of $S_{c}\left(r_{e q}\right)$ values. Thus, $S_{c}\left(r_{e q}\right)=S_{c}\left(X, C_{f y} X\right)=S_{c}(X, X)_{\text {meas }}$.

4. Calculate for all measured rectangular fields $(X, Y)_{\text {meas }}$ the corresponding $S_{c}\left(X, C_{f y} Y\right)$ by Clarkson integration of the field $\left(X, C_{f y} Y\right)$, using $S_{c}\left(r_{e q}\right)$. The Clarkson integration now determines for every degree the length of the radius $r$ to the field contour and interpolates the corresponding $S_{c}(r)$, using the set of $\mathrm{S}_{\mathrm{c}}\left(\mathrm{r}_{\mathrm{eq}}\right)$ values.

5. If the fit between measured and calculated $S_{c}$ values for the rectangular fields is not optimal, adjust the $Y$-collimator factor $C_{f y}$ and repeat the procedure, starting at step 2.

6. Note: depending on the machine type, it may be necessary to change $\left(X, C_{f y} Y\right)$ into $\left(C_{f x} X, Y\right)$, etc. in these procedures. 


\subsection{The narrow cylindrical beam-coaxial phantom (mini-phantom)}

In this report, the use of the mini-phantom is recommended for the measurement of the collimator scatter correction factor of the megavoltage photon beam. The results of the measurements should reflect as accurately as possible the change in the energy fluence due to primary photons coming from the treatment head at the reference depth with variation of the collimator setting. The collimator scatter correction factor is defined as the ratio of two measurements: one in an arbitrary field and one in the reference field. In each measurement, the resulting ionization is caused by the direct radiation from the head of the treatment machine and a small contribution of radiation scattered within the mini-phantom. Due to the material above the ionization chamber, a certain fraction of the direct radiation is absorbed. Because the amount of absorption in the material and the scattered radiation created within the phantom are directly proportional to the amount of direct radiation from the treatment head, both effects on the ionization in the detector cancel when we take the ratio of the two readings. The resulting collimator scatter correction factor is therefore almost independent of the construction details of the mini-phantom.

Two conditions must be met. First, the measurements have to be performed using the same mini-phantom, in which the depth of measurement is chosen large enough to eliminate the contaminating electrons in the beam. Second, the minimum field size in which measurements are performed, must be large enough to cover the phantom surface completely.

The depth of measurement is set to $10 \mathrm{~cm}$, according to the definitions and recommendations of this report. Experiments performed by the Task Group members and others have shown only minor deviations in the resulting $S_{c}$ values when the construction details were changed. The mini-phantom may be constructed with diverging side walls and square or circular cross sections. As construction materials solid PMMA or other water-equivalent materials may be used. A hollow PMMA phantom, to be filled with water, has also been used. The bottom side of the phantom may be provided with more backscattering material (i.e. the "length" of the mini-phantom).

The diameter of the mini-phantom also appears to be of minor importance. Several experiments have been performed with mini-phantoms of different sizes: $4 \mathrm{~cm}$ diameter down to $2 \mathrm{~cm}$. No significant differences have been observed, which is in agreement with the results of Li et al. [42]. However, care has to be taken in case of high energy beams in combination with a relatively small miniphantom. For these qualities, it is possible that the thin side walls can be penetrated by higher energy contaminating electrons from the treatment head, which influences the determination of $S_{c}$ [42]. For photon beams with a nominal beam quality exceeding $16 \mathrm{MV}$, it is recommended to have a minimum diameter of $3 \mathrm{~cm}$. Beams are supposed to have a relatively flat beam profile in air at the 
8.

position of the surface of the mini-phantom. For large fields this is always the case. However, in some situations, for example in small ${ }^{60} \mathrm{Co}$ fields, this may not be true, which may lead to small deviations. Therefore, a $3 \mathrm{~cm}$ diameter seems to be a good compromise between the different requirements.

The need for measurement of the collimator scatter correction factor for very small fields is another topic, e.g. encountered in the field of stereotactic radiosurgery techniques. For these situations, high $\mathrm{Z}$ build-up caps of different materials and different sizes are sometimes recommended. This is, however, not the subject of this report. More details can be found in the relevant literature (e.g., $[53,73])$.

Two drawings of the mini-phantom are shown in figures 8.4 and 8.5 . The upright position of the ionization chamber in figure 8.4 is preferred because the chamber is now symmetrically placed with respect to the beam axis. The influence of the stem effect on the readings has to be checked, but is in this situation of less importance than in the situation of figure 8.5 , where the chamber is placed horizontally in the beam. The effective point of measurement is determined by the dimensions and the construction of the ionization chamber and is dependent on the way in which it is irradiated, but it will be independent of the field size. Therefore, the depth is not a critical parameter in the determination of the collimator scatter correction factors and knowledge of the exact position of the effective point of measurement is not essential.

In case $S_{c p}$ measurements are performed in a large water-phantom, usually the same depth is chosen as applied for the calibration of the beam. Then, for absolute dose measurements, knowledge of the position of the effective point of measurement is necessary and a horizontal position of the ionization chamber is preferred.

When these considerations are taken into account, all ionization chambers available in the clinic may be used. No preference for a certain type of instrument exists. 
8.

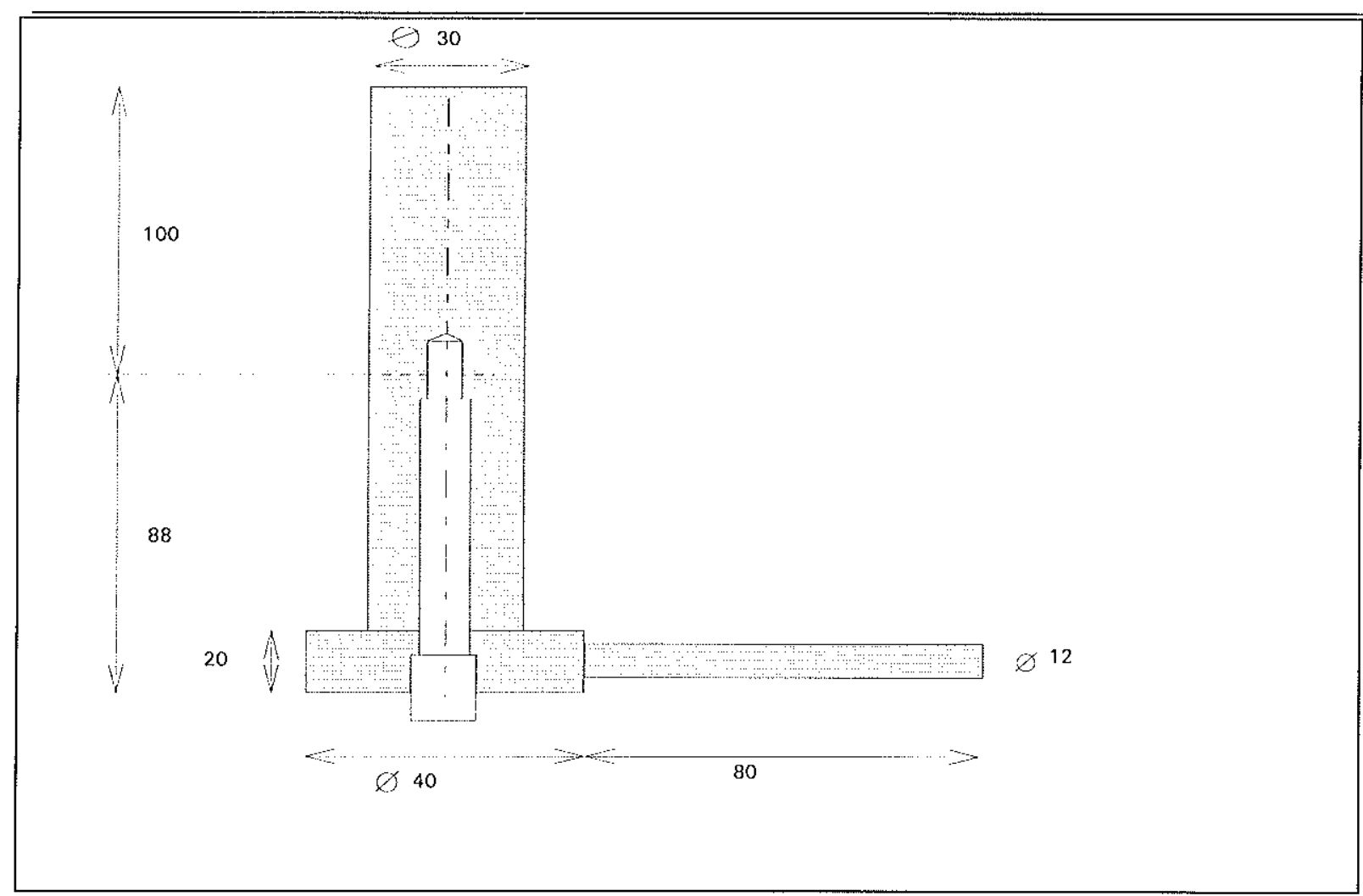

Figure 8.4 Construction drawing of the narrow cylindrical beam-coaxial (mini) phantom, in upright position. All dimensions in $\mathrm{mm}$.

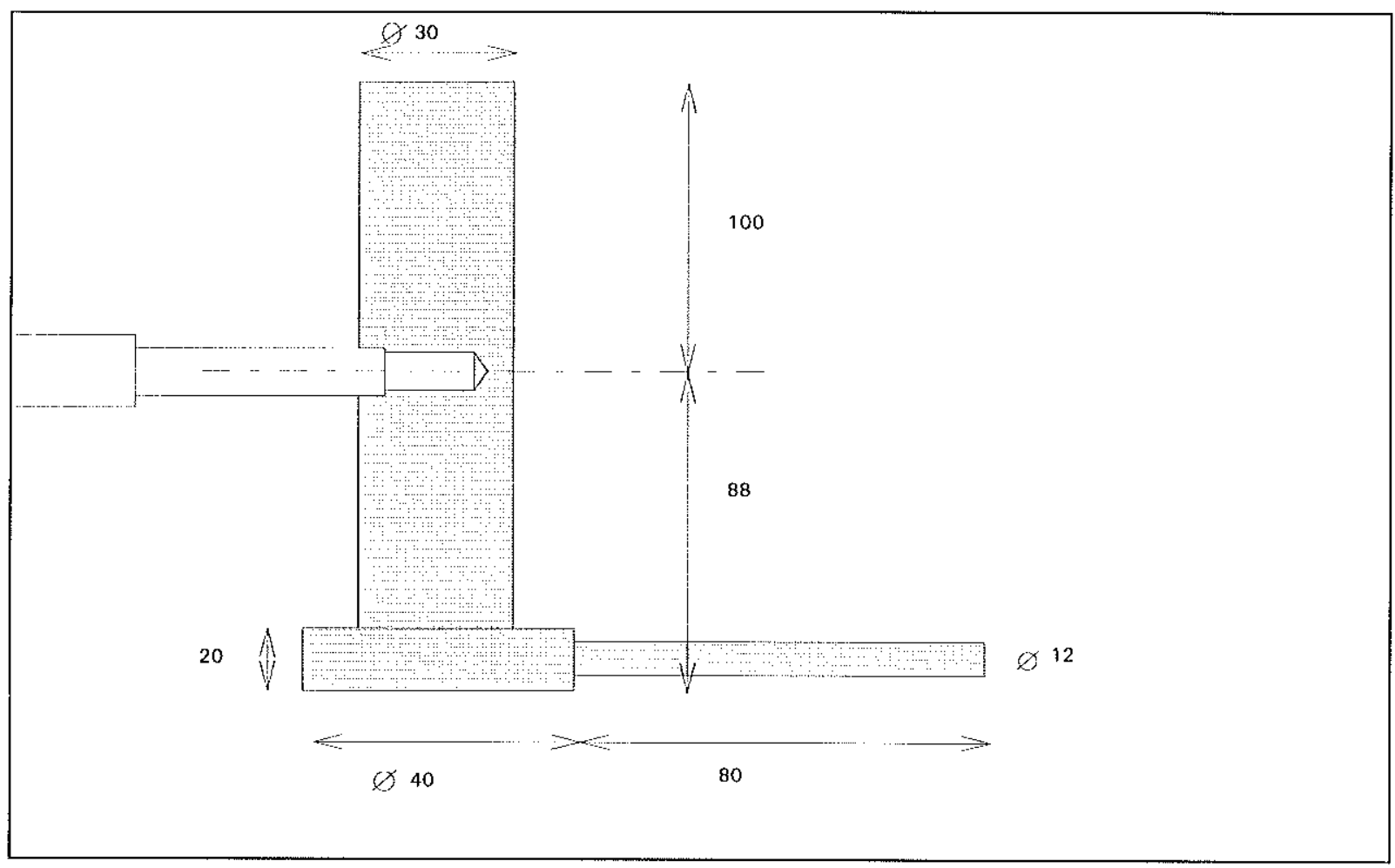

Figure 8.5 Construction drawing of the narrow cylindrical beam-coaxial (mini) phantom, in horizontal position. 


\section{References}

[1] Aget, H. Calcul de la dose sur l'axe dans des conditions autres que celles de référence, pour des faisceaux de photons. In: De la mesure à la dose absorbée en radiothérapie. pp 212-235, Clinique d'Oncologie et Radiothérapie, Hôpital Bretonneau C.H.U. Tours, France.

[2] Ahnesjö, A. Collimator scatter in photon therapy beams. Med. Phys, 22: 267-278, 1995.

[3] Ahnesjö, A., Knöös, T. and Montelius, A. Application of the convolution method for calculation of output factors for therapy photon beams. Med. Phys. 19: 295-301, 1992.

[4] Bjärngard, B.E. and Siddon, R.L. A note on equivalent circles, squares and rectangles. Med. Phys. 9: 258-260, 1982 .

[5] Bjärngard, B.E. and Vadash, P. Analysis of central-axis dose for high-energy $x$ rays. Med. Phys. 22: 1191-1195, 1995.

[6] Bjärngard, B.E., Zhu, T.C. and Ceberg, C. Tissue-phantom ratios from percentage depth doses. Med. Phys. 23: 629-634, 1996.

[7] Brahme, A., Chavaudra, J., Landberg, T., McCullough, E., Nüsslin, F., Rawlinson, A., Svensson, G. and Svensson, H. Accuracy requirements and quality assurance of external beam therapy with photons and electrons. Supplementum 1 to Acta Oncologica, 1988.

[8] Central Axis Depth Dose Data for Use in Radiotherapy. Brit. J. Radiol. Suppl. 17. Editors: D.K. Bewley, A.L. Bradshaw, D. Greene, J.L. Haybittle and L.F. Secretan. The British Institute of Radiology, 1983.

[9] Central Axis Depth Dose Data for Use in Radiotherapy: 1996. Brit. J. Radiol. Suppl. 25. Editors: E.G.A. Aird, J.E. Burns, M.J. Day, S. Duane, T.J. Jordan, A. Kacperek, S.C. Klevenhagen, R.M. Harrison, S.C. Lillicrap, A.L. McKenzie, W.G. Pitchford, J.E. Shaw and C.W. Smith. The British Institute of Radiology, 1996.

[10] CFMRI (Comité Français Mesure des Rayonnements lonisants). Recommendations pour la mesure de la dose absorbée en radiothérapie dans faisceaux de photons et d'électrons d'énergie comprise entre $1 \mathrm{MeV}$ et $50 \mathrm{MeV}$. Rapport 2, CFMRI

[11] Chaney, E.L., Cullip, T.J. and Gabriel, T.A. A Monte Carlo study of accelerator head scatter. Med. Phys. 21: 1383-1390, 1994.

[12] Chen, F.S. Applying a polynomial formula to photon beam output and equivalent square field. Med. Phys, 6: 464-469, 1990.

[13] Clarkson, J.R. A note on depth doses in fields of irregular shape. Brit. J. Radiol. 14: 265-268, 1941.

[14] Drouard, J., Rosenwald, J.C. and Simonian, M. Generation of primary and scatter tables for computer computations in high energy photon beams. Proc. 5th ESTRO meeting, Baden-Baden, 1986 (Abstract).

[15] Dunscombe, P.B. and Nieminen, J.M. On the field-size dependence of relative output from a linear accelerator. Med. Phys. 19: 1441-1444, 1992.

[16] Dutreix, A. When and how can we improve precision in radiotherapy? Radiother. Oncol. 2: 275292, 1984. 
9.

[17] Dutreix, A. The French dosimetry protocol. Radiother. Oncol, 4: 301-304, 1985.

[18] Dutreix, A., Bjärngard, B.E., Bridier, A., Mijnheer, B., Shaw, J.E. and Svensson, H. Monitor unit Calculation for high energy photon beams. ESTRO Booklet 3, Garant ed., Leuven, 1997.

[19] Goitein, M. Nonstandard deviations. Med. Phys, 10: 709-711, 1983.

[20] Heukelom, S., Lanson, J.H. and Mijnheer, B.J. Wedge factor constituents of high energy photon beams: field size and depth dependence. Radiother. Oncol. 30: 66-73, 1994.

[21] Heukelom, S., Lanson, J.H. and Mijnheer, B.J. Quality assurance of the simultaneous boost technique for prostatic cancer: dosimetric aspects. Radiother. Oncol. 30: 74-82, 1994.

[22] Heukelom, S., Lanson, J.H. and Mijnheer, B.J. Wedge factor constituents of high energy photon beams: head and phantom scatter dose components. Radiother. Oncol. 32: 73-83, 1994.

[23] Heukelom, S., Lanson, J.H. and Mijnheer, B.J. Differences in wedge factor determination in air using a PMMA mini-phantom or a brass build-up cap. Med. Phys. 24: 1986-1991, 1997.

[24] Higgins, P.D., Sohn, W.H., Sibata, C.H. and McCarthy, W.A. Scatter factor corrections for elongated fields. Med. Phys, 16: 800-802, 1989.

[25] Holt, J.G., Laughlin, J.S. and Moroney, J.P. The extension of the concept of tissue-air-ratios (TAR) to high energy $X$-ray beams. Radiol. 96: 437-446, 1970.

[26] Huang, P., Chin, L.M. and Bjärngard, B.E. Scattered photons produced by beam-modifying filters. Med. Phys. 13: 57-63, 1986.

127] Huang, P., Chu, J. and Bjärngard, B.E. The effect of collimator backscatter on photon output of linear accelerators. Med. Phys. 14: 268-269, 1987.

[28] [AEA (International Atomic Energy Agency). Absorbed dose determination in photon and electron beams. Technical Report 277, IAEA, Vienna, 1987.

[29] ICRU (International Commission on Radiation Units and Measurements). Determination of absorbed dose in a patient irradiated by beams of $X$ or gamma rays in radiotherapy procedures. Report 24. ICRU Publications, Washington, D,C.,1976.

[30] Islam, M.K. and Van Dyk, J. Effects of scatter generated by beam-modifying absorbers in megavoltage photon beams. Med. Phys.22: 2075-2081, 1995.

[31] Jager, H.N., Heukelom, S., van Kleffens, H.J., van Gasteren, J.J.M., van der Laarse, R., Mijnheer, B.J., Venselaar, J.L.M. and Westermann, C.F. Comparison of parametrization methods of the collimator scatter correction factor for open rectangular fields of 6-25 MV photon beams. Radiother. Oncol, 45: 235-243, 1997.

[32] Johns, H.E., Bruce, W.R. and Reid, W.D. The dependence of depth dose on focal skin distance. Brit. J. Radiol. 31: 254-260, 1958.

[33] Kase, K.R. and Svensson, G.K. Head scatter data for several linear accelerators (4-18 MV). Med. Phys, 13: 530-532, 1986.

[34] Khan, F.M., Sewchand, W., Lee, J. and Williamson, J.F. Revision of tissue-maximum ratio and scatter maximum ratio concepts for cobalt-60 and higher energy X-ray beams. Med. Phys. 7: 230-237, 1980.

[35] Kim, S., Zhu, T.C. and Palta, J.R. An equivalent square field formula for determining head scatter factors of rectangular fields. Med. Phys. 24: 1770-1774, 1997.

[36] Krithivas, G. and Rao, S.N. A study of the characteristics of radiation contaminants within a clinically useful photon beam. Med. Phys. 12: 764-768, 1985. 
9.

[37] Krithivas, G, and Rao, S.N. Dosimetry of $24 \mathrm{MV}$ x-rays from a linear accelerator. Med. Phys. 14: 274-281, 1987.

[38] Kubo, H. Telescopic measurements of backscattered radiation from secondary collimator jaws to a beam monitor chamber using a pair of slits. Med. Phys. 16: 295-298, 1989.

[39] Kubo, H. and Lo, K.K. Measurement of backscattered radiation from Therac-20 collimator and trimmer jaws into the beam monitor chamber. Med. Phys. 16: 292-294, 1989.

[40] Lam, K.L., Muthuswamy, M.S. and Ten Haken, R.K. Flattening-filter-based empirical methods to parametrize the head scatter factor. Med. Phys, 23: 343-352, 1997.

[41] Lam, K.L, and Ten Haken, R.K. In phantom determination of collimator scatter factor, Med. Phys. 23: 1207-1212, 1996.

[42] Li, X.A., Soubra, M., Szanto, J. and Gerig, L.H. Lateral electron equilibrium and electron contamination in measurements of head-scatter factors using miniphantoms and brass caps. Med.Phys. 22: 1167-1170, 1995.

[43] Ling, C.C., Schell, M.C. and Rustgi, S.N. Magnetic analysis of the radiation components of a 10 MV Photon beam. Med. Phys. 9: 20-26, 1982.

[44] Luxton, G, and Astrahan, M.A. Characteristics of the high-energy photon beam of a $25 \mathrm{MV}$ accelerator. Med. Phys. 15: 82-87, 1988.

[45] Luxton, G, and Astrahan, M.A. Output factor constituents of a high-energy photon beam. Med. Phys. 15: 88-91, 1988.

[46] Meli, J.A. Output factors and dose calculations for blocked x-ray fields. Med. Phys. 13: 405-408, 1986.

[47] Mijnheer, B.J., Aalbers, A.H.L., Visser, A.G. and Wittkämper, F.W. Consistency and simplicity in the determination of absorbed dose to water in high-energy photon beams: $A$ new code of practice. Radiother. Oncol. 7: 371-384, 1986.

[48] Mijnheer, B.J., Battermann, J.J. and Wambersie, A. What degree of accuracy is required and can be achieved in photon and neutron therapy? Radiother. Oncol. 8: 237-252, 1987.

[49] Mijnheer, B.J., Wittkämper, F.W., Aalbers, A.H. and van Dijk, E. Experimental verification of the air kerma to absorbed dose conversion factor $C_{w, u}$. Radiother. Oncol. 8: 49-56, 1987.

[50] Patomäki, L.K. The equivalent field principle and its use in beam therapy dose calculations. Brit. J. Radiol. 41: 381-383, 1968.

[51] Patterson, M.S, and Shragge, P.C. Characteristics of an 18 MV photon beam from a Therac-20 medical linear accelerator. Med. Phys. 10:312-318, 1981.

[52] Podgorsak, M.B., Kubsad, S.S. and Paliwal, B.R. Dosimetry of large wedged high-energy photon beams. Med. Phys. 20: 369-373, 1993.

[53] Rice, R.K., Hansen, J.L., Svensson, G.K. and Siddon, R.L. Measurements of dose distributions in small beams of 6 MV X-rays. Phys. Med. Biol. 32: 1087-1099, 1987.

[54] Spicka, J., Herron, D. and Orton, C. Separating output factor in collimator and phantom scatter factor for megavoltage photon calculations. Med. Dos. 13: 23-24, 1988.

[55] Sterling, T.D., Perry, H. and Katz, L. Automation of radiation treatment planning. Brit. J. Radiol. 37: 544-550, 1964.

[56] Storchi, P. and van Gasteren, J.J.M. A table of phantom scatter factors of photon beams as a function of the quality index and field size. Phys. Med. Biol. 41: 563-571, 1996. 
9.

[57] Szymczyk, W., Goraczko, A. and Lesiak, J. Prediction of Saturne II+ $10 \mathrm{MV}$ and $23 \mathrm{MV}$ photon beam output factors. Int. J. Radiat. Oncol. Biol. Phys. 21: 789-793, 1991.

[58] Tatcher, M. and Bjärngard, B.E. Head-scatter factors in rectangular photon fields. Med. Phys. 20: 205-206, 1993.

[59] Tatcher, M. and Bjärngard, B.E. Equivalent squares of irregular photon fields. Med Phys. 20: 1229-1232, 1993.

[60] Tatcher, M. and Bjärngard, B.E. Head-scatter factors in blocked photon fields. Radiother. Oncol. 33: 64-67, 1994.

[61] Thomas, S.J. The variation of wedge factors with field size on a linear accelerator. Brit. J. Radiol. 63: 355-356, 1990.

[62] Vadash, P. and Bjärngard, B.E. An equivalent-square formula for head-scatter factors. Med Phys. 20: 733-734, 1993.

[63] van Dam, J., Bridier, A., Lasselin, C., Blanckaert, N. and Dutreix, A. Influence of shielding blocks on the output of photon beams as a function of energy and type of treatment unit. Radiother. Oncol. 24:55-59, 1992.

[64] van Gasteren, J.J.M., Heukelom, S., van Kleffens, H.J., van der Laarse, R., Venselaar, J.L.M. and Westermann, C.F. The determination of phantom and collimator scatter components of the output of megavoltage photon beams: measurement of the collimator scatter part with a beamcoaxial narrow cylindrical phantom. Radiother. Oncol. 20: 250-257, 1991.

[65] van Gasteren, J.J.M., Heukelom, S., van Kleffens, H.J., van der Laarse, R., van der Linden, P.M., Venselaar, J.L.M. and Westermann, C.F. The phantom scatter correction factor $\mathrm{Sp}$ of the photon beam output factor Scp as a function of the beam energy in the range from 2 to $25 \mathrm{MV}$. Proc. XIth ICCR (Intern. Conf. on the Use of Computers in Radiation Therapy), 1994, Manchester, U.K. 284-285.

[66] van Gasteren, J.J.M., Heukelom, S., Jager, H.N., van Kleffens, H.J., van der Laarse, R., Venselaar, J.L.M. and Westermann, C.F. A 3-gaussian fit of phantom scatter correction data. Accepted Phys. Med. Biol., 1997.

[67] van Gasteren, J.J.M., Heukelom, S., Jager, H.N., van Kleffens, H.J., van der Laarse, R., Venselaar, J.L.M. and Westermann, C.F. On the parameterization of headscatter correction factors $S_{c}$ of rectangular fields of photon beams. 1. Symmetrical and asymmetrical open beams. Submitted in revised form to Phys. Med. Biol., 1998.

[68] van Gasteren, J.J.M., Heukelom, S., Jager, H.N., van Kleffens, H.J., van der Laarse, R., Venselaar, J.L.M. and Westermann, C.F. On the parameterization of headscatter correction factors $S_{c}$ of rectangular fields of photon beams. II. (a)symmetrical wedged beams. Submitted in revised form to Phys. Med. Biol., 1998.

[69] van Kleffens, H.J., van Gasteren, J.J.M., Heukelom, S., Jager, H.N., van der Laarse, R., Mijnheer, B.J., Venselaar, J.L.M. and Westermann, C.F. Dependence of the tray transmission factor on the collimator setting in high-energy photon beams. Proc. XII ICCR (Intern. Conf. on the Use of Computers in Radiation Therapy), Salt Lake City, USA, 1997 (Abstract).

[70] Venselaar, J.L.M., Heukelom S., Jager H.N., Mijnheer B.J., van Gasteren, J.J.M., van Kleffens H.J., van der Laarse R. and Westermann C.F. Is there a need for a revised table of equivalent square fields for the determination of phantom scatter correction factors? Phys, Med. Biol. 42: 2369-2381, 1997.

[71] Venselaar, J.L.M., van Gasteren, J.J.M., Heukelom S., Jager H.N., Mijnheer B.J., van der Laarse R., van Kleffens, H.J. and Westermann C.F. A consistent formalism for the application of phantom and collimator scatter factors. Phys. Med. Biol. Submitted for publication, 1998. 
9.

[72] Venselaar, J.L.M., Heukelom S., Jager H.N., Mijnheer B.J., van der Laarse R., van Gasteren, J.J.M., van Kleffens, H.J. and Westermann C.F. The relation between scatter correction factors defined at dose maximum and at a reference depth of $10 \mathrm{~cm}$. Phys. Med. Biol. Submitted for publication, 1998.

[73] Weber, L., Nilsson, P. and Ahnesjö, A. Build-up cap materials for measurement of photon headscatter factors. Phys. Med. Biol. 42: 1875-1886, 1997.

[74] YU, M.K. and Sloboda, R. Analytical representation of head scatter factors for shaped photon beams using a two-component $x$-ray source model. Med. Phys. 22: 2045-2055, 1995.

[75] Zhu, T.C. and Bjärngard, B.E. Scattered photons from wedges in high-energy x-ray beams. Med. Phys. 22: 1339-1342, 1995. 


\section{Publications of the Netherlands Commission on Radiation Dosimetry}

Radiation dosimetry activities in the Netherlands.

Inventory compiled under the auspices of the Netherlands Commission for Radiation

n.a.

Dosimetry.

NCS Report 1, July 1986.

Code of practice for the dosimetry of high-energy photon beams.

NCS Report 2, December 1986.

$f 20$,-

Proceedings of the Symposium on Thermoluminescence Dosimetry.

NCS Report 3, October 1988.

$f 20,-$

Aanbevelingen voor dosimetrie en kwaliteitscontrole van radioactieve bronnen bij brachytherapie

NCS Report 4, Februari 1989 (in Dutch).

Recommendations for dosimetry and quality control of radioactive sources used in

brachytherapy.

Synopsis (in English) of NCS Report 4, February 1991.

Code of practice for the dosimetry of high-energy electron beams.

NCS Report 5, December 1989.

Dosimetric aspects of Mammography.

NCS Report 6, March 1993.

$f 25$,

Recommendations for the calibration of Iridium-192 high dose rate sources.

NCS Report 7, December 1994.

$f 25,-$

Kwaliteitscontrole van Medische Lineaire Versnellers, methoden voor kwaliteitscontrole, wenselijke toleranties en frequenties.

$f 25,-$

NCS Report 8, December 1995 (in Dutch).

Quality Control of Medical Linear Accelerators, current practice and minimum requirements. NCS Report 9, August 1996.

Dosimetry of low and medium energy $x$-rays, a code of practice for use in radiotherapy and radiobiology.

$f 25,-$ NCS Report 10, July 1997.

Quality Control of Simulators and CT scanners and some basic QC methods for Treatment Planning Systems, current practice and minimum requirements.

NCS Report 11, September 1997.

Determination and use of scatter correction factors of megavoltage photon beams, measurement and use of collimator and phantom scatter correction factors of arbitrarily shaped fields with a symmetrical collimator setting.

NCS Report 12, March 1998.

From within The Netherlands, reports can be ordered by paying the amount into ABN-AMRO account $\mathrm{nr}, 51.70 .64 .332$ to the credit of "Nederlandse Commissie voor Stralingsdosimetrie", stating the report number(s). Please add $f 5,-$ for postage and packing. 
From outside The Netherlands, please complete the order form below.

\section{ORDER FORM}

Please send this completed order form to:

NCS secretary, P.O.Box 654, 2600 AR DELFT, The Netherlands

OTY REPORT NUMBER AND TITLE

Add postage and packing (Europe $f 10,-$, outside Europe $f 20,-)$

Payment by: $\quad \square \quad$ Cheque/postal order made payable to Netherlands Commission on Radiation Dosimetry (add $f 15$,- for banking costs)

$\square \quad$ Please charge my Eurocard/Mastercard

Credit card No.

Expiry date

Name:

Address:

Date:

Signature: 